2019-04-08

\title{
Instabilities of Nanofluid Flow Displacements in Porous Media
}

\author{
Dastvareh, Behnam
}

Dastvareh, B. (2019). Instabilities of nanofluid flow displacements in porous media (Unpublished doctoral thesis). University of Calgary, Calgary, AB.

http://hdl.handle.net/1880/110149

Downloaded from PRISM Repository, University of Calgary 


\title{
UNIVERSITY OF CALGARY
}

Instabilities of Nanofluid Flow Displacements in Porous Media

by

Behnam Dastvareh

\begin{abstract}
A THESIS
SUBMITTED TO THE FACULTY OF GRADUATE STUDIES

IN PARTIAL FULFILLMENT OF THE REQUIREMENTS FOR THE

DEGREE OF DOCTOR OF PHILOSOPHY
\end{abstract}

GRADUATE PROGRAM IN CHEMICAL AND PETROLEUM ENGINEERING

CALGARY, ALBERTA

APRIL, 2019

(C) Behnam Dastvareh 2019 


\begin{abstract}
The interface of two approaching fluids in porous media becomes unstable at strong enough flow rates when the viscosity of the displacing fluid is less than that of the displaced one. This phenomenon is studied to address the effect of nanoparticles (NPs) dispersed in the displacing fluid assumed fully miscible with the displaced one. The problem is first studied under isothermal conditions. The effects of the NP-induced additional properties such as the viscosity of the nanofluid, the Brownian diffusivity and the NP deposition are addressed on both the flow instability and the flow configuration. It was found that NPs attenuate the instability of an initially unstable flow, but this effect is mitigated in the presence of NP deposition. Moreover, the Brownian diffusivity was found to have a destabilizing effect, but it cannot make an initially stable system unstable. The study is then extended to include the thermal effects. This leads to the emergence of a new NP transport phenomenon known as thermophoresis in which NPs migrate in opposite direction of the temperature gradient. This effect is addressed in connection with other properties. Specifically, depending on whether a hot fluid is displacing a cold one or vice versa, the competition between the two transport mechanics, Brownian motion and thermophoresis, is found to lead to different trends in terms of the flow configuration and instability. Next, the catalytic roles of NPs on the flow and instability are investigated for approaching reactive fluids. The study is conducted under both isothermal and non-isothermal conditions resulting from the heat of the reaction. A new set of conditions is introduced to predict the instability of the isothermal case based on the species mobility ratios, which then leads to six different flow configurations. Finally, the coupled effects of the heat of reaction and thermophoresis on the flow configuration and the amount of chemical products are addressed.
\end{abstract}




\section{Acknowledgements}

I first thank my supervisor, Dr. J. Azaiez for his support during this journey. I learned a lot both from his knowledge and personality. Then I thank the known and unknowns reviewers who made this work stronger by giving their generous advice. I also thank the University of Calgary for its Eyes High Scholarship program and the Natural Sciences and Engineering Research Council of Canada (NSERC) for their financial support. Finally, I acknowledge the use of computing resources of the West-Grid clusters. 


\section{Table of Contents}

$\begin{array}{ll}\text { Abstract } & \text { ii }\end{array}$

$\begin{array}{ll}\text { Acknowledgements } & \text { iii }\end{array}$

Table of Contents $\quad$ iv

List of Figures and Illustrations vi

List of Tables $\quad$ ix

List of Symbols, Abbreviations and Nomenclature $\quad \mathrm{x}$

1 Introduction $\quad 1$

2 Background $\quad 4$

2.1 Viscous fingering phenomenon . . . . . . . . . . . . . . . . . 4

2.1.1 Diffusion/Dispersion in miscible viscous fingering . . . . . . . . . . . 9

2.1.2 Permeability heterogeneity in miscible viscous fingering . . . . . . . . 12

2.2 Thermo-viscous fingering phenomenon . . . . . . . . . . . . 16

2.3 Viscous fingering of reactive fronts . . . . . . . . . . . . . . . . . . 19

2.4 Controlling viscous fingering with nanoparticles . . . . . . . . . . . . 23

3 Dynamics of Isothermic Nano-flow Displacements in Porous Media 26

3.1 Mathematical model . . . . . . . . . . . . . . . . . . . . 26

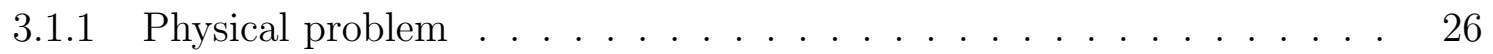

3.1 .2 Governing equations . . . . . . . . . . . . . . . . . . 27

3.2 Linear stability analysis . . . . . . . . . . . . . . . . . . . . . . . . . . . . . . . . . . . . . .

3.2.1 Problem formulation . . . . . . . . . . . . . . . . . . 30

3.2 .2 Analytical solution ... . . . . . . . . . . . . . . . . . . . . 33

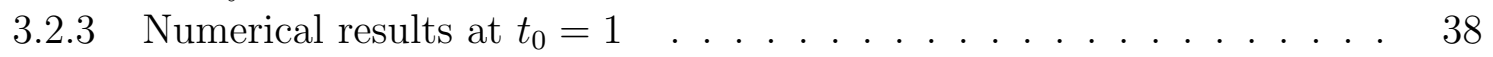

3.3 Non-linear simulations . . . . . . . . . . . . . . . . . . . . . . . . . . 42

3.3.1 Numerical technique . . . . . . . . . . . . . . . . . . . . . 42

3.3.2 Results and discussion ......................... 45 
4 Thermophoretic Effects on Instabilities of Nanoflows in Porous Media 53

4.1 Physical problem . . . . . . . . . . . . . . . . . . . 54

4.2 Problem formulation . . . . . . . . . . . . . . . . . . . 55

4.3 Numerical method . . . . . . . . . . . . . . . . . . . . . . . 61

4.4 Results and discusion . . . . . . . . . . . . . . . . . . . . . . . . . 64

4.4.1 Brownian diffusion . . . . . . . . . . . . . . . . . 65

4.4 .2 Thermophoresis . . . . . . . . . . . . . . . . . . . 71

4.4.3 Thermophoretic effects in stable/intinsically stable systems . . . . . . 82

4.4.4 Thermophoresis and Brownian diffusion in the energy equation . . . . 83

5 Dynamics of Nano-Catalytic Reactive Flows in Porous Media 85

5.1 Physical problem . . . . . . . . . . . . . . . . . . . 86

5.2 Problem formulation . . . . . . . . . . . . . . . . . . . . . . 87

5.3 Numerical methods . . . . . . . . . . . . . . . . . . . . . . . . . . . . . . . . . . 89

5.4 Results and discussion . . . . . . . . . . . . . . . . . . . . . 91

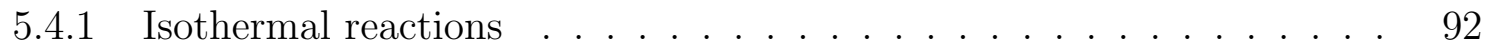

5.4 .2 Non-isothermal reactions . . . . . . . . . . . . . . . . . . . . . 98

$\begin{array}{llr}6 & \text { Conclusion } & 107\end{array}$

$\begin{array}{lr}\text { Bibliography } & 113\end{array}$

$\begin{array}{ll}\text { A Derivation of the Linear Disturbed Equations } & 125\end{array}$

B Derivation of the Characteristic Equations for Sharp Front 127

C Stability Criteria and Characteristic Equation for Intrinsically Stable Displacement

D Solving the non-linear equations with pseudo-spectral method 


\section{List of Figures and Illustrations}

$2.1\left(R_{s}, R_{f}\right)$ plain of six different base state viscosity distributions in double diffusive miscible displacements reported by Mishra et al. [4]. $R_{s}$ and $R_{f}$ are the $\log$ viscosity ratios of the slow and fast diffusing components while $\delta=\frac{D_{f}}{D_{s}}$. $L_{n}(n=1,2,3)$ denotes lines of $R_{f}=-\delta^{\frac{n}{2}} R_{s} \ldots \ldots \ldots \ldots$

2.2 The base state viscosity distributions in double diffusive-double convective miscible displacements reported by Azaiez and Sajjadi [43]. $\beta_{1}$ and $\beta_{2}$ are the solutal and thermal $\log$ viscosity ratio respectively, $\delta$ is the symbol used to represent Le number and $\lambda$ is the thermal lag coefficient.

2.3 Large time viscosity profiles of reactive systems with identical component diffusivities undergoing $A+B \rightarrow C$ reaction in $\left(R_{b}, R_{c}\right)$ plane. [53] . . . . 23

3.1 Schematic view of the medium . . . . . . . . . . . . . . . .

3.2 Characteristic curve for $R_{a}=R_{b}, \delta_{b}=1, \delta_{n}=1, D a_{d e p}=0.01$ and different $R_{n}$. Lines represent the results of Eq. 3.24 and symbols represent the

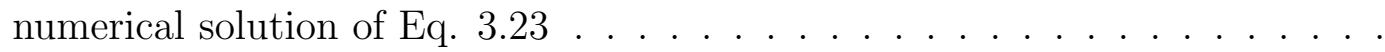

3.3 Characteristic curves at different nanofluid viscosity, $\delta_{n}=1, D a_{d e p}=0.01$, (a) $\delta_{b}=1$, (b) $\delta_{b}=2 \ldots \ldots \ldots \ldots$

3.4 Variation of the base state viscosity along the domain at higher $\delta_{b}=2, \delta_{n}=1$,

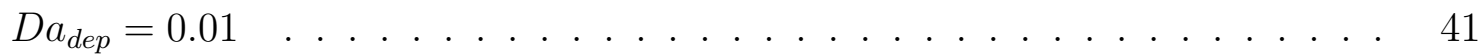

3.5 Effect of the NP diffusion, $R_{n}=1, D a_{\text {dep }}=0.01$ (a) $\delta_{b}=1$, (b) $\delta_{b}=2 \ldots . .41$

3.6 Effect of the diffusion of both displaced fluid and NPs when $R_{b}<R_{a}$ and $R_{n}>0, R_{a}=2, R_{b}=1, R_{n}=1, D a_{d e p}=0.01$, (a) $\delta_{n}=1$, (b) $\delta_{b}=0.5 \ldots$.

3.7 Iso-contours of $C_{a}\left(0.1 \leq C_{a} \leq 0.6\right)$ from the present study (left) and Singh

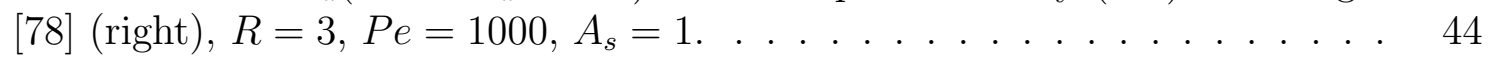

3.8 Contours of $C_{a}$ at different NP deposition rate, $R_{n}=1, \delta_{b}=1, \delta_{n}=1$. . . 45

3.9 Mixing length over time for different deposition rates, $R_{n}=1, \delta_{b}=1, \delta_{n}=1 \quad 46$

3.10 Contours of $C_{a}$ and $C_{n}$ at three NP deposition rates at $t=500, R_{n}=1$, $\delta_{b}=1, \delta_{n}=1$. It is seen that at $D a_{d e p}=0.5$ the NPs are completely removed from the system. . . . . . . . . . . . . . . . . .

3.11 Contours of $C_{a}$ representing the role of deposition rate in changing the stability of the system, $R_{n}=5, \delta_{b}=1, \delta_{n}=1$

3.12 (a) variation of the base state concentration along the channel, (b) variation of the base state viscosity along the channel, $R_{n}=5, \delta_{b}=1, \delta_{n}=1, D a_{d e p}=0.0148$

3.13 Contours of $C_{a}$ for different nanofluid viscosity ratio $\delta_{b}=1, \delta_{n}=1, D a_{\text {dep }}=0.0149$ 
3.14 (a) Mixing length, (b) breakthrough time, at different nanofluid viscosity ratios, $\delta_{b}=1, \delta_{n}=1, D a_{d e p}=0.01 \ldots \ldots \ldots$

3.15 Contours of $C_{a}$ for different NP diffusion rate $R_{n}=1, \delta_{b}=1, D a_{d e p}=0.01$.

3.16 (a) Variation of mixing length with time (b) transversely averaged concentration along the channel, $R_{n}=1, \delta_{b}=1, D a_{d e p}=0.01 \ldots \ldots . . . .$.

3.17 Contours of $C_{a}$ for different diffusion rate of displaced fluid $R_{n}=1, \delta_{n}=1$,

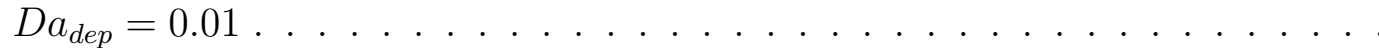

3.18 Finger configuration when $\delta_{b}=1.5$ in both (a) particle-free and (b) particleincluded $\delta_{n}=1, D a_{\text {dep }}=0.01$ systems . . . . . . . . . . . . .

4.1 Schematic view of the flow geometry. . . . . . . . . . . . . .

4.2 (a) Instability characteristics curve from LSA (b) iso-contour of $C_{b}=0.5$ at early times from NLS. $R_{b}=3, R_{\theta}=1, R_{n}=1, \lambda=0.8, \delta_{n}=1, \delta_{T}=3$, $L e=3, \epsilon=0.01, D a_{d e p}=0, r=0.5, t_{0}=1, P e=1024, A_{s}=2 . \ldots . .$.

4.3 Contours of $C_{b}$ for two values of Brownian diffusivity in both HDC and CDH systems. . . . . . . . . . . . . . . . . .

4.4 Variation of the Mixing Length with time for different Brownian diffusivities, (a) HDC system and (b) CDH system.

4.5 Variation of the transversely averaged NP concentration along with contours of $C_{n}$ for both HDC and CDH systems, (a) $\delta_{n}=0.1$ and (b) $\delta_{n}=1.0 \ldots$. .

4.6 A closer view of the contour of $C_{n}$ illustrated in Fig. 4.5 b. The accumulation of NPs in the region between the backward growing fingers is the result of the counter-spinning dipoles $\left(\delta_{n}=1\right) \ldots \ldots \ldots \ldots$

4.7 Viscosity contours for two values of Brownian diffusivity in the CDH system, (a) $\delta_{n}=0.1$ and $\left(\right.$ b) $\delta_{n}=1.0 \ldots \ldots \ldots \ldots$

4.8 A closer view of the viscosity contour depicted in Fig. 4.7a along with the streamlines. . . . . . . . . . . . . . . . . . . .

4.9 Variation of the transversely averaged NP concentration with insets of NP concentration contours at different temperature ratios for (a) HDC system

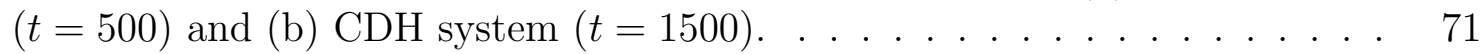

4.10 Contours of $C_{b}$ for different thermophoretic diffusivities in the HDC system. 72

4.11 Variation with time of the Mixing length for different thermophoretic diffusivities in the HDC system. . . . . . . . . . . . . . . . . . 72

4.12 Contours of $C_{n}$ for different thermophoretic diffusivities in the HDC system. 73

4.13 Contours of $C_{b}$ for different thermophoretic diffusivities in the CDH system. 74

4.14 Contours of $C_{n}$ and viscosity for different thermophoretic diffusivity in the $\mathrm{CDH}$ system. . . . . . . . . . . . . . . . . . 75

4.15 Contours of concentrations and viscosity in the CDH system for $\delta_{T}=20$. . $\quad 75$

4.16 Variation with time of the Mixing Length for different thermophoretic diffusivities in the CDH system.

4.17 The closer view of the viscosity contour of Fig. 4.15 along with the streamlines, $\delta_{T}=20 \ldots \ldots \ldots \ldots \ldots \ldots \ldots \ldots \ldots \ldots \ldots \ldots \ldots \ldots \ldots \ldots \ldots$

4.18 Base state NP concentration and a scaled variation of $\frac{\partial \bar{\theta}}{\partial x}$ and $\frac{\partial^{2} \ln \bar{\theta}}{\partial x^{2}}$ along the 77 channel in the HDC system at $t=500$. 
4.19 A typical variation of $\delta_{T_{c r}}$ with $\delta_{n}$ in the HDC system at $t=500$ and other default parameters. . . . . . . . . . . . . . . . .

4.20 Base state NP concentration and a scaled variation of $\frac{\partial \bar{\theta}}{\partial x}$ and $\frac{\partial^{2} \ln \bar{\theta}}{\partial x^{2}}$ along the channel at $\mathrm{t}=1500$ in the CDH system. . . . . . . . . . . . . . 80

4.21 Contours of $C_{b}$ in the HDC system with $R_{n}=5 \ldots \ldots 2$

4.22 Contours of $C_{b}$ in the HDC system for $R_{b}=-1$ in (a) the absence, (b) the presence of NPs. Note that $R_{n}$ and $\epsilon$ are set to zero to generate the case without NPs. . . . . . . . . . . . . . . . . . . . . .

5.1 Schematic view of the medium . . . . . . . . . . . . . . . .

5.2 The contours of $C_{c}$ in the isothermal NP-free reactive system derived from the present study (left) and Hejazi and Azaiez [54] (right), $R_{b}=3, R_{c}=5$, $D a=0.5, P e=1024, A_{s}=2 \ldots \ldots \ldots \ldots$

5.3 One dimensional Log-viscosity variation with respect to $\eta=\frac{x}{2 \sqrt{t}}$ at asymptotically large times $(t \rightarrow \infty)$ along with insets of corresponding contours of $C_{c}$ derived from NLS. The contours are represented at $t=1000$ for intrinsically unstable and $t=1300$ for intrinsically stable systems. . . . . . . . . . .

5.4 Variation of the normalized first moment of the transversely averaged product concentration with the inset of contours of $C_{c}$. Dashed lines represent those

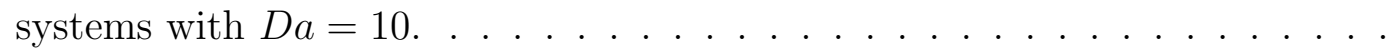

5.5 The effect of $R_{c}$ on the total amount of chemical products in the NP-laden isothermal reactive systems in the $\log -\log$ scale. . . . . . . . . . . . . .

5.6 The variation of $x_{m}$ with time at different thermophoretic diffusivities in both intrinsically unstable and stable reference systems along with the inset of the contours of $C_{c}$. The contours are depicted at identical times in each systems. The solid lines represent the systems with $\delta_{T}=0 . \ldots$. . . . . . . . . . 101

5.7 The one dimensional viscosity variation in the reference systems in the presence $\left(\delta_{T}=20\right)$ and the absence of thermophoresis. . . . . . . . . . . . 102

5.8 The one dimensional NP concentration variation at different $\delta_{T}$ with constant $H_{R}=0.1$, and at two $H_{R}$ with constant $\delta_{T}=20$ including the scaled convective and source/sink terms variation. The representative inset of the contours of $C_{n}$ derived from NLS are attached for $\delta_{T}=0$ and $\delta_{T}=20 \ldots \ldots$. . . . 104

5.9 Variation of $\left(C_{c}\right)_{r_{i}}$ in the representative systems at different thermophoretic

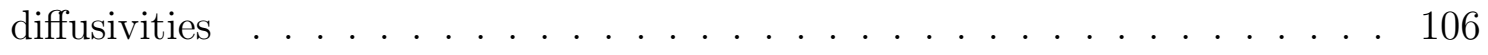




\section{List of Tables}

5.1 Characteristics of classified isothermal systems. . . . . . . . . . . 96 


\section{List of Symbols, Abbreviations and Nomenclature}

Symbol and Abbreviation

2D

$3 \mathrm{D}$

CFT

$\mathrm{CDH}$

EOR

FDM

HDC

HI

ISD

ISU

IUD

IUU

IVC

LSA

ML

NLS

NP

QSSA

RHS

TVF

VF

$A_{s}$

$c_{p}$

$C_{0}$

$C_{a}, C_{b}, C_{c}, C_{j}, C_{n}$

$\bar{C}_{a}, \bar{C}_{b}, \bar{C}_{c}, \bar{C}_{j}, \bar{C}_{n}$

$C_{a}^{\prime}, C_{b}^{\prime}, C_{c}^{\prime}, C_{j}^{\prime}, C_{n}^{\prime}$

$C_{a 0}, C_{b 0}, C_{c 0}, C_{n 0}$

$C_{a, a v}, C_{b, a v}, C_{c, a v}, C_{n, a v}$

$C_{a x}, C_{b x}, C_{c x}, C_{n x}$
Definition

Two Dimensional

Three Dimensional

Colloid Filtration Theory

Cold fluid Displaces Hot fluid

Enhanced Oil Recovery

Finite Difference Method

Hot fluid Displaces Cold fluid

Heterogeneity Index

Intrinsically stable, Downstream

Intrinsically stable, Upstream

Intrinsically Unstable, Downstream

Intrinsically Unstable, Upstream

Initial Value Calculation

Linear Stability Analysis

Mixing Length

Non Linear Simulations

Nano Particle

Quasi Steady State Approximation

Right Hand Side

Thermo Viscous Fingering

Viscous Fingering

Medium aspect ratio

Heat capacity

Initial mass fraction

Mass fraction of the components

Base state mass fraction of the components

Perturbed mass fraction of the components

Initial mass fraction of the components

Transversely averaged mass fraction of

the components

Mass fraction of the components in $\mathrm{x}$ direction 


$$
\begin{aligned}
& \left(C_{c}\right)_{r_{i}} \\
& \left(C_{c}\right)_{t} \\
& d_{p} \\
& D_{a}, D_{b}, D_{c}, D_{i} \\
& D_{b a} \\
& D_{n} \\
& D_{T} \\
& \hat{D_{T}} \\
& \bar{D}_{T} \\
& D a_{d e p} \\
& H_{R} \\
& k \\
& K \\
& K_{B} \\
& k_{d e p} \\
& k_{R} \\
& k_{s}, k_{n f}, k_{m}, k_{p} \\
& L \\
& L e \\
& P \\
& \bar{P} \\
& P^{\prime} \\
& P^{*} \\
& P e \\
& P e_{c} \\
& P e_{T} \\
& r \\
& r_{R} \\
& R \\
& R_{a}, R_{b}, R_{c}, R_{n} \\
& R_{\theta} \\
& t \\
& t^{*} \\
& t_{0} \\
& T \\
& T_{a}, T_{b} \\
& T_{0} \\
& U \\
& V, u, v \\
& \bar{u}, \bar{v} \\
& u^{*}, v^{*} \\
& V_{D} \\
& \\
& \hline
\end{aligned}
$$

Relative production with respect to the case in the absence of thermophoresis Cumulative chemical product

Diameter of the particle Diffusion coefficient of the components Mutual diffusion coefficient Brownian diffusion coefficient Thermometric diffusion coefficient Thermophoretic mobility coefficient

True thermophoretic diffusion coefficient Dimensionless deposition rate

Relative temperature change after the reaction Wave number Permeability

Boltzmann constant Deposition rate coefficient Reaction rate constant Thermal conductivity of the solid, nanofluid, medium and the particle Length of the medium

Lewis number

Pressure

Base state Pressure

Perturbed Pressure

Dimensionless pressure

Péclet number

Solute Péclet number

Thermal Péclet number

Temperature ratio of the displaced fluid to that of the displacing one

Rate of reaction

Viscosity (mobility) ratio

Viscosity (mobility) ratio of the components

Thermal viscosity (mobility) ratio

Time

Dimensionless time

QSSA frozen time

Temperature

Temperature of the components

Initial temperature

Inlet velocity

Velocity

Base state velocity

Dimensionless velocity

Darcy velocity 


$V_{t}$
$W$
$x, y$
$x^{*}, y^{*}$

$x_{m}$
$\alpha$
$\beta$
$\gamma$
$\delta_{a}, \delta_{b}, \delta_{c}, \delta_{j}$
$\delta_{n}$
$\delta_{T}$
$\Delta H$
$\epsilon$
$\theta$
$\bar{\theta}$
$\theta^{\prime}$
$\theta_{x}$
$\lambda$
$\mu$
$\bar{\mu}$
$\mu^{\prime}$
$\mu^{*}$
$\mu_{a 0}, \mu_{b 0}, \mu_{c 0}, \mu_{n 0}$
$\mu_{b f}$
$\mu_{s}$
$\mu_{x}$
$\rho$
$\rho_{b f}$
$\phi$
$\omega$

$\omega$

Thermophoretic velocity

Width of the medium

Coordinates along and transverse to the flow

Dimensionless coordinates along and transverse

to the flow

First moment of the transversely averaged

concentration of the products

Thermal diffusivity

Relative volumetric heat capacity of nanoparticles to that of the medium

Quasi static growth rate

Dimensionless diffusivity of the components

Dimensionless Brownian diffusivity

Dimensionless thermometric diffusivity

Enthalpy

$C_{n 0} \beta$

Dimensionless temperature

Base state dimensionless temperature

Perturbed dimensionless temperature

Temperature in $\mathrm{x}$ direction

Thermal lag coefficient

Viscosity

Base state viscosity

Perturbed viscosity

Dimensionless viscosity

Initial viscosity of the components

Viscosity of the base fluid

Solvent viscosity

Viscosity in $\mathrm{x}$ direction

Density

Density of the base fluid

Porosity

Stream function

Vorticity 


\section{Chapter 1}

\section{Introduction}

Viscous fingering (VF) is a hydrodynamic instability that occurs when a low viscosity fluid displaces a high viscosity one in porous media. At strong enough flow rates, finger-like patterns develop as a result of growing disturbances at the interface of the fluids over time, known as Saffman-Taylor instabilities [1]. The fingering patterns may also grow as a result of gravity mismatch and are known as Rayleigh-Taylor instabilities [2] that develop when a heavy fluid is present on top of a lighter fluid. Such instabilities are encountered in a wide variety of fields. In some applications such as oil recovery process, chromatographic separation, and contaminant transport in soil where a uniform front is desired to achieve the highest volumetric sweep, such instabilities are unfavorable. On the other hand, in microfluidic devices such as micromixers where inertial effects are negligible, VF is an efficient mean to enhance the mixing rate [3]. There are two types of viscous fingering: miscible and immiscible. In the miscible VF the change in the properties which leads to the growth of instabilities results from the injection of a solvent fully miscible with the displaced fluid. On the other hand in immiscible VF, the fluids are immiscible with each other. Consideration of molecular diffusion and mechanical dispersion in miscible displacements distinguishes the system from immiscible displacements where the surface tension must be considered instead.

Different parameters can affect the formation and propagation of these instabilities and 
have been the subject of many studies. In the case of miscible VF which is the focus of the present study, the effects of diffusion [4], permeability heterogeneity [5], heat transfer [6, 7], chemical reaction [8, 9], melting [10], flow conditions [11] and flow configuration [12] have been thoroughly analyzed. The common feature of the above mentioned physical conditions is the alteration of the viscosity distribution, flow conditions or the medium properties to control the instability. Another aspect that has so far received very limited attention is the presence of solid additives in the flow, and more specifically nanofluids that can be injected in porous media. Nanofluids, the suspension of ultra fine nano-sized particles (NP) in a base fluid, are widely used in fluid flow systems [13]. Although nanofluids are well known for their ability for heat transfer enhancement, they are broadly used in other applications as they can affect a number of features of the flow systems. The NP-laden flows that may lead to VF instabilities are observed in applications such as enhanced oil recovery $(\mathrm{EOR})[14,15]$, nano-drug delivery [16] and particle mixing in microfluidic devices [17], to name some. Implementation of NPs as catalysts in chemically reactive flows has also been explored in VF related applications such as heavy oil upgrading [18] and soil remediation [19].

Considering the importance of the problem, the lack of theoretical studies in the field was an incentive to conduct the present study. In this work the effects of the presence of NPs in the formation and the growth of instabilities in miscible systems are investigated. The problem is solved either with linear stability analysis (LSA) or direct non-linear simulations (NLS) of the governing equations. LSA is a strong method to predict the growth of disturbances at early stages of their development. However, NLS lead to a full analysis of the flow configuration and help to a further quantitative analysis. In both methods, small disturbances are initially introduced to the system and the response of the system to the induced perturbations is examined at later times.

The study is carried out in three stages, each introducing some aspects of NPs that may affect the growth of instabilities and flow configuration in a specific flow condition. In the 
first stage, the flow is under isothermal conditions with an emphasis on the effects of viscosity modification after the addition of NPs, the Brownian diffusion and the deposition of NPs on the porous medium. The second stage focuses on non-isothermal flow displacements that as shall be seen later, triggers an additional NP transport phenomenon known as thermophoresis, whose effects and competition with Brownian diffusion are thoroughly analyzed. In the third stage, the catalytic effects of NPs are investigated in reactive flows under both isothermal and non-isothermal conditions. The results of these three investigations are presented in Chapters 3, 4 and 5 respectively. Chapter 2 however, contains a review of previous related studies. Finally, Chapter 6 concludes the summary of the results followed by some recommendation for future works. 


\section{Chapter 2}

\section{Background}

This Chapter consists of four sections. First some of the important studies about the concept of viscous fingering, specifically in miscible displacements, are reviewed. This general overview includes two subsections to further address the effect of two important factors in isothermal flows: diffusion/mechanical dispersion and permeability heterogeneity. Establishing an understanding of the general features of this phenomenon, the review is then extended to address the effects of heat transfer and chemical reaction on miscible VF instabilities in NP-free systems, aligned with the investigation of their effects on NP-laden systems in Chapter 4 and 5 respectively. Finally, in the last section, some studies are presented to discuss some of the already reported effects of NPs on VF instabilities.

\section{$2.1 \quad$ Viscous fingering phenomenon}

The first scientific study of VF can reasonably be attributed to Hill (1952) [20]. Considering a fluid with viscosity and density of $\mu_{2}$ and $\rho_{2}$ on top of another fluid with $\mu_{1}$ and $\rho_{1}$, he defined a critical velocity $u_{c r}=\frac{g k\left(\rho_{1}-\rho_{2}\right)}{\left(\mu_{1}-\mu_{2}\right)}$ to predict the instability. $k$ is called the resistant constant which is determined experimentally. He concluded that if the velocity exceeds $u_{c r}$ the system with $\rho_{1}>\rho_{2}$ and $\mu_{1}>\mu_{2}$ is unstable while it is stable if $\rho_{2}>\rho_{1}$ and $\mu_{2}>\mu_{1}$. After this prominent work by Hill, the attention of many scientists was drawn to this 
phenomenon. There were a couple of studies in 1950s regarding this phenomenon, however the most significant development can be found in the works of Chouke et al. (1958) [21] and Saffman and Taylor (1958) [1], both on immiscible displacements. Chouke et al. carried the first rigorous stability analysis of viscous fingering and found the stability condition and the corresponding critical wave length for the initiation of the instability. They further conducted experiments in a Hele-Shaw cell to validate the results of the stability analysis. The experimental results were in good agreement with the analytic predictions. Finally, they conducted further experiments in a porous medium to observe the differences in finger configurations of the Hele-Shaw cell and the porous medium, and to further analyze the effect of surface tension. Their results show that with the same displacing fluid, when the viscosity of oil (displaced fluid) is higher, smaller fingers are formed. Furthermore, the lower the bulk interfacial tension, the finer the fingers. These results are in accordance with their presented theory. The stability of the immiscible flows were similarly analyzed by Saffman and Taylor in a parallel study [1]. Through the experiments however, they investigated the evolution and the shape of the dominant finger.

In a prominent classical study, Tan and Homsy (1986) [22] conducted a linear stability analysis (LSA) of a miscible binary system. This is a distinguished work, as the previous attempts to solve the problem by considering the time-dependant diffusion base state (one dimensional flow in the absence of perturbations) had serious limitations. By introducing a new correlation for the viscosity as a function of concentration, they solved the problem both analytically and numerically using quasi steady state approximation (QSSA) and initial value (IV) calculations. The QSSA, which was first proposed by these authors, is based on the fact that the rate of change of the base state is much smaller than the growth of perturbations. On the other hand IV calculation approximates the exact solution of the governing partial differential equations. Their results show that the system is unstable for an unfavorable $\log$-viscosity ratios, namely if $R=L n \frac{\mu_{\text {displacing fluid }}}{\mu_{\text {displaced fluid }}}>0$. It was shown that the growth rate of disturbances has its maximum value at $\mathrm{t}=0$ and decays with time as a result of diffusion. In 
addition, the cut-off wave number, the wave number after which the system is stable, shifts to lower values at later times.

The non-linear simulations (NLS) of the phenomenon in miscible displacements was first successfully conducted by Peaceman and Rachford (1962) [23] using the finite difference method (FDM). Their simulated fingers demonstrated good qualitative similarities with experiments. However, due to the limitations in FDM, they were not able to capture the early developments of the fingers. FDM has some limitations such as large numerical dispersion in coarse mesh size, requires more grid points and has difficulty in treating the non-linear terms. Attempts to avoid the mentioned disadvantages in FDM led to taking advantage of other numerical techniques namely the spectral methods. The first NLS with spectral method is attributed to Hatziavramidis (1987) [24] who used Chebyshev expansion to solve the problem. However, his results were not in good agreement with many experimental studies especially at high Péclet numbers.

A year later, a Fourier spectral method was used by Tan and Homsy (1988) [25] to conduct NLS of a miscible displacement of a binary system in a rectilinear Hele-Shaw cell. Using this technique, it was possible to solve the problem up to $P e=500$ with acceptable accuracy compared to the results of LSA at early times. The authors reported that the Péclet number and mobility ratio are the two parameters that determine the stability characteristics. They noted that as time passes, the fingers become wider while their number decreases. This phenomenon called spreading, reflects the fact that the wavelength of fingers shifts with time to a larger spectrum. Furthermore, some fingers shield the growth of the adjacent fingers and collapse with others to form a larger one (shielding phenomenon). They attributed the spreading and shielding phenomena to span-wise secondary instabilities that are aided by the transverse dispersion and the resulting cross flow. Another phenomenon referred as tip-splitting, where the tip of a finger splits to form more fingers, was also observed in their numerical study. The authors attributed the splitting phenomenon to the steep concentration gradient at fingertip as a result of a stretching caused by cross flow. Accordingly, the tip- 
splitting mechanism in miscible displacement differs from immiscible displacement as the flow field at the finger front needs to be stretched and become steep before a finger can split. Finally, they examined the mixing length (ML) in this problem. The ML is the length in which the transversely averaged concentration of one component is in a specific range along the channel, typically $0.01<C_{a v}<0.99$. They reported that the dependence of the ML on time is determined by two mechanisms: at early stages dispersion cause the ML to grow like $t^{\frac{1}{2}}$ and at later times the non-linear fingers cause the ML to grow linearly with time.

Zimmerman and Homsy (1992) [26] studied the 3D miscible VF using the Hartley based spectral method. Their aim was to find any differences both in fingering mechanisms and the average quantities with previous $2 \mathrm{D}$ works. They reported that the $2 \mathrm{D}$ simulation captures the most important physics of the miscible displacement observed from 3D simulations. In both 2D and 3D simulations, the noise in modes outside the cutoff wave number is rapidly damped to the precision limit of the simulation. Furthermore, the shielding, spreading and coalescence (side attachment of one finger to another) observed in 3D simulations occur at $P e$ numbers comparable to those in 2D simulations. They also observed the tip-splitting but at higher $P e$ numbers compared to that observed in the 2D simulation. Furthermore, the 3D longitudinally averaged concentration profiles show the same trend of a decrease in the number of fingers at later times as a result of shielding and spreading mechanism as observed in the 2D simulations. However, at specific $P e$ numbers, the final number of fingers in $3 \mathrm{D}$ simulations was larger than that observed in the $2 \mathrm{D}$ ones. This was because the 3D fingers tend not to travel sideways and as a result, experience less coalescence with respect to the closed 2D fingers. Finally, the asymptotic ML in the 3D simulations derived from the transversely averaged concentration profiles was found to be approximately the same as that in the $2 \mathrm{D}$ simulations.

A recent comprehensive study about miscible displacements in a binary system was conducted by Nijjer et.al (2018) [27]. They reported that the key dimensionless parameters to study the flow are the $P e$ number and the log-viscosity ratio $\mathrm{R}$ defined above. The flow is 
unstable at $R>0$, but only beyond a critical $P e$ number. The value of the critical $P e$ number decreases as $R$ increases. The analysis introduced three distinct regimes in the unstable flow: 1. early time, linearly unstable regime 2. intermediate time non-linear regime and 3. late time, single-finger exchange-flow regime. At early times, series of fine fingers start to develop while growing exponentially with time. In this regime the number of fingers is proportional to both the log-mobility ratio and the Pe number, $n \propto R P e$ while the mixing length grows as $M L \propto\left(\frac{t}{P e}\right)^{\frac{1}{2}}$. After some times the flow enters the non-linear regime where the fingers interact non-linearly through different mechanisms such as fading, shielding, coalescence, tip-splitting, etc. The transition occurs at a time proportional to $t \propto O\left(\frac{\phi^{2} D}{R^{2} U^{2}}\right)$ where $\phi$ and $D$ represent the porosity and the diffusion coefficient, respectively. As a result of these fingering interactions, the number of fingers decreases with time as $n \propto\left(\frac{t}{P e}\right)^{-\frac{1}{2}}$ till it reaches a single finger while the mixing length grows as $M L \propto R t$. Finally, in the third regime $n=1$ and the pair of counter-propagating fingers develop at both ends of the channel but slow exponentially with time. This occurs at a transition time proportional to $t \propto \frac{W^{2}}{D}$ when the instabilities diffuse across the entire width of the porous medium $W$. The mixing length remains constant in this regime with $M L \propto R P e$.

The aim in this review was to address the most important features of binary miscible displacements. This preliminary review leads to the following conclusions: 1. A 2D analysis of VF can capture almost all the physical properties of the problem and so there is no need to use the complicated and time consuming 3D analysis. 2. The LSA with QSSA method is a simple and at the same time very strong tool to predict the instability of the system. 3. The Spectral method has lots of advantages to model the miscible flows. 4. The miscible flow in a homogeneous porous medium is unstable when the viscosity of the displacing fluid is smaller than the displaced one $(R>0)$ if the $P e$ number exceeds a critical value. 5 . Diffusion is a stabilizing factor and the flow rate (injection velocity) is a destabilizing one. 6. In a diffusion dominant early time regime, the ML grows as $t^{1 / 2}$ while in the convection dominant non-linear regime it grows linearly with time. 7. The system finally reaches a 
single-finger growing regime while slowing exponentially and the ML becomes constant.

\subsubsection{Diffusion/Dispersion in miscible viscous fingering}

In earlier studies it was assumed that the transverse diffusion is not an important factor to change the finger configuration. For example, Handy (1959) [28] attributed the claimed neutral effect of diffusion to the fact that the distance between growing fingers is large compared to the diffusional distance.

This conclusion was subsequently revised by Slobod and Thomas (1963) [29]. These authors studied the effects of transverse diffusion on finger growth and the sweep efficiency in a homogeneous porous medium. They used the X-ray technique to observe the shape of the fingers. In order to study the effects of diffusion, they conducted a series of experiments at two different flow rates: high and low. The lower flow rate represented the case with higher residence time in the porous medium where the diffusion had enough time to act, while the opposite is true for the higher flow rate. They observed that at fast flow rates, initially generated fingers merge and finally reach to a couple of fingers in the middle of the domain. On the other hand at the slow flow rate, the fingers appear initially so late and finally merge to a single bulging finger. Accordingly, they concluded that in contrast to the earlier studies, the transverse diffusion can move materials across the flow directions between fingers if they have enough residence time. This further led to a conclusion implying that transverse diffusion has a stabilizing effect.

The LSA of Tan and Homsy (1986) [22] validated the stabilizing effect of diffusion such that the system was less unstable at larger base-state freezing time in QSSA where the system had more time to diffuse. Furthermore, the analysis showed that the diffusion attenuates the disturbances with wave numbers greater than a finite value (cutoff). As known, the dispersion

tensor is generally anisotropic and velocity dependent. Accordingly, extending the study to consider anisotropic dispersion illustrated that the small transverse dispersion increases the growth rate with respect to the isotropic case while the large transverse dispersion tends to 
stabilize the system.

Yortsos and Zeybek (1988) [27] conducted LSA of a miscible binary system focusing on the dependence of the anisotropic dispersion on velocity. They reported that the strong velocity-dependent longitudinal dispersion causes the system to become more unstable at short wavelength. This effect is highly pronounced close to the onset of the displacement.

Zimmerman and Homsy (1991)[30] adopted a Hartley-based spectral method to conduct NLS of this problem. Their dispersion coefficient was coupled with the velocity field. They observed multiple coalescence and fading mechanisms in the non-linear finger growth with lateral dispersion which had not be previously reported in the case of isotropic dispersion. Using vortex analysis, they explained in particular the pairing mechanism. According to the pairing mechanism, the number of vortices and hence the number of fingers in the linear regime decreases by almost one-half before any macroscopic fingers appear. This is because the uneven cross-flow generated by the transverse dispersion merges the neighboring vortices of opposing spin or adjacent fingers. This allows one finger to spread and as a result shield the growth of the neighboring finger. Accordingly, the slow growing finger disappears in the wake of the fast growing one and the number of fingers decreases while their strength increases. The authors further investigated the rate of growth of the ML in this system. They reported that the ML at early times and late times grows as $t^{\frac{1}{2}}$ and $t$ respectively, similar to what were already known in the isotropic diffusive case. Their further analysis shows that at constant $R$ and high enough values of $P e$ numbers, the asymptotic mixing rate is independent of the dispersion anisotropy and the Péclet number. In another study [31], the same authors reported that the asymptotic mixing rate increases monotonically with the viscosity ratio $R$.

In another prominent work, Manickam and Homsy (1993) [32] found out that the flows that are predicted to be stable with step profile approximation in a system with nonmonotonic viscosity distribution, become unstable at later times as the base state diffuses out. This was in contrast to what had been expected, in that diffusion has always a sta- 
bilizing effect. This study reveals that systems with non-monotonic viscosity profiles can become unstable regardless of the value of the end-point viscosity as a result of diffusion. They further determined the critical time at which the stable system becomes unstable, as a function of the parameters of the model viscosity profile.

Ghesmat and Azaiez (2008) [33] investigated the effect of velocity dependent anisotropic dispersion. The model they used contained a velocity dependent transverse dispersion which had been ignored and simply substituted by diffusion in previous studies. Their results show that the finger structure in flows with isotropic velocity dependent dispersion is similar to those observed in a diffusive flow. However, the anisotropy in the dispersion has considerable effects on the finger configuration and the interaction mechanisms between adjacent fingers. Supported by a quantitative analysis, they concluded that as the ratio of the transverse to longitudinal dispersion decreases, the system becomes more unstable with more intricate finger configuration. Accordingly, the complexity of the system is mitigated as the effect of the transverse dispersion increases.

If the diffusivities of the acting miscible solutions are different, double diffusive effects may lead to interesting results. A comprehensive study of such systems was conducted by Mishra et al (2010) [4]. Considering a fluid A with viscosity ratio and diffusivity of $R_{a}$ and $D_{a}$, displacing a solution B with corresponding properties of $R_{b}$ and $D_{b}$, the system is intrinsically unstable if $R_{b}-R_{a}>0$ regardless of the values of diffusivities. However, according to their study, the opposite trend with $R_{b}-R_{a}<0$ does not always imply a stable flow. Their LSA at $t_{0}=0$ showed that in this scenario, the system becomes unstable if $\delta<\frac{R_{b}}{R_{a}}$, while $\delta=\frac{D_{b}}{D_{a}}$. Further analysis at $t_{0}>0$ showed that this system can actually become unstable asymptotically if $\delta<\left(\frac{R_{b}}{R_{a}}\right)^{\frac{2}{3}}$. It should be noted that $R_{b}$ and $R_{a}$ were both taken to be positive in their study. This conclusion was further validated through a series of NLS.

This brief review leads to the following conclusions: 1 . The mutual diffusivity $D$ in a binary system has actually a stabilizing effect. 2. Systems with non-monotonic viscosity 
distributions become unstable with the contribution of diffusion. 3. The flow configuration of the system with isotropic velocity dependant dispersion is similar to that observed in the diffusive flows. 4. As the ratio of the longitudinal to transverse dispersion increases, the system becomes more unstable compared to the diffusive system and vice versa. 5 . The viscously stable system (more viscous fluid displaces a less viscous one) may become unstable as a result of the double diffusion effects of the approaching solutions.

\subsubsection{Permeability heterogeneity in miscible viscous fingering}

Most of the studies on VF instability have focused on homogeneous porous media. Experimental works are mostly conducted in Hele-Shaw cells as a representative of homogeneous porous media, since it is easy to set up and also to visualize the finger growth and their interactions. However, in practice the porous medium may be heterogeneous and therefore the variation of the microstructure of the medium may change the permeability. The change in the permeability distribution of the porous medium leads to two instability mechanisms: 1. mobility induced bypassing termed 'fingering' and 2. permeability related bypassing termed 'channeling' [34].

The first numerical study of the displacement in heterogeneous porous media is attributed to Peaceman and Rachford (1962) [23], although the focus of their study was not the heterogeneity of the medium. The simulated finger configuration was qualitatively in a good agreement with the experimental results of Blackwell et al. (1959) [35]. However the results could not capture the early growth of the fingers.

Araktingi (1988) [36] used particle tracking to simulate miscible displacements in heterogeneous porous media. He took advantage of the heterogeneity index $\left(H I=\left(\sigma_{L n K}\right)^{2} \lambda_{D}\right)$ to measure the heterogeneity of the medium. $\left(\sigma_{L n K}\right)^{2}$ was the variance of the log normal permeability distribution and $\lambda_{D}$ represented the degree of correlation between the permeability at two points of the medium. Based on the value of $H I$, he introduced two types of flow patterns. For a small value of $H I$, the finger patterns were found to be similar to those 
observed in homogeneous systems. On the other hand, if $H I$ was large, the growth of the fingers was dominated by the permeability distribution. Accordingly, in this case the fingers grew through the high permeability regions regardless of the value of the mobility ratio. The author further investigated the validity of Koval's assumption for these flow regimes. According to Koval's assumption, the rate of growth of ML is constant after a period of time. It was found that this assumption is valid for mobility dominated heterogeneous systems (small $H I$ ), while it is not valid for permeability dominated systems (large $H I$ ).

The interaction between permeability heterogeneity and the viscosity-mismatch driven instabilities was well depicted in the pioneering theoretical work of Tan and Homsy (1992) [37]. Their permeability field was modeled by statistically stationary random function with Gaussian distribution. They concluded that in addition to $R, P e$ and $A_{s}$ (the domain aspect ratio), the dynamics of the system were governed by an additional three dimensionless groups, namely $s$ (the variance of the permeability) and $(l x, l y)$ the longitudinal and transverse correlation scales. They found that at relatively small correlation scales $(l x=0.2, l y=$ 0.2 ), although the special variation of the permeability affects the specific path taken by the fingers, the non-linear dynamics of the system still continue to be governed by the mobility ratio. Furthermore, with this length scale, the ML varies linearly with time in the heterogeneous porous media similar to what is observed in homogeneous media. However, the convective mixing rate is not constant anymore and increases with the variance of the permeability $s$.

Tchelepi et al. (1993) [38] studied the effect of permeability heterogeneity on miscible VF. The concentration histories obtained by acoustic measurements were compared with the numerical simulations conducted using the particle tracking method. Millstone and limestone cores were used as the porous media, where limestone was much more heterogeneous than the millstone. The results show that when the mobility ratio $(M)$ decreases below unity, the concentration distribution follows the dispersive behavior in both porous media and so, the displacement suppresses the effects of heterogeneity. They attributed this behavior to 
the small longitudinal dispersivity, $\alpha_{l}$. Their calculations show that in the relatively more heterogeneous porous medium, $\alpha_{l}$ is very small when $M<1$ compared to the case with $M>1$. In the unstable situation $(M>1)$ their $2 \mathrm{D}$ simulations show very good agreement with both experimental observations and 3D simulations in homogeneous and relatively less heterogeneous systems. In the case of the relatively more heterogeneous porous medium however, there is a considerable disparity between the $2 \mathrm{D}$ simulations and the experimental observations. However, the authors claimed that the results are still acceptable and therefore the $2 \mathrm{D}$ simulations of a relatively more heterogeneous porous medium can lead to fairly acceptable results.

In the previous studies, the permeability was modeled with a random function. Although the random distribution of the permeability is a good representation of a real case and gives very useful information, it has limitations when it comes to interpreting the fundamental mechanisms of interactions between the viscosity driven fingering and the heterogeneous permeability distribution. Accordingly, in order to resolve this limitation, De Wit and Homsy (1997) [39, 40] introduced a simple spatially periodic model to define the heterogeneous permeability. In their NLS they chose an initially viscosity-driven unstable system and looked for the effects of permeability on fingering patterns and their growth rate. Their tool was to change the magnitude of the amplitude $(\sigma)$ and the wave numbers of the medium heterogeneity $\left(n_{x}, n_{y}\right)$. They chose two systems to study, namely the layered system $\left(n_{x}=0\right.$ and $n_{y}$ varies) and the checkboards (both $n_{x}$ and $n_{y}$ vary). It was found that in the layered system there is a critical value of $\sigma_{c r}$ such that the fingering regime is changing. $\sigma_{c r}$ depends on the values of $P e$ and $n_{y}$. In the system with $\sigma<\sigma_{c r}$ the regime is convective in which the fingering patterns are analogous to those in homogeneous porous systems with observed shielding, tip splitting, fading, merging, etc. and the ML grows as $t$. On the other hand in systems with $\sigma>\sigma_{c r}$ the regime is dispersive in which the "channeling" occurs and the ML grows as $t^{\frac{1}{2}}$. They further found that channeling occurs through a Taylor dispersion mechanism. By introducing the axial spatial variation of permeability (checkboards system) 
channels were destroyed, tip splitting appeared and the system returned to the convective regime.

Norouzi and Shoghi (2014) [41] investigated the effect of both anisotropic dispersion and permeability in miscible binary systems. From LSA and NLS they concluded that as the ratio of the longitudinal to transverse permeability $\left(\alpha_{k}\right)$ and dispersion $\left(\alpha_{D}\right)$ increases, a less and more unstable flow is obtained, respectively. They justified their findings as follows: as $\alpha_{k} \geq 1$, the initial perturbations cannot easily grow as the fingers usually require transport in the transverse direction. As a result the conditions for finger growth are not well-provided and the flow is less unstable. In addition, as $\alpha_{D} \geq 1$ the effect of transverse dispersion which can moderate the concentration gradient is less pronounced and the flow is more unstable.

In the above mentioned discussion it is concluded that: 1. At very small mobility ratios where the diffusion is dominant, the system still follows the dispersive regime regardless of the degree of the permeability heterogeneity. 2. Depending on the permeability distribution in the porous medium there are two instability mechanisms: fingering and channeling. In the fingering regime, the non-linear dynamics of the flow are still governed by the mobility ratio although the permeability heterogeneity affects the specific path taken by the fingers. However, in the channeling regime, the effect of the mobility ratio is reduced and the flow bypasses through the more permeable paths even under favorable mobility ratios. 3 . The variance of the permeability distribution and its covariance which is characterized by the correlation scales, are the tools to distinguish the instability mechanisms. At small variance and correlation scales the flow is dominated by the mobility ratio. The opposite trend in the variance and the correlation scales of the permeability leads to the channeling regime. 4. In the fingering regime, the ML still grows linearly with time. However, its growth rate increases with the variance of the permeability distribution. 


\subsection{Thermo-viscous fingering phenomenon}

Thermo-viscous fingering (TVF) instability is the term used to refer to displacements where temperature mismatch of the approaching fluids may contribute to trigger, suppress or modify the instabilities. This modification is induced through the viscosity adjustment, different thermal diffusivities of the fluids as well as the difference in the advective velocities of the fluids and thermal fronts. These effects are usually represented by the dimensionless parameters of $\beta_{T}=\operatorname{Ln}\left(\frac{\mu_{T_{2}}}{\mu_{T_{1}}}\right)$, the $\log$ viscosity ratio of the fluids at their initial temperatures, the Lewis number $\left(L e=\frac{\alpha}{D}=\frac{P e_{c}}{P e_{T}}\right)$ which is the ratio of the thermal to solute diffusivity and the thermal lag coefficient $\left(\lambda=\frac{\phi \rho_{f} C_{f}}{\phi \rho_{f} C_{f}+(1-\phi) \rho_{s} C_{s}}\right)$ which represents the relative advective velocity of the thermal front to solute front and is dependent on the properties of the fluids and the porous medium. $\lambda<1$ in a general porous medium while it is equal to one in the Hele-Shaw cell. The general features of this phenomenon is explained below through a review of some of the most representative studies.

The LSA of miscible TVF was conducted for the first time by Pritchard (2004) [42] for radial flows. He conducted the analysis for a) single-front flows where one front is unstable and the other is neutral and b) double-front flows where both or either fronts can be stable or unstable. In the single front analysis, it is reported that with a new scaling of $\alpha=\lambda D$ and $t_{T}=\lambda^{-1} t_{c}$, the re-scaled energy equation becomes identical to the concentration equation. Accordingly, with this scaling the highest eigenvalue or equivalently the most dangerous mode of the perturbations is identical in either of the systems with only the solutal or the thermal front. The double-front stability analysis for unstable solutal fronts and unstable/stable thermal fronts shows that the instability is controlled mainly by the properties of the solutal front due to the much higher solute $P e_{c}$ as opposed to the thermal $P e_{T}$. This implies that the thermal front can only modify the instability of the system which is determined principally by the solutal front. However, for very large values of $\lambda$ or if $\beta_{T}>>\beta_{c}$ ( $\beta_{c}$ is the solute viscosity ratio already shown by $R$ in isothermal flows), the thermal front may have a noticeable effect on the instability. The final case in which the solutal front is weakly stable 
but the thermal front is unstable, indicates that the fluid front cannot stabilize the instability centered on the thermal front, although it provides a barrier to its growth. In summary, the study concludes that the stability of these systems must be considered separately on each front. If either of the fronts is unstable, then the instability is likely to grow although the rate of its growth is modified significantly due to the double-front coupling effects.

Both LSA and NLS of TVF instabilities in the rectilinear geometry were carried out in separate studies by Islam and Azaiez (2010) [6, 7]. The stability analysis was conducted through both QSSA and IVC methods. It is worth stressing that in the IVC method, the linearized governing equations were solved directly as apposed to QSSA where the equations were solved at a fixed time. It is reported that at $L e=1$, and $\lambda=1$ the flow is equivalent to the isothermal one with the effective viscosity ratio of $\beta_{\text {eff }}=\beta_{c}+\beta_{T}$. This implies that as $\beta_{T}$ increases the flow is more unstable. Investigating the behaviour of the system for $\beta_{c} \geq 0$ and $\beta_{T} \geq 0$ showed that $\beta_{T}$ still has a noticeable destabilizing effect when $\lambda<1$ but at $L e=1$. This effect was observed even for very small values of $\lambda$. However, both the maximum growth rate and the cut-off wave number were reduced as $\lambda$ decreases, implying that the system becomes less unstable with decreasing $\lambda$. Extending the study to investigate the effect of $\beta_{T}$ at $\lambda=1$ but in large Le numbers showed differences in the predictions of the two methods of stability analysis. According to QSSA, $\beta_{T}$ still had a destabilizing effect at large Le, while the opposite trend was found with IVC. However, comparing the results with the NLS, the authors confirmed the validity of the results of IVC. It should be noted that in both methods the maximum growth rate was found to decrease at higher Le compared to the case where $L e=1$, implying less unstable flows with increasing Lewis number. These results were derived when both $\beta_{c}$ and $\beta_{T}$ are positive. The contours of concentration when $\beta_{c}>0$ but $\beta_{T}<0$ show that the system is now more unstable at higher Le compared to the case when $L e=1$. It implies that the effect of $L e$ is dependent on whether a hot fluid displaces a cold one or vice versa. Finally, the authors reported that the coupled effect of very large $L e$ and small $\lambda$ almost eliminate the destabilizing effect of $\beta_{T}$. 
In the previous studies it was reported that at $L e=1$ the non-isothermal system becomes unstable if $\beta_{c}+\beta_{T}>0$ while it is stable otherwise. However, different diffusivities of the mass and heat $(L e>1)$ may lead to interesting counter-intuitive results. This effect, known as "double diffusion" was examined thoroughly by Mishra et al. (2010) in the case of a Hele-Shaw cell $(\lambda=1)$. Some of the important conclusions derived from LSA and validated through NLS, are summarized in Fig. 2.1. In this figure $R_{s}$ and $R_{f}$ represent the $\log$ viscosity ratios of the slow (fluid) and fast (heat) diffusing components respectively, while $\delta=L e=\frac{D_{f}}{D_{s}}>1$. Depending on the values of $R_{s}, R_{f}$ and $\delta$, the study introduces six different profiles for the viscosity distribution and accordingly for finger configurations. It is reported that the system is always unstable if $R_{f}+R_{s}>0$, however the opposite case of $R_{f}+R_{s}<0$ represents either stable or unstable situation. The analysis shows that regions $I V_{d}$ and $V$ become immediately unstable $(t=0)$ while the other regions where $R_{f}+R_{s}<0$ are stable. However, as time passes, the unstable region expands in $\left(R_{s}, R_{f}\right)$ domain to include regions $I I I$ and $I V_{c}$. Then asymptotically only regions $I V_{a}$ and $I V_{b}$ defined by $R_{f}<-\delta^{\frac{3}{2}} R_{s}<0$ are stable.

Including the effect of thermal lag coefficient $(\lambda<1)$, Azaiez and Sajjadi (2012) [43] investigated double-diffusive double convective effects in miscible displacements. Fig. 2.2 summarize some of the results of their study. In the figure, $\beta_{1}$ and $\beta_{2}$ represent the solutal and thermal log viscosity ratios respectively. It is demonstrated that the flow is instantly unstable as long as $\beta_{1}+\lambda \beta_{2}>0$. In case where $\beta_{1}+\lambda \beta_{2}<0$, the flow will still be unstable at $t=0$ under the condition that $\delta \beta_{1}+\lambda \beta_{2}>0$. This result implies that in the case of a pure thermal displacement, the system is unstable as long as $\beta_{2}>0$ and $\lambda>0$, however as $\lambda$ decreases the system gets less unstable. Interestingly, with $\beta_{1}=\beta_{2}$ the system with single solutal front is more unstable than that with a single thermal front, as long as $\lambda<1$. It is also reported that at $t>0$ the unstable region expands in the $\left(\beta_{1}, \beta_{2}\right)$ domain in which, asymptotically only the shaded region with $\beta_{1}<0, \beta_{2}<0$ is stable. The other important result is that as $\lambda<1$ decreases, the instantaneous unstable region in the quadrant $\left(\beta_{1}>0\right.$, 


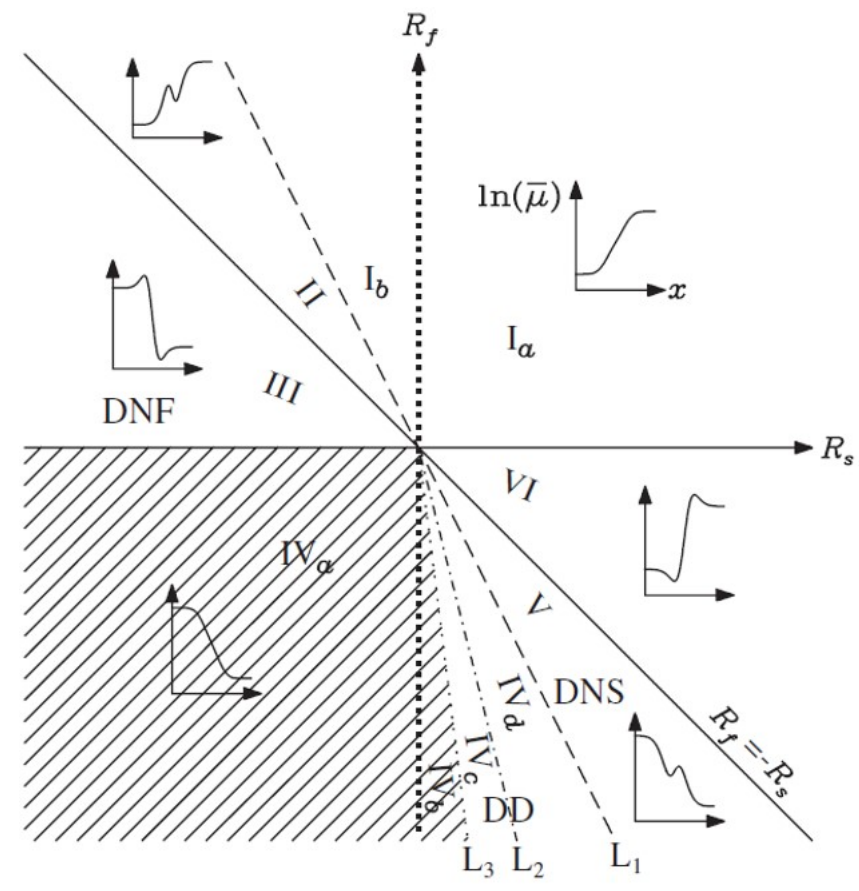

Figure 2.1: $\left(R_{s}, R_{f}\right)$ plain of six different base state viscosity distributions in double diffusive miscible displacements reported by Mishra et al. [4]. $R_{s}$ and $R_{f}$ are the log viscosity ratios of the slow and fast diffusing components while $\delta=\frac{D_{f}}{D_{s}} . L_{n}(n=1,2,3)$ denotes lines of $R_{f}=-\delta^{\frac{n}{2}} R_{s}$

$\left.\beta_{2}<0\right)$ expands while it shrinks in quadrant $\left(\beta_{1}<0, \beta_{2}>0\right)$. Through QSSA, it was found that at higher values of $\delta$ with $\beta_{1}>0, \beta_{2}>0$ the characteristic curves for all values of $\lambda$ collapse almost to a single curve, that for the isothermal flow with identical $\beta_{1}$. However interestingly, if $\beta_{2}<0$ and $\beta_{1}>0$ the system is more unstable than the isothermal flow with identical $\beta_{1}$. This implies that the presence of a favorable second component tends to enhance the instability compared to the initially unstable single-component system. This enhancement is more noticeable as $\lambda$ decreases. These results were validated with NLS.

\subsection{Viscous fingering of reactive fronts}

Approaching two reactive fluids may trigger or modify the VF instabilities in porous media. This phenomenon is observed in oil recovery [44], chromatographic separation [45], contam- 


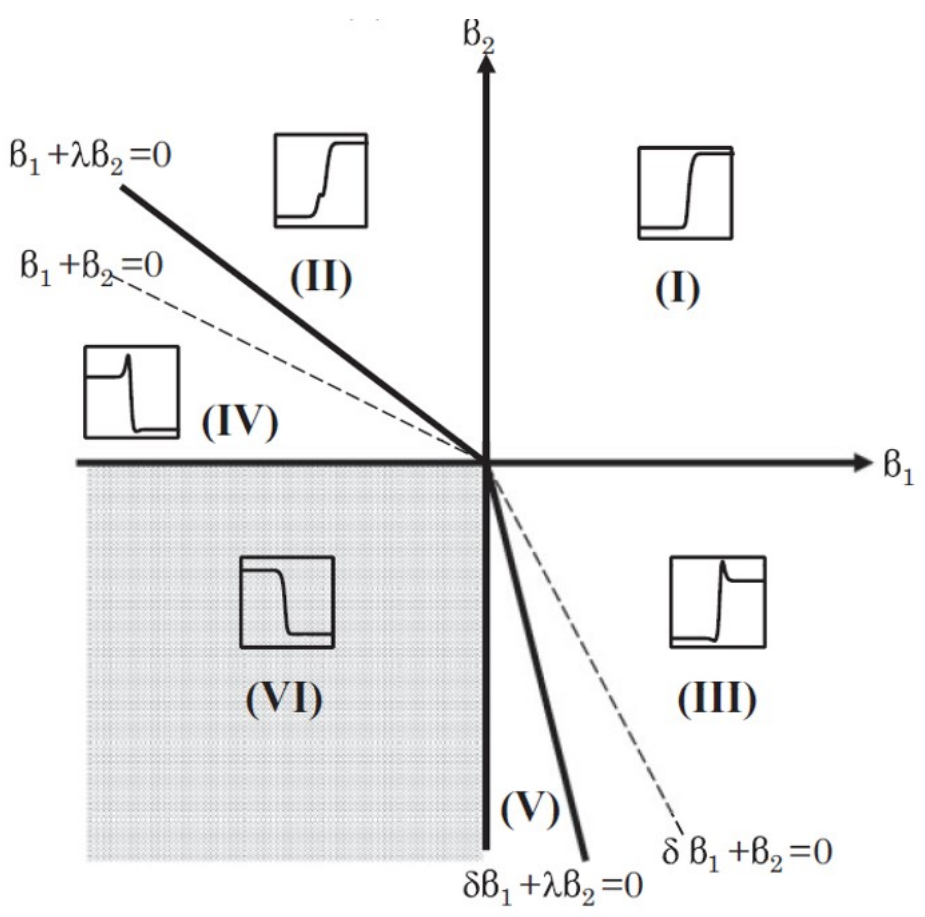

Figure 2.2: The base state viscosity distributions in double diffusive-double convective miscible displacements reported by Azaiez and Sajjadi [43]. $\beta_{1}$ and $\beta_{2}$ are the solutal and thermal $\log$ viscosity ratio respectively, $\delta$ is the symbol used to represent Le number and $\lambda$ is the thermal lag coefficient.

inant degradation [46] and polymerization fronts [47] to name some. Relevant studies were conducted mostly on systems in which the approaching fluids $A$ and $B$ are undergoing either autocatalytic $A \rightarrow B$ or bimolecular $A+B \rightarrow C$ reactions. The dynamics of the system are then modified though the rate of the reaction, the diffusivities of the different components, the velocity of the frontal chemical wave in the autocatalytic reaction and the viscosity of the chemical product $C$ in the bimolecular reaction. The rate of the reaction is reflected through the Damköhler number (Da), representing the ratio of the dispersive time scale to the reaction time scale. Additional dimensionless groups includes $R_{c}$ that represents the $\log$ viscosity ratio of the product $C$ to that of the displacing fluid, and $\delta_{i}$ the relative diffusivities with respect to the displacing component. Below, the most important features of the chemically driven VF instabilities are summarized by referring to some important studies.

The most important features of the systems undergoing an autocatalytic reaction were reported by Dewit and Homsy (1999) in two separate studies [8, 9]. It is observed that 
the initially unstable non-reactive binary system becomes more unstable if the approaching fluids are reactive following autocatalytic reaction in the absence of chemical traveling wave. Specifically, increasing both the mobility ratio of the fluids and $D a$ intensifies the instability. An increase in the mixing zone of the fluids, enhancement of the tip splitting and formation of droplets are some of the other features observed in the presence of chemical reaction. However, the droplet formation and the finger propagation may be modified in the presence of a chemical traveling wave.

Non-isothermal autocatalytic reactive systems were examined by Swernath and Pushpavanam (2007) [48]. Heat transfer effects through the temperature dependency of the viscosity in such systems, further increases the instability if the reaction is exothermic and mitigates it if it is endothermic. The Le number on the other hand has an opposite effect such that it increases the instability in endothermic reactions while it suppresses it in exothermic ones.

Most existing studies on chemically driven VF have focused on a simple $A+B \rightarrow C$ reaction where the product $C$ may have a different viscosity than those of the displacing and displaced fluids; A and B. In two separate experimental studies, Nagatsu et al. (2007)(2010) $[49,50]$ investigated the dynamics of the flow in both reactive and non-reactive systems at similar unfavorable viscosity ratios. It was shown that the fingering patterns and the area occupied by the fingers are different in those systems, implying the effect of the reaction on the dynamics and the instability with unfavorable viscosity ratios.

Subsequently, Podgorski et al. (2007) [51] observed through experiments that initially stable binary systems can even become unstable as a direct effect of the $A+B \rightarrow C$ reaction. However, although both the invading and displaced fluids had the same viscosity, the fingering patterns were interestingly different depending on which fluid is invading the other. Conducting a theoretical study of such initially iso-viscous binary systems, Gérard and Dewit (2009) [52] reported that the asymmetry in the finger patterns after the reaction emerges as a result of unequal concentrated reactants and/or the difference in their diffusivities.

A thorough LSA of systems undergoing a bimolecular $A+B \rightarrow C$ reaction was conducted 
by Hejazi et al. (2010) [53]. Since the rate of chemical production is time dependent, it is shown that the stability criteria change with time. However asymptotically, it was found that an initially unstable binary system remains unstable after the reaction regardless of the viscosity of the product. In addition, depending on the viscosity of the chemical products, initially stable binary systems can become unstable as a result of the reaction. Specifically, defining $R_{b}$ and $R_{c}$ as the log viscosity ratios of the displaced fluid and the product to that of the invading fluid, it is reported that two zones, namely the trailing and the leading zones, develop in the flow with the effective viscosity ratios of $R_{T}=\frac{R_{c}}{2}$ and $R_{L}=R_{b}-\frac{R_{c}}{2}$ respectively. If either $R_{L}$ or $R_{T}$ is positive, the system would be unstable asymptotically. In other words, the viscosity distribution is monotonically decreasing if $0>\frac{R_{c}}{2}>R_{b}$ and the system is stable. On the other hand it is unstable if $0<\frac{R_{c}}{2}<R_{b}$ where the viscosity is monotonically increasing or if $\frac{R_{c}}{2}\left(R_{b}-\frac{R_{c}}{2}\right)<0$ where the viscosity distribution is nonmonotonic. The large time asymptotic viscosity profile in $\left(R_{b}, R_{c}\right)$ plain reported by the authors is illustrated in Fig. 2.3 in which the stable flow condition is shown in shaded regions.

NLS of such generic systems were carried out for both moderate and infinite $D a$ numbers respectively by Hejazi and Azaiez (2010) [54] and Nagatsu and De Wit(2011) [55]. It is reported that in the diffusive regime, the cumulative amount of products increases with time as $t^{\frac{1}{2}}$. However, the rate of the production in the convective regime was shown to be increased, indicating that the development of the fingers increases the mixing and in turn the production rate. Furthermore, fingering patterns were analyzed according the already classified stability conditions introduced in the LSA. It is observed that in the intrinsically unstable system $\left(R_{b}>0\right)$, the fingering patterns after the reaction develop at both the leading and trailing fronts, however they advance more in the trailing zone if $R_{T}>0$, $R_{L}<0$ and vice versa. On the other hand, in those intrinsically stable systems $\left(R_{b}<0\right)$ that the instability develops after the reaction, the fingering patters are less noticeable at either of the zones with favorable viscosity ratios. Therefore, the fingers are more likely to 


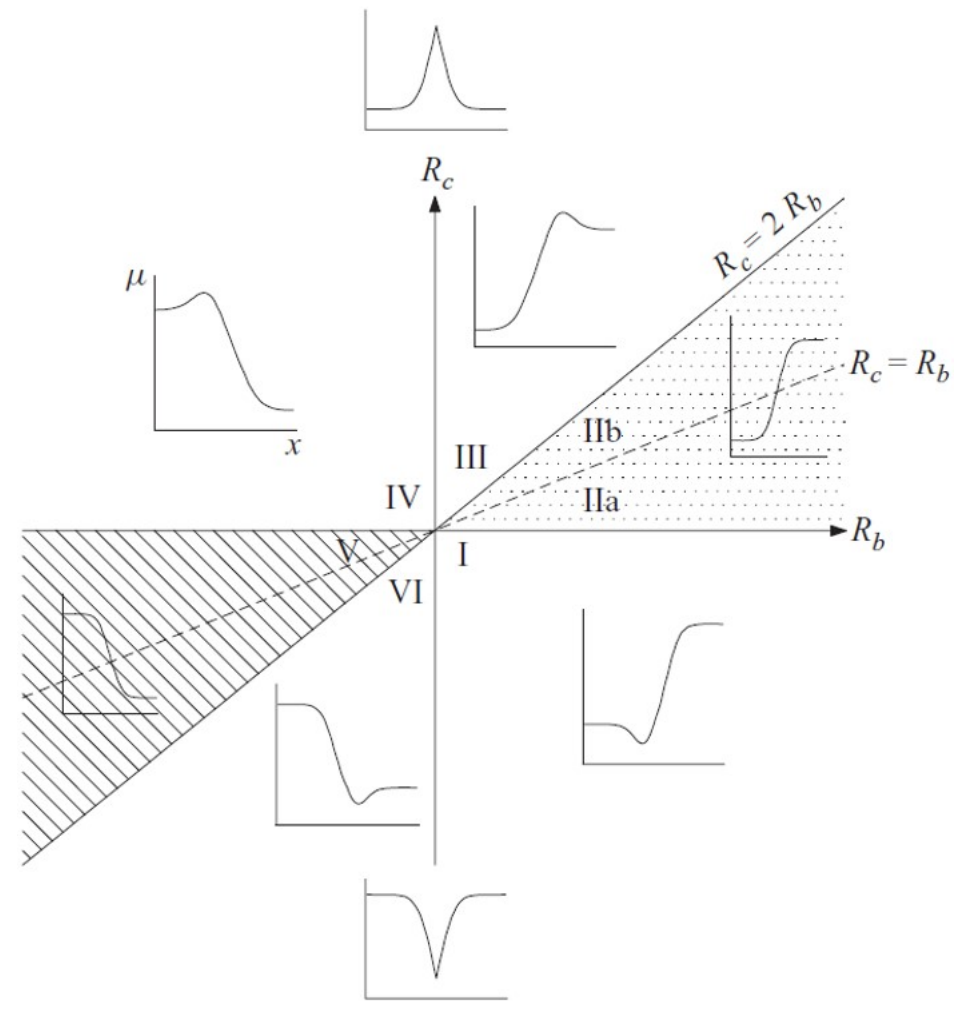

Figure 2.3: Large time viscosity profiles of reactive systems with identical component diffusivities undergoing $A+B \rightarrow C$ reaction in $\left(R_{b}, R_{c}\right)$ plane. [53]

develop at the opposite zone in which the viscosity ratio is unfavorable.

\subsection{Controlling viscous fingering with nanoparticles}

As a result of the fast growing applications of nanotechnology, nanofluids, which can affect the initial features of the flow systems, are widely used in different applications. In enhanced oil recovery for example, the utilization of nanofluids is growing because they are very efficient. In comparison to conventional colloids, nanoparticles (NPs) can flow through the porous medium and remain stable in the reservoir under harsh conditions of high pressure, temperature, and salinity. It is also well-known that they can increase the viscosity, thermal conductivity and the convective heat transfer coefficient of the base fluid [56-58]. Their ability to control the mobility ratios of the fluids [59], to reduce the interfacial tension [14], 
to change the wettability of the porous medium [60,61], to modify the self-diffusion [60] and the relative permeability of the components [62] has been reported and discussed in several studies. This raises the question of the potential effects of the presence of NPs on the instability, specifically the miscible VF instabilities that is the focus of the present study. However, despite the importance of this question, there are only very few studies on the topic, dealing mainly with miscible displacements. Below the related studies are presented. In these studies the nanofluid is injected to displace another fluid, fully miscible with the injected one, in a porous medium or a Hele-Shaw cell.

Ghesmat et al. (2011) [63] conducted an LSA of an initially unstable isothermal system after the addition of NP to the displacing fluid. It was reported that the addition of NPs attenuates the instability. Specifically as $R_{n}$, the viscosity ratio of the nanofluid to that of the base displacing fluid, increases the system becomes less unstable. NP deposition was however reported to enhance the instability. Regarding the effect of NP diffusivity, the study shows a turning point in the instability. Specifically, Brownian diffusivity was shown to attenuate the instability if it is smaller than the diffusivity of the fluids, while make the system more unstable otherwise. In another study [64] in (2013), the authors conducted an LSA of this system, but with reactive fluids undergoing an autocatalytic reaction $A+n \rightarrow B+n$ where the NPs act as nano-catalysts. It is shown that with the set of parameters used, both the Damköhler number $D a$ and the concentration of NPs enhance the instability. Furthermore, similar to the non-reactive systems, it is reported that the NP deposition enhances the instability while Brownian diffusivity has two opposing effects on the instability.

In a recent study by Zargartalebi and Azaiez (2018) [65] on the effects of NPs at the mesoscopic scale for non-isothermal porous media consisting of regularly arranged impermeable disks, it was found that both the NP size and surface potential contribute to an enhancement of the instability. It was also reported that for any given surface potential, NPs are no longer able to affect the instability when their size exceeds a critical diameter.

Both LSA and NLS of the autocatalytic reactive isothermal systems have been investi- 
gated by Sabet et al. (2018) [66] in the presence of dispersed nano-catalysts in the invading fluid. It is reported that the increase in the viscosity of the invading fluid after the addition of nano-catalysts mitigates while the reaction rate promotes the instability. It is shown that NP deposition at $R_{n}>0$ may lead to two opposing behaviors in terms of stability and mixing rate. At small enough $R_{n}$, deposition is shown to be led to less unstable/mixed situation. However, at large enough $R_{n}$, deposition makes the system more unstable/mixed. 


\section{Chapter 3}

\section{Dynamics of Isothermic Nano-flow Displacements in Porous Media}

${ }^{1}$ In the present study, the effects of different parameters on VF instabilities in the presence of non-reactive nanoparticles (NPs) are analyzed. First the LSA (linear stability analysis)based problem will be solved analytically at $t=0$ to predict which parameters can have a role in the growth of instabilities. Furthermore, the long wave instability and the cutoff wave number analyses will be conducted to predict some features of the flow system. Subsequently, the problem will be carried out numerically at larger times with both LSA and nonlinear simulations (NLS), and the results will be explained broadly.

\subsection{Mathematical model}

\subsubsection{Physical problem}

A two dimensional miscible displacement of two fluids in a homogeneous porous medium with constant porosity and permeability is considered in which the displacing fluid contains

\footnotetext{
${ }^{1}$ This chapter is based on the following journal article:

B. Dastvareh, J. Azaiez, "Instabilities of Nanofluid Flow Displacements in Porous Media", Physics of Fluids, vol. 29, (2017) 044101.
} 


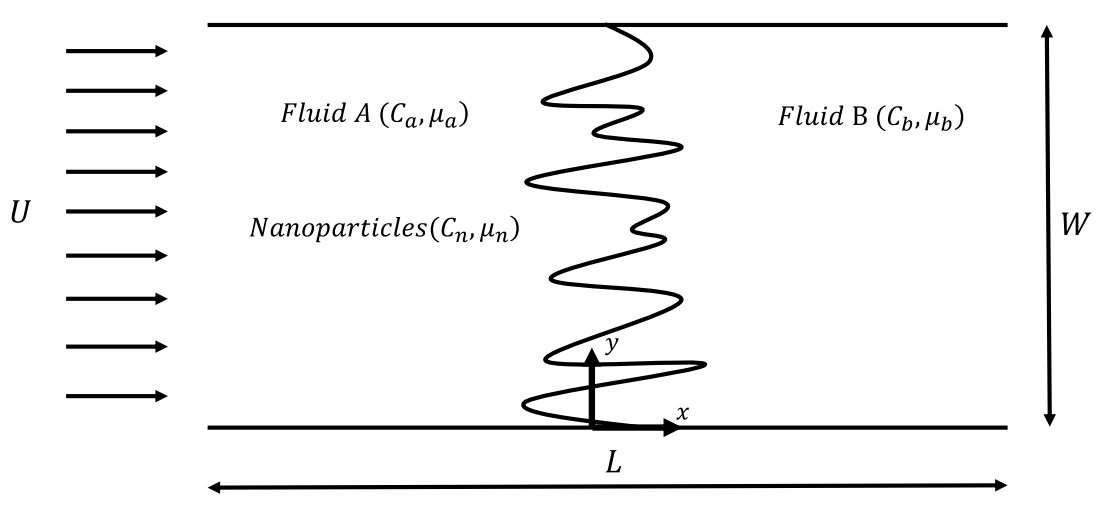

Figure 3.1: Schematic view of the medium

NPs. Fluid A with initial concentration $C_{a 0}$ and viscosity $\mu_{a 0}$ is injected with the constant velocity $U$ along the $x$ axis to displace fluid B with the equivalent properties; $C_{b 0}$ and $\mu_{b 0}$. Fluid A contains NPs with the concentration $C_{n 0}$ and viscosity $\mu_{n 0}$ which is the viscosity of the base fluid after the addition of NPs. For the three components in the system, the concentrations of each component are defined with reference to a base fluid. The base fluid is the solvent to which components A, B and the NPs are added. Furthermore, as a result of this multicomponent system, the diffusion coefficients of each component in the base fluid are $D_{a}, D_{b}$ and $D_{n}$. The length and width of the medium are $L$ and $W$ as shown in Fig. 3.1.

\subsubsection{Governing equations}

The flow is governed by the equations for conservation of mass, conservation of momentum in the form of Darcy's law and volume averaged mass balance equation. A convection diffusion equation with a first order irreversible kinetic deposition term is used to describe the transport of NPs. 


$$
\begin{gathered}
\nabla \cdot \vec{V}_{D}=0 \\
\overrightarrow{\nabla P}=-\frac{\mu}{K} \vec{V}_{D} \\
\frac{\partial C_{a}}{\partial t}+\frac{1}{\phi}\left(\overrightarrow{V_{D}} \cdot \vec{\nabla}\right) C_{a}=D_{a} \nabla^{2} C_{a} \\
\frac{\partial C_{b}}{\partial t}+\frac{1}{\phi}\left(\overrightarrow{V_{D}} \cdot \vec{\nabla}\right) C_{b}=D_{b} \nabla^{2} C_{b} \\
\frac{\partial C_{n}}{\partial t}+\frac{1}{\phi}\left(\overrightarrow{V_{D}} \cdot \vec{\nabla}\right) C_{n}=D_{n} \nabla^{2} C_{n}-k_{d e p} C_{n}
\end{gathered}
$$

In the above equations, $\vec{V}_{D}(u, v)$ is Darcy's velocity, $P$ the local pressure, $\mu$ the viscosity, $K$ the permeability, $\phi$ the porosity, $C_{a}, C_{b}, C_{n}$ the volume averaged concentration (or the mass fraction) of component $\mathrm{A}, \mathrm{B}$ and NPs respectively, $D_{i}$ the effective diffusity and $k_{d e p}$ represents the deposition rate which can be described with the widely applied colloidal filtration theory (CFT) [67-69]. According to CFT, the deposition rate is proportional to the particles concentration in the base fluid and $k_{d e p}$ is constant through the porous medium. Furthermore, the model does not consider the detachment of the particle from the solid surface. The equations will be first written in terms of the interstitial velocities and then is formulated in a Lagrangian reference frame moving at the velocity $\frac{U}{\phi}\left(\overrightarrow{u^{\prime}}=\vec{u}-\frac{U}{\phi} \vec{i}\right.$ ). Furthermore, the equations are made dimensionless using diffusive scaling. The viscosity of the solvent $\mu_{s}$ and the initial concentration of fluid $\mathrm{A}, C_{a_{0}}$, are used to make the viscosity and concentration dimensionless. It should be mentioned that in this study the permeability $K$ is assumed constant and incorporated in the expression of the viscosity by treating $\frac{\mu}{K}$ as $\mu$ and the ratios of $\mu$ shall be referred to as either viscosity or mobility ratios.

$$
\begin{gathered}
x^{*}=\frac{x}{\frac{D_{a} \phi}{U}}, y^{*}=\frac{y}{\frac{D_{a} \phi}{U}}, t^{*}=\frac{t}{\frac{D_{a} \phi^{2}}{U^{2}}}, u^{*}=\frac{u}{\frac{U}{\phi}}, \\
v^{*}=\frac{v}{\frac{U}{\phi}}, p^{*}=\frac{P}{\mu_{s} D_{a} \phi}, \mu^{*}=\frac{\mu}{\mu_{s}}, C_{i}^{*}=\frac{C_{i}}{C_{a_{0}}}
\end{gathered}
$$

The resulting dimensionless equations are: 


$$
\begin{gathered}
\frac{\partial u^{*}}{\partial x^{*}}+\frac{\partial v^{*}}{\partial y^{*}}=0 \\
\frac{\partial p^{*}}{\partial x^{*}}=-\mu^{*}\left(1+u^{*}\right), \quad \frac{\partial p^{*}}{\partial y^{*}}=-\mu^{*} v^{*} \\
\frac{\partial C_{a}^{*}}{\partial t^{*}}+u^{*} \frac{\partial C_{a}^{*}}{\partial x^{*}}+v^{*} \frac{\partial C_{a}^{*}}{\partial y^{*}}=\frac{\partial^{2} C_{a}^{*}}{\partial x^{* 2}}+\frac{\partial^{2} C_{a}^{*}}{\partial y^{* 2}} \\
\frac{\partial C_{b}^{*}}{\partial t^{*}}+u^{*} \frac{\partial C_{b}^{*}}{\partial x^{*}}+v^{*} \frac{\partial C_{b}^{*}}{\partial y^{*}}=\delta_{b}\left(\frac{\partial^{2} C_{b}^{*}}{\partial x^{* 2}}+\frac{\partial^{2} C_{b}^{*}}{\partial y^{* 2}}\right) \\
\frac{\partial C_{n}^{*}}{\partial t^{*}}+u^{*} \frac{\partial C_{n}^{*}}{\partial x^{*}}+v^{*} \frac{\partial C_{n}^{*}}{\partial y^{*}}=\delta_{n}\left(\frac{\partial^{2} C_{n}^{*}}{\partial x^{* 2}}+\frac{\partial^{2} C_{n}^{*}}{\partial y^{* 2}}\right)-D a_{d e p} C_{n}^{*}
\end{gathered}
$$

where $D a_{d e p}=\frac{k_{d e p} D_{a} \phi^{2}}{U^{2}}$ represents the dimensionless deposition rate. $\delta_{b}$ and $\delta_{n}$ are the dimensionless diffusion rate of fluid B and NPs with respect to the diffusion of the displacing fluid A. Similarly, using the above mentioned dimensionless groups, the domain dimension will vary from $\left(\frac{-P e}{2}, \frac{P e}{2}\right)$ in the $x$ direction and $\left(\frac{-P e}{2 A_{s}}, \frac{P e}{2 A_{s}}\right)$ in the $y$ direction, where $A_{s}=\frac{L}{W}$ is the cell aspect ratio and $P e=\frac{U L}{\phi D_{a}}$ is the Péclet number. Furthermore, the following boundary conditions are adopted:

$$
\begin{gathered}
\left(u^{*}, v^{*}, C_{a}{ }^{*}, C_{b}{ }^{*}\right)\left(\frac{-P e}{2}, y\right)=(0,0,1,0) \\
\left(u^{*}, v^{*}, C_{a}{ }^{*}, C_{b}{ }^{*}\right)\left(\frac{P e}{2}, y\right)=(0,0,0,1) \\
\frac{\partial C_{n}{ }^{*}}{\partial x}\left(\frac{-P e}{2}, y\right)=\frac{\partial C_{n}{ }^{*}}{\partial x}\left(\frac{P e}{2}, y\right)=0 \\
\left(u^{*}, v^{*}, C_{a}{ }^{*}, C_{b}{ }^{*}, C_{n}{ }^{*}\right)\left(x, \frac{-P e}{2 A_{s}}\right)=\left(u^{*}, v^{*}, C_{a}{ }^{*}, C_{b}{ }^{*}, C_{n}{ }^{*}\right)\left(x, \frac{P e}{2 A_{s}}\right)
\end{gathered}
$$

Eq. 3.8a and 3.8b indicate that fluid $\mathrm{A}$ is always present at the inlet (left boundary) with its initial concentrations, while this applies for fluid B at the outlet (right boundary). It is also assumed that the initial concentrations of fluid A and fluid B are equal. As a result of the deposition, the NPs concentration does not remain constant at the domain inlet and decreases with time to less than $C_{n 0}$, its initial dimensionless concentration. Accordingly, the Neumann boundary condition is implemented for the NP concentration. Furthermore, 
similar to previous studies [25, 70], periodic boundary conditions are used in the transverse direction. In all that follows, the analysis will be conducted with the dimensionless variables from which the asterisks are dropped for convenience. In particular, all results will be presented at dimensionless times.

To complete the model, a form for the dependence of viscosity on concentration must be specified. Here the exponential viscosity-concentration relationship is chosen to complete the model as below [22]:

$$
\mu=\exp \left(R_{a} C_{a}+R_{b} C_{b}+R_{n} C_{n}\right)
$$

where $R_{a}, R_{b}, R_{n}$ are $\log$ mobility ratios defined as follows:

$$
R_{a}=\ln \left(\frac{\mu_{a_{0}}}{\mu_{s}}\right), \quad R_{b}=\ln \left(\frac{\mu_{b_{0}}}{\mu_{s}}\right), \quad R_{n}=\ln \left(\frac{\mu_{n_{0}}}{\mu_{s}}\right)
$$

By considering equal diffusion rates $\left(\delta_{i}=1\right)$ and in the absence of NP deposition ( $\left.D a_{\text {dep }}=0\right)$, one expects the flow to be unstable if $R_{a}+R_{n}<R_{b}$. In the following, the flow instability will be analyzed through LSA and NLS. First the LSA formulation is presented. The linearized equations will be solved analytically and general conclusions will be derived from a long wave and cut-off wave number analysis followed by the full numerical solution. Finally, by NLS of Eq. 3.7 the finger growth and its configuration will be investigated.

\subsection{Linear stability analysis}

\subsubsection{Problem formulation}

After getting the linearized disturbed equations, the pressure $P^{\prime}$ and transverse velocity $v^{\prime}$ disturbances are eliminated by taking the curl of Darcy's law and utilizing the continuity equation (see Appendix A). Since the base state is a function of only the $x$ co-ordinate and time, the disturbances are decomposed in term of Fourier components in the $y$ direction to 
conduct a normal mode analysis:

$$
\left(u^{\prime}, C_{a}^{\prime}, C_{b}^{\prime}, C_{n}^{\prime}\right)(x, y, t)=(\Upsilon, \Phi, X, \Psi)(x, t) \exp (i k y)
$$

Where $k$ is the wave number of disturbances. This leads to the following set of coupled partial differential equations:

$$
\begin{gathered}
\left(\frac{\partial}{\partial t}-\frac{\partial^{2}}{\partial x^{2}}+k^{2}\right) \Phi(x, t)=-\frac{\partial \bar{C}_{a}}{\partial x} \Upsilon(x, y) \\
\left(\frac{\partial}{\partial t}-\delta_{b} \frac{\partial^{2}}{\partial x^{2}}+\delta_{b} k^{2}\right) X(x, t)=-\frac{\partial \bar{C}_{b}}{\partial x} \Upsilon(x, y) \\
\left(\frac{\partial}{\partial t}-\delta_{n} \frac{\partial^{2}}{\partial x^{2}}+\delta_{n} k^{2}+D a_{d e p}\right) \Psi(x, t)=-\frac{\partial \bar{C}_{n}}{\partial x} \Upsilon(x, y) \\
\left(\frac{\partial^{2}}{\partial x^{2}}-k^{2}+\left(R_{a} \frac{\partial \bar{C}_{a}}{\partial x}+R_{b} \frac{\partial \bar{C}_{b}}{\partial x}+R_{n} \frac{\partial \bar{C}_{n}}{\partial x}\right) \frac{\partial}{\partial x}\right) \Upsilon(x, t)=k^{2}\left(R_{a} \Phi+R_{b} X+R_{n} \Psi\right)
\end{gathered}
$$

In a moving reference frame and in the absence of any disturbances, the base state velocity and pressure are defined as $\bar{u}(x, t)=\bar{v}(x, t)=0$ and $\bar{P}(x, t)=-\int^{x} \bar{\mu}\left(x^{\prime}, t\right) d x^{\prime}$. Furthermore, the base state concentration profiles which are derived after solving the one dimensional diffusion equation for fluid $\mathrm{A}$ and $\mathrm{B}$ and the one dimensional diffusion equation with deposition for NPs are:

$$
\begin{gathered}
\bar{C}_{a}(x, t)=\frac{1}{2} \operatorname{erfc}\left(\frac{x}{2 \sqrt{t}}\right) \\
\bar{C}_{b}(x, t)=\frac{1}{2} \operatorname{erfc}\left(\frac{-x}{2 \sqrt{\delta_{b} t}}\right) \\
\bar{C}_{n}(x, t)=\frac{C_{n 0}}{2} \exp \left(-D a_{d e p} t\right) \operatorname{erfc}\left(\frac{x}{2 \sqrt{\delta_{n} t}}\right)
\end{gathered}
$$

The above base state equations are different from the ones reported by Ghesmat et al. [63] as they have defined the fluids front at the entrance of the medium. The $P e$ number is taken large enough to reach constant values for the base state concentrations at both ends of the domain. In order to solve Eq. 3.12 numerically, the quasi steady state approximation (QSSA) is adopted [22]. QSSA is based on the assumption that the growth rate of perturbations 
is faster than the rate of change of the base state, which therefore can be frozen at a time $t_{0}$. Though it has some limitations that have been recognized [71], QSSA has proven to be a very powerful tool to analyze the stability of this system. In particular, it has been successfully used in the stability analysis of systems containing ethylene glycol and methanol (EG-MeOH)-water [72], propylene glycol (PPG)-water [73] and $\mathrm{CO}_{2}$-water [74]. Applying QSSA, the perturbation function of Eq. 3.11 is expressed as:

$$
(\Upsilon, \Phi, X, \Psi)(x, t)=(Y, \phi, \chi, \psi)\left(x, t_{0}\right) \exp (\gamma t)
$$

Where, $\gamma$ is the quasi-static growth rate. Substituting the above expression into Eq. 3.12, the following system of equations is derived:

$$
\begin{gathered}
\left(\gamma-D^{2}+k^{2}\right) \phi=-\frac{d \bar{C}_{a}}{d x} Y \\
\left(\gamma-\delta_{b} D^{2}+\delta_{b} k^{2}\right) \chi=-\frac{d \bar{C}_{b}}{d x} Y \\
\left(\gamma-\delta_{n} D^{2}+\delta_{n} k^{2}+D a_{d e p}\right) \psi=-\frac{d \bar{C}_{n}}{d x} Y \\
\left(D^{2}-k^{2}+\left(R_{a} \frac{\partial \bar{C}_{a}}{d x}+R_{b} \frac{\partial \bar{C}_{b}}{d x}+R_{n} \frac{\partial \bar{C}_{n}}{d x}\right) D\right) Y=k^{2}\left(R_{a} \phi+R_{b} \chi+R_{n} \psi\right)
\end{gathered}
$$

Eq. 3.15 is an eigenvalue problem with boundary conditions $(Y, \phi, \chi, \psi) \rightarrow(0,0,0,0)$ as $x \rightarrow \pm \infty$. It should be mentioned that the domain varies from $\left[-\frac{P e}{2}, \frac{P e}{2}\right]$. However, the LSA is conducted for an infinite Péclet number that results in the most unstable scenario. The quasi static growth rate $\gamma$ is a function of the parameter $t_{0}$, wave number $k$ and the physical properties of the system. It is worth mentioning that this is a temporal stability analysis where the wave number is a real positive number, while the growth rate can be complex. Eq. 3.15 is a system of coupled second order ordinary differential equations with known boundary conditions where $D$ and $D^{2}$ refer to the first and second order derivatives with respect to $x$, respectively. In the following, both analytical and numerical methods are presented to solve the above equations. 


\subsubsection{Analytical solution}

Eq. 3.15 maybe solved analytically if the perturbations were induced to the base state at $t_{0}=0$, where there is no time for diffusion. This implies that the base state is frozen at $t_{0}=0$ with the concentrations in the form of Heaviside step functions. Generally, the sharp front represents the most dangerous situation in terms of instability but it is not always true as discussed in Ref [32]. The resulting characteristic equation for such conditions is (see Appendix B for detailed derivation):

$$
\begin{aligned}
& k \sqrt{\delta_{n} k^{2}+\gamma+D a_{d e p}} \sqrt{\delta_{b} k^{2}+\gamma}\left(\frac{k^{2} R_{a}}{\gamma}-2 \sqrt{k^{2}+\gamma}-\frac{k R_{a}}{\gamma} \sqrt{k^{2}+\gamma}\right) \\
& +\frac{k^{2} C_{n 0} R_{n}}{\gamma+D a_{d e p}} \sqrt{k^{2}+\gamma} \sqrt{\delta_{b} k^{2}+\gamma}\left(k \sqrt{\delta_{n}}-\sqrt{\delta_{n} k^{2}+\gamma+D a_{d e p}}\right) \\
& +\sqrt{\delta_{n} k^{2}+\gamma+D a_{d e p}} \sqrt{k^{2}+\gamma} \frac{k^{2} R_{b}}{\gamma}\left(\sqrt{\delta_{b} k^{2}+\gamma}-k \sqrt{\delta_{b}}\right)=0
\end{aligned}
$$

It is worth noting that $C_{n 0}$ and $R_{n}$ do not appear independently but as a group, hence, Eq. 3.16 does not change if one treats $C_{n 0} R_{n}$ as an independent variable or if simply one sets $C_{n 0}=1$. This can be also understood easily by substituting $\Psi=C_{n 0} \Psi^{*}$ in Eq. B.1-B.4. Accordingly, henceforth $R_{n}$ is used as a representative of $C_{n 0} R_{n}$ in the analytical solution. It is not possible to solve the above algebraic equation analytically. But as shall be seen later, it can be used to get some general conclusions regarding the contribution of long wave disturbances as well as the cut-off wave numbers. Before doing so, some limiting cases are examined to check the validity of the above equation.

By substituting $\delta_{b}=1, \delta_{n}=1$ and $D a_{d e p}=0$ into Eq. 3.16 and after some algebra one gets:

$$
4 \gamma^{2}+4 k(k+R) \gamma+R k^{2}(R+4 k)=0
$$

Where $R=R_{a}+R_{n}-R_{b}$. Solving this equation leads to

$$
\gamma=\frac{-k(k-R)-\sqrt{k^{3}(k+2 R)}}{2}
$$


which is identical to the equation Tan and Homsy [22] reported for a simple binary system. Furthermore, by substituting $D a_{d e p}=0, R_{n}=0$ and $\delta_{n}=1$ into Eq. 3.16, one can reach the following equation reported by Mishra et al. [4] for a binary system with different diffusion rates:

$$
\frac{2 \gamma}{k}=R_{b}\left(1-\frac{k}{\sqrt{k^{2}+\frac{\gamma}{\delta_{b}}}}\right)-R_{a}\left(1-\frac{k}{\sqrt{k^{2}+\gamma}}\right)
$$

It should be noted that in the absence of NPs, a binary (A-B) system will be intrinsically unstable if the displaced fluid is more viscous than the displacing one $\left(R_{b}>R_{a}\right)$ or if the displaced fluid is less viscous than the displaced one $\left(R_{b}<R_{a}\right)$ and the two fluids diffuse at

different rates with $\delta_{b}<\left(\frac{R_{b}}{R_{a}}\right)^{\frac{2}{3}}$. This condition is also valid for $R_{b}=R_{a}>0$. Otherwise, displacement will be intrinsically stable [4]. In what follows long wave disturbances asymptotic expansions are presented followed by an analysis of the cut-off wave number. The results are presented for intrinsically unstable displacements followed by a stable ones. This will lead to interesting results without solving the equations directly.

\section{Intrinsically unstable displacements}

Long wave expansion analysis is a very useful tool to predict the instability condition for very small wave numbers. By assuming $\gamma=\Lambda k^{m}$, Eq. 3.16 results in series expansion with respect to $k$ as $k \rightarrow 0$. The following first order expansion is obtained:

$$
\gamma=\frac{R_{b}-R_{a}}{2} k+o(k)
$$

Eq. 3.20 indicates that there is a long wave instability in the system for $R_{b}>R_{a}$. Further analysis for the special case of $R_{b}=R_{a}$ and $\delta_{b} \neq 1$ results in the following expression:

$$
\gamma=\left(\frac{R_{a}\left(1-\sqrt{\delta_{b}}\right)}{2}\right)^{\frac{2}{3}} k^{\frac{4}{3}}+o\left(k^{\frac{4}{3}}\right)
$$

Eq. 3.21 is valid when $R_{a}\left(1-\sqrt{\delta_{b}}\right)>0$. Accordingly, for a positive $R_{a}$ the system is 
unstable for long wave disturbances if $\delta_{b}<1$ and vice versa. The above results show that the highest order term in the long wave expansions does not involve any of the properties of the nanofluid. This implies that NPs have no effect on the onset of long wave instabilities and the problem reduces to that of a binary system with double diffusion effects. Let's now examine the cut-off wave number $k_{c}$, defined as the largest wave number that makes the flow unstable. Using the limit $\gamma \rightarrow 0$ in Eq. 3.16 leads to the following algebraic equation for determining $k_{c}$ :

$$
\Gamma(k)=\frac{R_{b}}{2 \delta_{b}}-\frac{R_{a}}{2}-\frac{R_{n}}{D a_{d e p}}\left(k^{2}-\frac{k^{3} \sqrt{\delta_{n}}}{\sqrt{\delta_{n} k^{2}+D a_{d e p}}}\right)-2 k=0
$$

The largest real root of the above equation gives the cut-off wave number beyond which the flow is stable. Eq. 3.22 indicates that unlike the long wave expansion, the properties of the NPs have effects on the cut-off wave number. It is not possible to solve the above equation analytically, however interesting conclusions may be drawn as shall be seen later. The aim here is to predict the nature of the dispersion curves for the variation of $\Re(\gamma)$ with the wave number $\mathrm{k}$, using both long wave and cut-off wave number analyses. As discussed by Cross and Hohenberg [75] there are three types of dispersion curves. The first type of dispersion curves (Type I) cross the line $\Re(\gamma)=0$ at three different wave numbers $k=0$, $k_{1}$ and $k_{2}$ with the flow being unstable for $k_{1}<k<k_{2}$. However the flow is stable for $0<k<k_{1}$ and $k>k_{2}$. Type II curves cross the line $\Re(\gamma)=0$ at two different wave numbers; $k=0$ and $k_{c}$ such that the value of $\Re(\gamma)$ is positive at $0<k<k_{c}$ and the flow is unstable for all the range of wave numbers less than $k_{c}$. The third type of dispersion curve in which $\Re(\gamma) \neq 0$ at $k=0$ is not possible in our case.

As discussed above, the system is unstable for long waves when $R_{b}>R_{a}$ or $R_{b}=R_{a}>0$, $\delta_{b}<1$ or $R_{b}=R_{a}<0, \delta_{b}>1$. Accordingly, it is concluded that the dispersion curve is type II in these conditions. Further analysis is needed for the case of $R_{b}<R_{a}$ to conclude whether the system is intrinsically unstable. Since $\lim _{k \rightarrow \infty} \Gamma(k)=-\infty$, if $\Gamma(0)>0$ there must 
be at least one root in $(0, \infty)$. Accordingly, the flow is unstable for $R_{b}<R_{a}$ and $\delta_{b}<\frac{R_{b}}{R_{a}}$. This indicates that the conclusion derived for the case of $R_{b}=R_{a}$ above is a special case of this situation. These conclusions are consistent with the results of Mishra et al. [4] for binary systems at $t_{0}=0$ which should be compatible with our results for long waves. The analysis could not predict the type of characteristic curve for this case but the numerical solution of Eq.3.19 for long waves shows that it must be type II. On the other hand for the case of $R_{b}<R_{a}$ and $\delta_{b}>\frac{R_{b}}{R_{a}}, \Gamma(k)$ will be always negative and the flow is stable except for $R_{n}<0$. In the latter case $\Gamma(k)$ may have roots and the system will be unstable.

To summarize, the flow is unstable for long waves when $R_{b}>R_{a}$ regardless of the values of other properties and the characteristic curve is type II. Furthermore, in case of $R_{b}=R_{a}>0\left(R_{b}=R_{a}<0\right)$ the flow is unstable as long as $\delta_{b}<1\left(\delta_{b}>1\right)$ and the characteristic curve is again type II. In addition, the system is unstable with the characteristic

curve of type II in the case of $R_{b}<R_{a}, \delta_{b}<\frac{R_{b}}{R_{a}}$. This analysis shows that the properties of the fluids have a critical role with respect to the properties of NPs in the stability of the system. In other words it could be concluded that when a binary system is unstable, addition of NPs cannot make it stable and the instabilities grow at least for long waves. But what about the opposite scenario? Do they make the stable system unstable? In the next part the instability analysis will be conducted for the critical stable case of $R_{b}=R_{a}, \delta_{b}=1$.

\section{Intrinsically Stable Displacements}

The system without NPs is stable when $R_{b}<\operatorname{Min}\left(R_{a}, \delta_{b} R_{a}\right)$. Analyzing the critical case of $R_{b}=R_{a}, \delta_{b}=1$ would help understand the possible effects of NPs on the stability of the system. In this case the characteristic equation (Eq. 3.16) reduces to:

$$
\left(k^{2}+\gamma\right)\left\{-2 \sqrt{\delta_{n} k^{2}+\gamma+D a_{d e p}}+\frac{k R_{n}}{\gamma+D a_{d e p}}\left(k \sqrt{\delta_{n}}-\sqrt{\delta_{n} k^{2}+\gamma+D a_{d e p}}\right)\right\}=0
$$

By solving this equation it is concluded that for $R_{n}>0$ the flow is stable (See Ap- 
pendix C). In the case of $R_{n}<0$, the flow can be stable or unstable depending on the rate of the NPs diffusion and deposition. In particular, the system is unstable if $R_{n}<$ $-\sqrt{4(5.5+2.5 \sqrt{5}) \delta_{n} D a_{d e p}}$ (See Appendix C) and stable otherwise. It should be mentioned that in most cases, the addition of NPs would increase the viscosity of the base fluid i.e. $R_{n}>0$. There are however cases that show the opposite trend leading to negative values of $R_{n}$ [76]. The characteristic curve in this case is type I and consists of two separate equations as below (See Appendix C). Without this analytical solution one could have also predicted from the long wave analysis that where NPs can destabilize a flow, must have the characteristic curve of type I:

$$
\begin{gathered}
\gamma=-k^{2}, \quad k<k_{c r} \\
\gamma=-\frac{1}{2} \delta_{n} k^{2}-\frac{1}{2} k R_{n}-D a_{d e p}-\frac{k}{2} \sqrt{\delta_{n}^{2} k^{2}-2 \delta_{n} R_{n} k}, \quad k>k_{c r}
\end{gathered}
$$

Where $k_{c r}$ is the wave number in which the characteristic curve equation changes form. Equating the two separate equations in Eq. 3.24 leads to the following equation to find $k_{c r}$ (See Appendix C)

$$
4\left(1-\delta_{n}\right) k_{c r}^{4}-4\left(1-\delta_{n}\right) R_{n} k_{c r}^{3}+\left(R_{n}^{2}+4\left(\delta_{n}-2\right) D a_{d e p}\right) k_{c r}^{2}+4 R_{n} D a_{d e p} k_{c r}+4\left(D a_{d e p}\right)^{2}=0
$$

$k_{c r}$ has the following criteria:

1. $\left(2-\delta_{n}\right) k_{c r}{ }^{2}-R_{n} k_{c r}-2 D a_{d e p} \geq 0$

2. If after considering the first condition more than one positive real root is left, the smaller root is $k_{c r}$.

To validate the results, Eq. 3.23 is solved numerically for $\delta_{n}=1$ and $D a_{\text {dep }}=0.01$ for different $R_{n}$ and the variation of $\operatorname{Max}(\gamma)$ with respect to the wave number is illustrated along with the characteristic curves of Eq. 3.24. According to the above mentioned expression it is predicted that the system is unstable for $R_{n}<-0.67$. As shown in Fig.2 the results are 


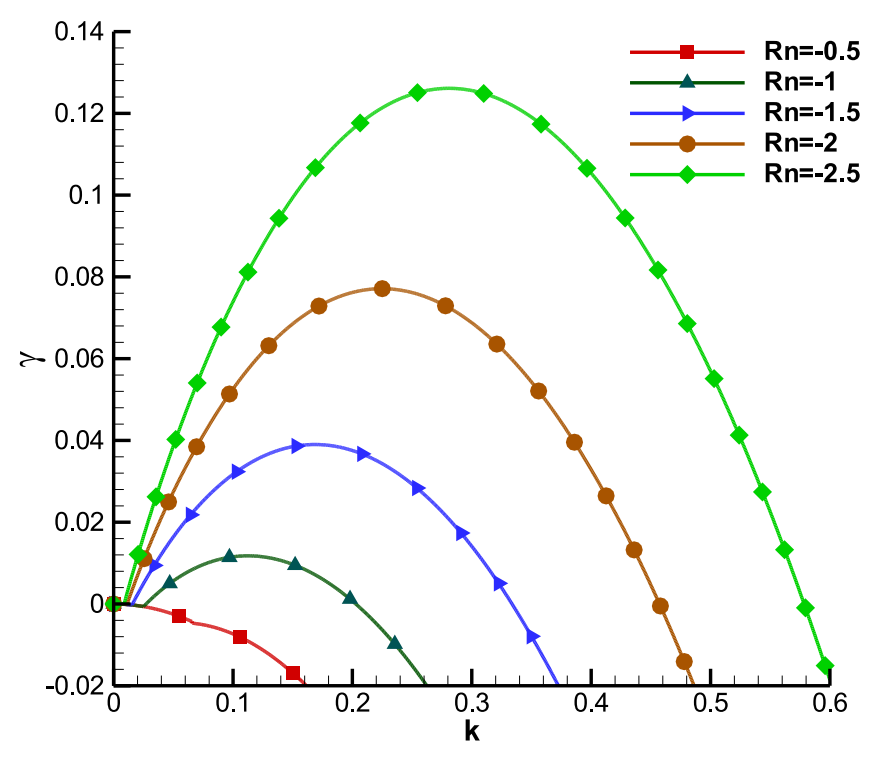

Figure 3.2: Characteristic curve for $R_{a}=R_{b}, \delta_{b}=1, \delta_{n}=1, D a_{d e p}=0.01$ and different $R_{n}$. Lines represent the results of Eq. 3.24 and symbols represent the numerical solution of Eq. 3.23

compatible with each other. The figure shows that with $R_{n}=-0.5, \gamma<0$ in response to the perturbations of different wave numbers and so the flow is stable. It should be mentioned that the characteristic curve of Eq. 3.25 is just valid for $R_{n}<0$.

Finally, to answer the question in the last section, it can be said that when a binary system is stable, the NPs cannot make the system unstable for the common positive values of $R_{n}$.

\subsubsection{Numerical results at $t_{0}=1$}

The above analysis shows that for $R_{b}>R_{a}$, the flow is unstable to long wave disturbances and the characteristic curve is of type II which is in contrast with the numerical results of Ghesmat et. al [63] who have reported a stable system for long waves. In order to validate our conclusions and also for investigating other scenarios, the system of eigenvalue ODEs of Eq. 3.15 is solved numerically. Furthermore, the results derived from LSA will be used to validate the NLS which will be presented in the next section. Using second order central discretization with respect to $x$ with non-uniform grid points and taking advantage of the 
boundary conditions the following system of equations is obtained:

$$
\begin{gathered}
(\underline{A}-\gamma \underline{I}) \phi=\underline{C_{a}} Y \\
(\underline{B}-\gamma \underline{I}) \chi=\underline{C_{b}} Y \\
(\underline{C}-\gamma \underline{I}) \psi=\underline{C_{n}} Y \\
\underline{D} Y=k^{2}\left(\underline{R_{a}} \phi+\underline{R_{b}} \chi+\underline{R_{n}} \psi\right)
\end{gathered}
$$

$\underline{A}, \underline{B}, \underline{C}$ and $\underline{D}$ are tridiagonal matrices while $\underline{C_{a}}, \underline{C_{b}}, \underline{C_{n}}, \underline{R_{a}}, \underline{R_{b}}$ and $\underline{R_{n}}$ are diagonal matrices. By eliminating $Y$ the final equation is written in the following form.

$$
\left[\begin{array}{ccc}
\underline{A}-k^{2} \underline{R_{a} C_{a} D^{-1}} & -k^{2} \underline{R_{b} C_{a} D^{-1}} & -k^{2} \underline{R_{n} C_{a} D^{-1}} \\
-k^{2} \underline{R_{a} C_{b} D^{-1}} & \underline{B}-k^{2} \underline{R_{b} C_{b} D^{-1}} & -k^{2} \underline{R_{n} C_{b} D^{-1}} \\
-k^{2} \underline{R_{a} C_{n} D^{-1}} & -k^{2} \underline{R_{b} C_{n} D^{-1}} & \underline{C}-k^{2} \underline{R_{n} C_{n} D^{-1}}
\end{array}\right]\left[\begin{array}{l}
\phi \\
\chi \\
\psi
\end{array}\right]=\gamma\left[\begin{array}{l}
\phi \\
\chi \\
\psi
\end{array}\right]
$$

The goal in QSSA is to find the eigenvalues $(\gamma)$ with the largest real part corresponding to each wave number. It is also worth mentioning that the length of the domain $(P e)$ was chosen long enough to ensure the eigenfunctions decay at both ends of the domain according to the boundary conditions. In what follows the viscosities of the solutions are chosen to represent an unstable condition, and the results are presented and discussed for $R_{a}=2$, $R_{b}=5$. Furthermore, it is assumed that the presence of NPs increases the viscosity of the base state $\left(R_{n}>0\right)$. For convenience the initial concentration of the NPs is assumed to be equal to one $\left(C_{n 0}=1\right)$. Results of the numerical solutions which for brevity are not presented here, show that the increases of $R_{n}$ and $D a_{d e p}$ have stabilizing and destabilizing effects, respectively as discussed by Ghesmat et al. [63], but in contrast with their results, the characteristic curves are type II in concordance with the analytical solution. Furthermore, the results confirm the conclusion of the analytic solution indicating that the presence of NPs has virtually no effect on the long wave disturbances (see Fig. 3.3). Fig. 3.3 shows that the presence of NPs makes the system less unstable, but interestingly the system is unstable even at high $R_{n}$ where $R_{a}+R_{n}>R_{b}$. This again validates the results of the analytical solution which indicates that the system is unstable as long as $R_{b}>R_{a}$. This is as a result 
of NP deposition and will be discussed broadly in the next part. Furthermore, comparing Fig. $3.3 \mathrm{a}$ and $3.3 \mathrm{~b}$ it can be seen that the system is less unstable when $\delta_{b}>1$. In this case the large viscosity fluid B diffuses more to the upstream and makes the flow less unstable by attenuating the viscosity contrast. This can lead to the creation of locally stable regions adjacent to the $X=0$ as illustrated by the base state viscosity profile in Fig. 3.4. The sudden change in the slope of the characteristic curves for $\delta_{b}=2$ in Fig. 3.3 is attributed to a change in the order of the imaginary part of $\gamma$ which is as a result of the double diffusion effect that has been discussed in Ref [4] and [43].

(a)

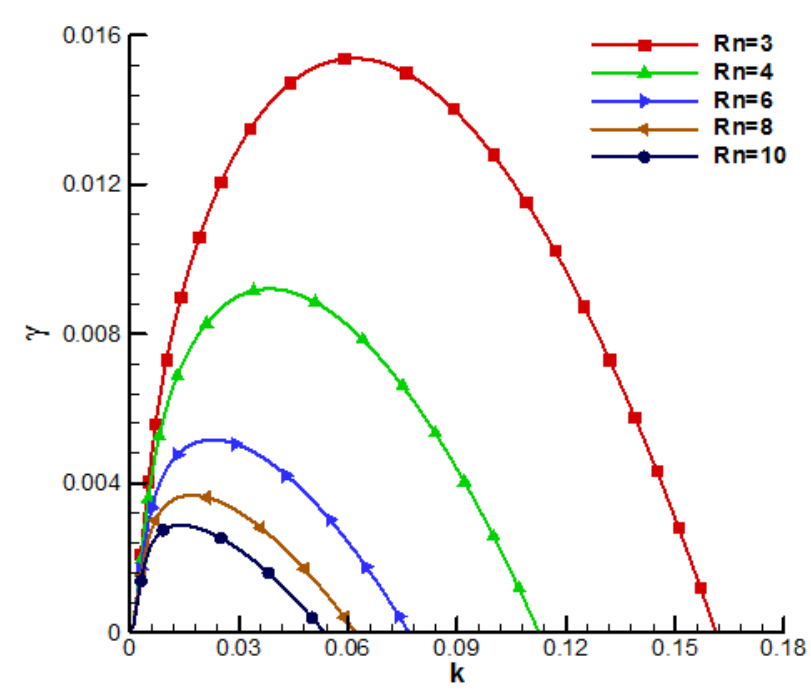

(b)

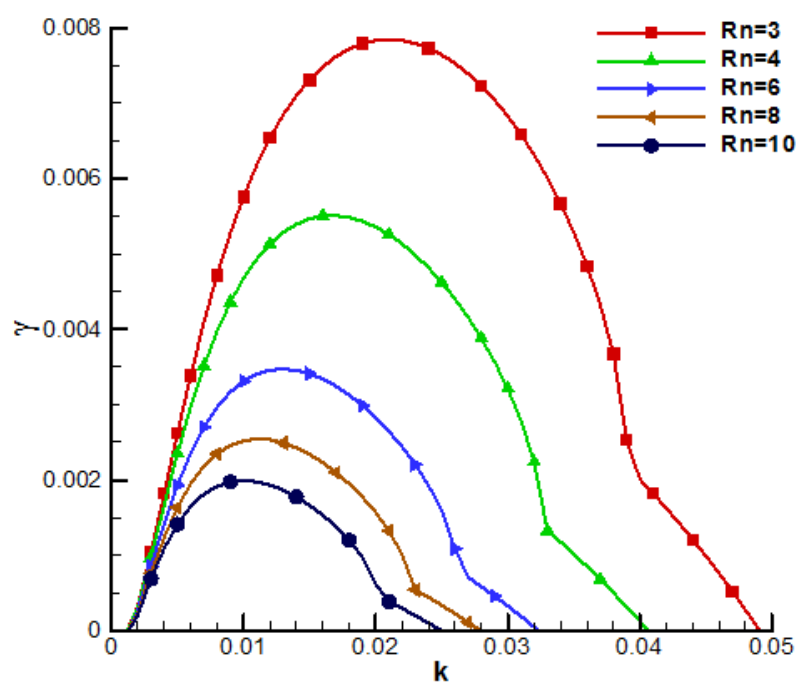

Figure 3.3: Characteristic curves at different nanofluid viscosity, $\delta_{n}=1, D a_{\text {dep }}=0.01$, (a) $\delta_{b}=1$, (b) $\delta_{b}=2$

Fig. 3.5 shows the effect of NP diffusion. An increase in $\delta_{n}$ causes the system to be more unstable which is in contrast to the results of Ref [63] where it was reported that for $\delta_{n}<1$ larger $\delta_{n}$ makes the system less unstable while in case of $\delta_{n}>1$ the opposite trend takes place. One can say that as $\delta_{n}$ increases, NPs diffuse more to the downstream and by increasing the local viscosity creates a more intensified viscosity mismatch in the system.

In order to complete the discussion let's have a look at the characteristic curves of the situation where $R_{b}<R_{a}\left(R_{a}=2, R_{b}=1\right)$ in the presence of NPs with $R_{n}>0$. Fig. 3.6a 


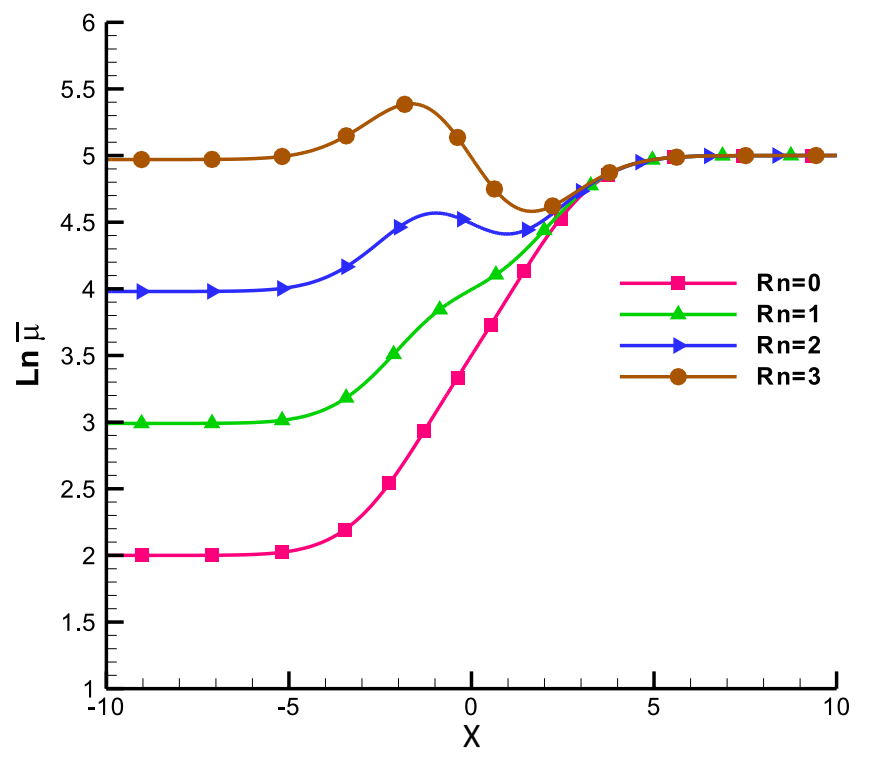

Figure 3.4: Variation of the base state viscosity along the domain at higher $\delta_{b}=2, \delta_{n}=1$, $D a_{\text {dep }}=0.01$

(a)

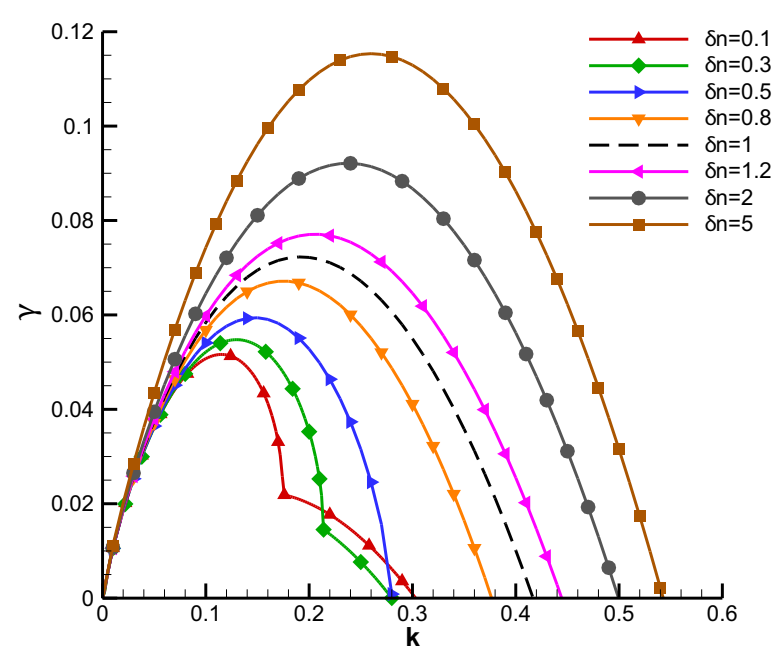

(b)

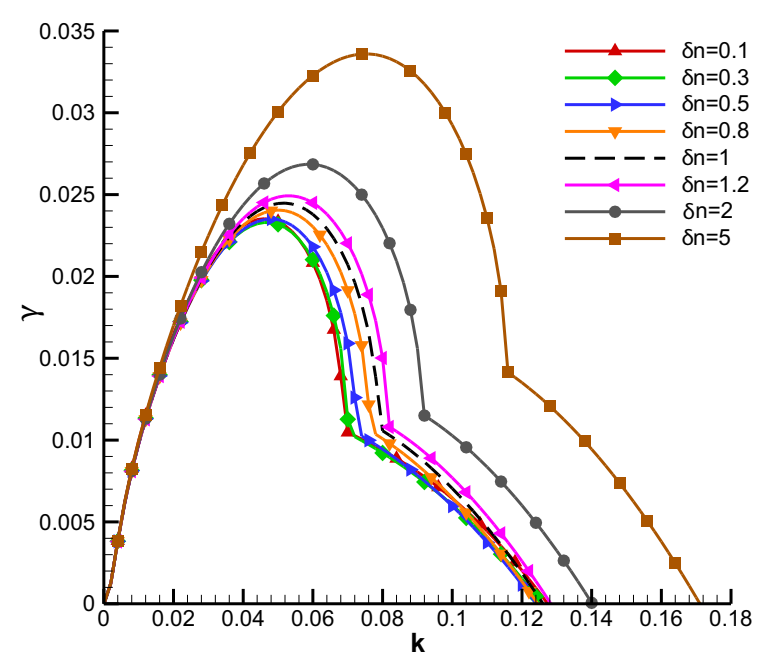

Figure 3.5: Effect of the NP diffusion, $R_{n}=1, D a_{d e p}=0.01$ (a) $\delta_{b}=1$, (b) $\delta_{b}=2$ 
(a)

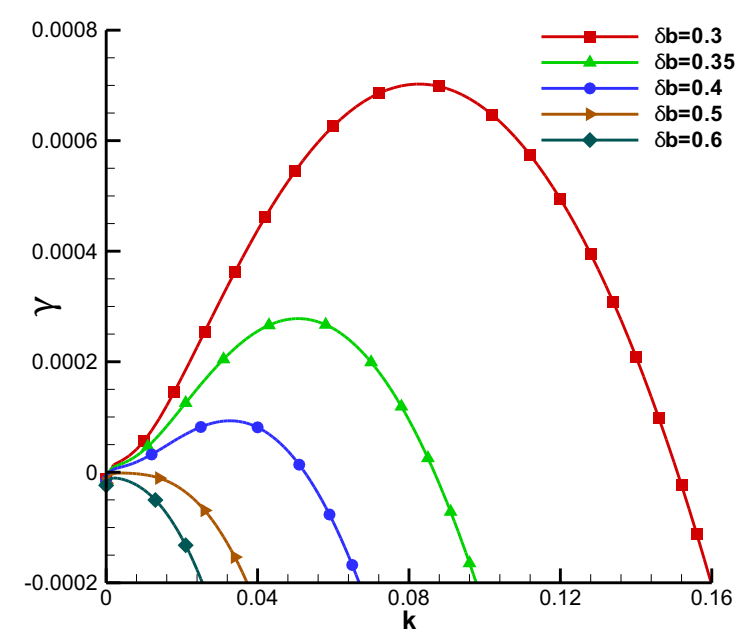

(b)

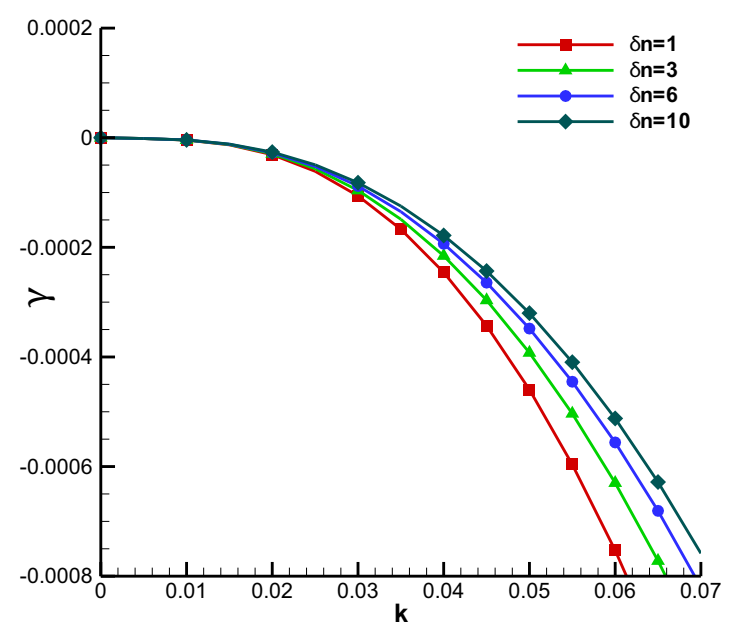

Figure 3.6: Effect of the diffusion of both displaced fluid and NPs when $R_{b}<R_{a}$ and $R_{n}>0$, $R_{a}=2, R_{b}=1, R_{n}=1, D a_{d e p}=0.01$, (a) $\delta_{n}=1$, (b) $\delta_{b}=0.5$

shows that as long as $\delta_{b}<0.5$ the system is unstable which is in concordance with the analytical solution. This indicates that although NPs lead to an increase of the viscosity of the upstream solution, the stability condition still depends on the properties of the fluids. The role of the NPs is just reducing the intensity of the instability by reducing the growth rate with respect to the binary solution. The neutral role of the properties of NPs (where $R_{n}>0$ ) in the case of a stable binary system is well depicted in Fig. 3.6b. Although $\delta_{b}$ is chosen in its critical value $\left(\delta_{b}=\frac{R_{b}}{R_{a}}=0.5\right)$ the diffusion of NPs cannot make the system unstable while as illustrated in Fig. 3.5, they can increase the instability of an unstable system.

\subsection{Non-linear simulations}

\subsubsection{Numerical technique}

Direct simulations of Eq. 3.7 have been conducted to gain insight into the finger growth and study the effects of non-linear terms which were ignored in LSA. A pseudo-spectral Hartley based scheme [30] has been used to solve the equations. By taking the curl of Darcy's 
law, writing velocities in the form of stream functions and taking advantage of Eq. 3.9 for viscosity the final equations are written as a function of vorticity $(\omega)$, stream function $(\psi)$ and concentration $\left(C_{i}\right)$. Then following earlier studies, the model equations are formulated in terms of a base state $\left(\bar{C}_{i}(x, t)\right)$ and a perturbation $\left(C_{i}{ }^{\prime}(x, y, t)\right)$ that decays to zero at the domain's stream-wise boundaries. Such formulation results in periodic conditions and allows the fast Hartley transform to solve the governing equations for the perturbations:

$$
\begin{gathered}
\frac{\partial C_{a}{ }^{\prime}}{\partial t}=J_{a}+\frac{\partial^{2} C_{a}{ }^{\prime}}{\partial x^{2}}+\frac{\partial^{2} C_{a}{ }^{\prime}}{\partial y^{2}} \\
\frac{\partial C_{b}{ }^{\prime}}{\partial t}=J_{b}+\delta_{b}\left(\frac{\partial^{2} C_{b}{ }^{\prime}}{\partial x^{2}}+\frac{\partial^{2} C_{b}{ }^{\prime}}{\partial y^{2}}\right) \\
\frac{\partial C_{n}{ }^{\prime}}{\partial t}=J_{n}+\delta_{n}\left(\frac{\partial^{2} C_{n}{ }^{\prime}}{\partial x^{2}}+\frac{\partial^{2} C_{n}{ }^{\prime}}{\partial y^{2}}\right)-D a_{d e p} C_{n}{ }^{\prime} \\
\omega=R_{a} N_{a}+R_{b} N_{b}+R_{n} N_{n} \\
\nabla^{2} \psi=-\omega
\end{gathered}
$$

Where:

$$
\begin{aligned}
J_{i} & =\frac{\partial \psi}{\partial x} \frac{\partial C_{i}^{\prime}}{\partial y}-\frac{\partial \psi}{\partial y}\left(\frac{\partial \bar{C}_{i}}{\partial x}+\frac{\partial C_{i}^{\prime}}{\partial x}\right) \\
N_{i} & =\frac{\partial \psi}{\partial x}\left(\frac{\partial \bar{C}_{i}}{\partial x}+\frac{\partial C_{i}^{\prime}}{\partial x}\right)+\left(1+\frac{\partial \psi}{\partial y}\right) \frac{\partial C_{i}^{\prime}}{\partial y}
\end{aligned}
$$

$\psi(x, y, t), \omega(x, y, t)$ and $C^{\prime}{ }_{i}(x, y, t)$ are zero at the boundaries. Moreover, a random number distribution of concentrations is used as the initial condition:

$$
c^{\prime}\left(x, y, t_{0}\right)=\delta_{p} \operatorname{rand}(y) \exp \left(-\frac{x^{2}}{\sigma_{p}^{2}}\right)
$$

where $\operatorname{rand}(y)$ is a random number between $(-1,1)$, the perturbation magnitude $\delta_{p}$ is a small number relative to unity and ${\sigma_{p}}^{2}$, also a very small number, represents the penetration of the disturbances in the domain. The resulting ordinary differential equations in the transform space are stepped in time using a semi-implicit algorithm based on the Adams-Bashforth and Adams-Moulton predictor-corrector scheme. See Appendix D for a brief explanation 

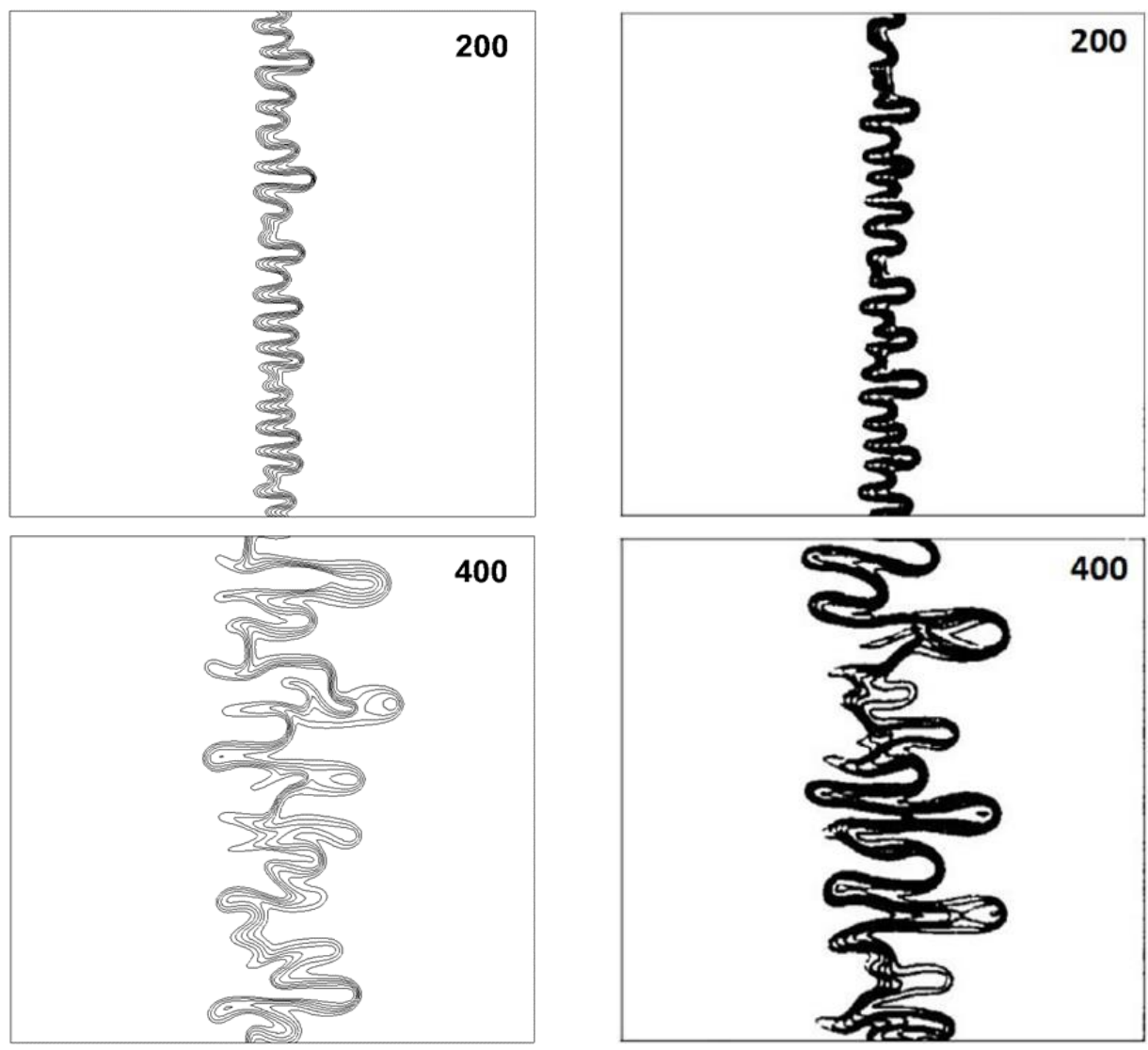

Figure 3.7: Iso-contours of $C_{a}\left(0.1 \leq C_{a} \leq 0.6\right)$ from the present study (left) and Singh [78] (right), $R=3, P e=1000, A_{s}=1$.

of the solution procedure. More details can be found in [30,77]. The code was validated with the results of NLS of a binary system reported in the literature by considering $\delta_{b}=1$, $\delta_{n}=1, D a_{d e p}=0$ and $R=R_{b}-R_{a}-R_{n}$. As shown in Fig.3.7 the finger configurations are qualitatively similar to those reported by Singh [78]. LSA was also used to validate the code by checking that the wave number corresponding to the maximum growth rate corresponds to the wave number of the instabilities at the early stages of the NLS.

In all that follows a cell aspect ratio $A_{s}=2$ and $P e=1024$ is assumed. Results are presented in the form of iso-surface of fluid A, and only time frames that illustrate the most important structures are shown. In addition, plots of the mixing length (ML) are shown to further quantify the instability. The ML is defined as the ratio of the length within concentration range $C_{a, a v}=0.01$ to $C_{a, a v}=0.99$ to the length of the whole domain [25] 

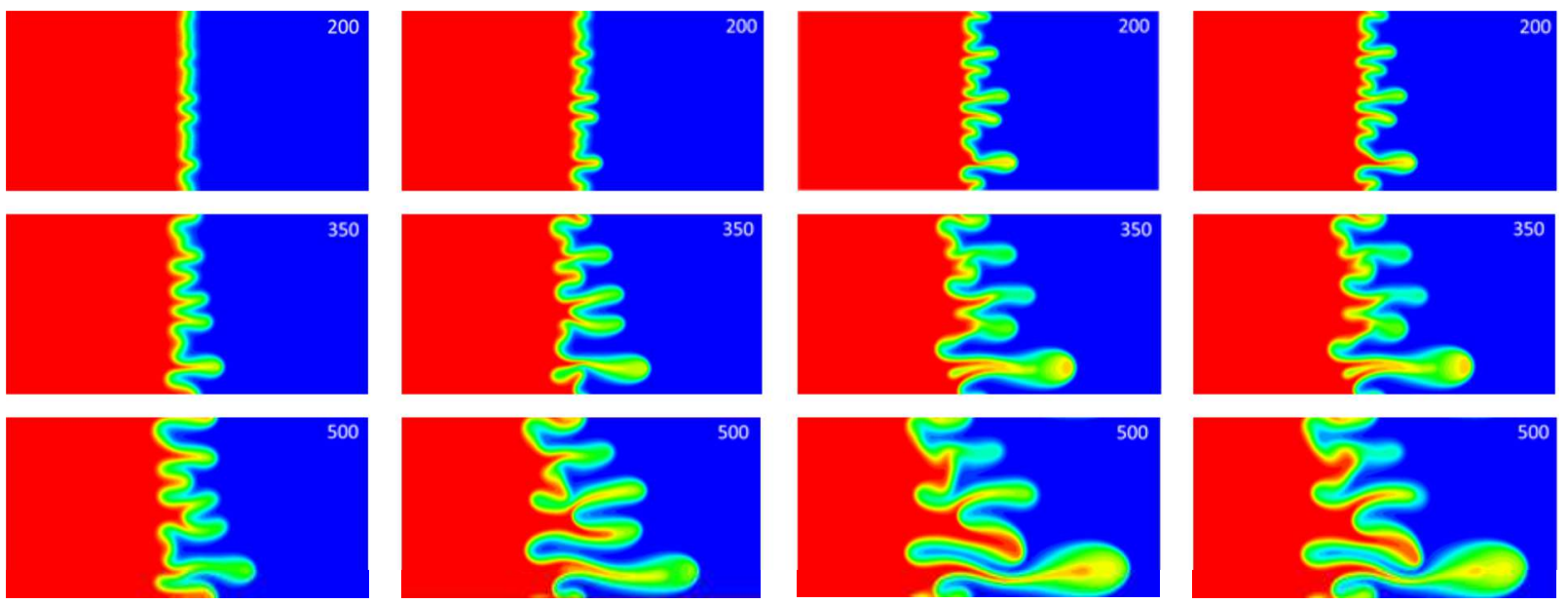

(a) $D a_{d e p}=0$

(b) $D a_{d e p}=0.005$

(c) $D a_{d e p}=0.05$

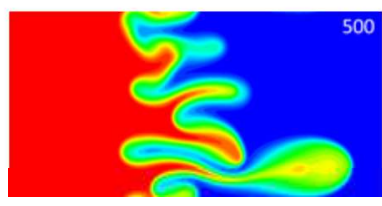

(d) $D a_{d e p}=0.5$

Figure 3.8: Contours of $C_{a}$ at different NP deposition rate, $R_{n}=1, \delta_{b}=1, \delta_{n}=1$

where:

$$
C_{a, a v}(x, t)=\frac{A_{s}}{P e} \int_{0}^{\frac{P e}{A_{s}}} C_{a}(x, y, t) d y
$$

Larger values of ML indicate a more unstable situation and vice versa. Furthermore, wherever it seems helpful, the discussion is extended with the contribution of the concept of breakthrough time, the time for fingers to reach the end of the domain.

\subsubsection{Results and discussion}

The important feature of the presence of NPs is their effect on the viscosity which is interconnected with the effect of the deposition rate. In this stage, the viscosities of the fluids are chosen such that the flow is unstable in the absence of NPs. Accordingly, in all what follows, as a representative of an unstable condition $R_{a}=2, R_{b}=5$ is taken.

Fig. 3.8 shows the development of instabilities for different deposition rates. It is clear that as the deposition rate increases the flow becomes more unstable with more intricate finger structures. These qualitative trends are confirmed with the analysis of the ML where larger deposition rate leads to larger ML and is demonstrated in Fig. 3.9. 


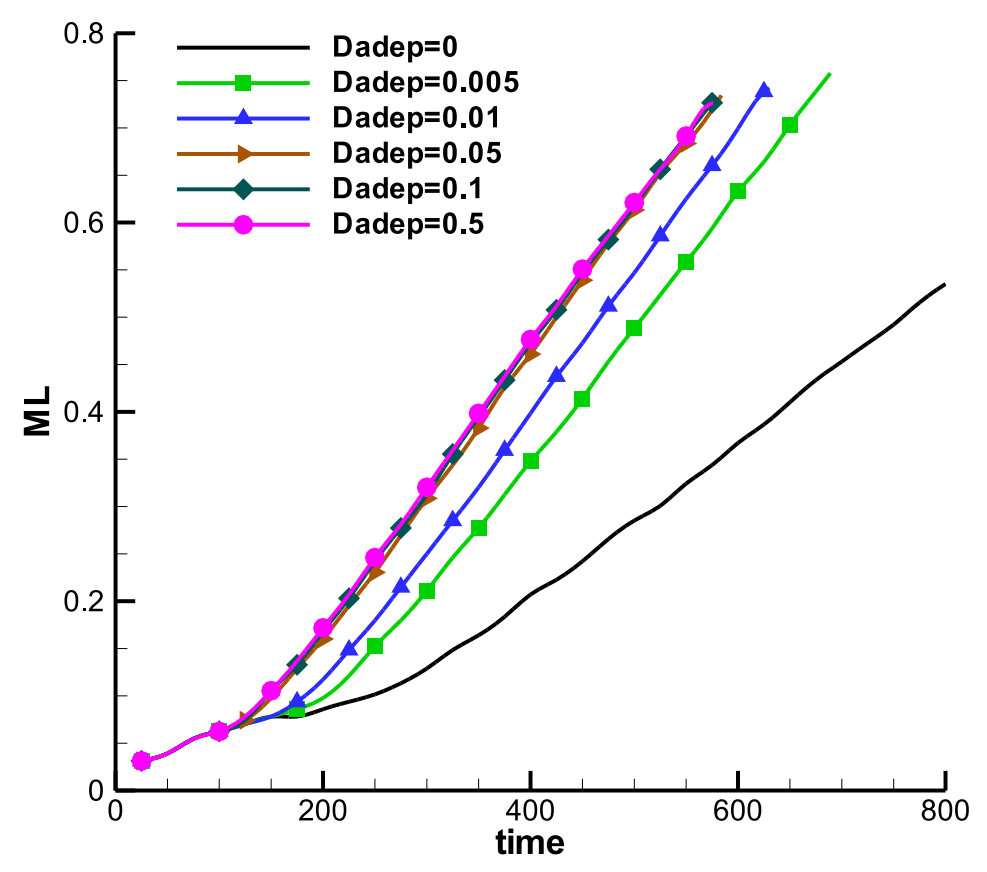

Figure 3.9: Mixing length over time for different deposition rates, $R_{n}=1, \delta_{b}=1, \delta_{n}=1$

This trend can be explained by the fact that an increase in the deposition rates result in a reduction of NPs which in turn affects the viscosity contrast of the system (see contours of $C_{n}$ in Fig. 3.10). However, one should consider that further increase in the deposition rate was found not to change the ML and the finger complication. This can be attributed to the fact that at high deposition rates, NPs are removed rapidly and at some point, are completely eliminated from the system (see Fig. 3.10c). As a result, further increase in the deposition rate will have no effect on the instability.

The presence of deposition leads to a significant effect in the fluid flow system. Considering the system of Eq. 3.28, one can conclude that by neglecting $D a_{d e p}$ and assuming a unit diffusion rate, the system is controlled by an effective viscosity ratio $R=R_{a}+R_{n}-R_{b}$. If $R \geq 0$ the flow is stable and it is unstable otherwise. Fig. 3.11 depicts results of the concentration of fluid A for $R_{n}=5, \delta_{b}=1$ and for two cases with and without NP deposition. Although $R \geq 0$ in both systems, it is clear that deposition has changed the displacement from a stable one (Fig. 3.11a) to an unstable flow with a front exhibiting intricate fingers. This change in the nature of the displacement instability was predicted from the linear stabil- 


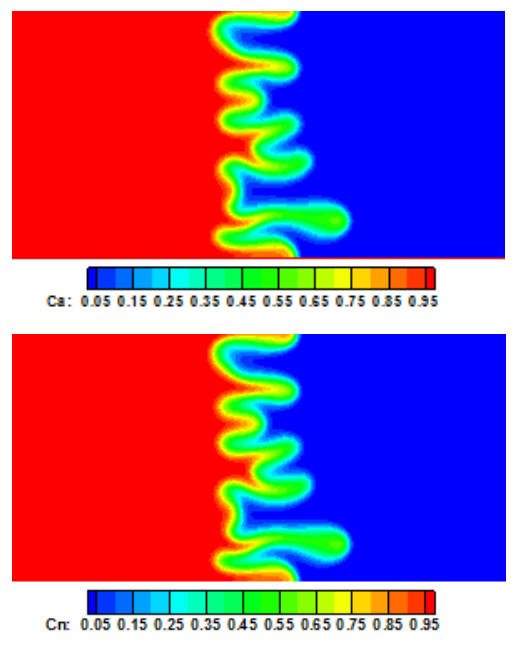

(a) $D a_{d e p}=0$

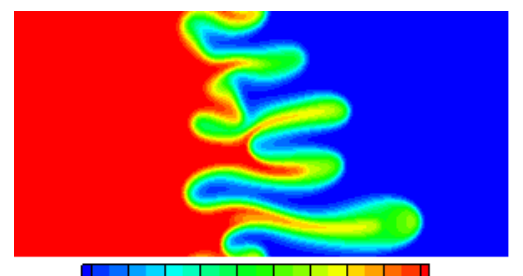

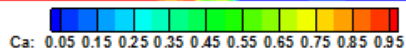

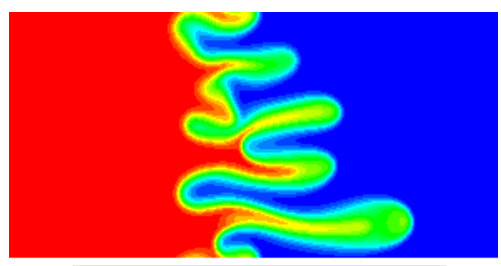

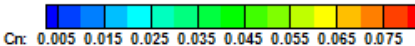

(b) $D a_{\text {dep }}=0.005$

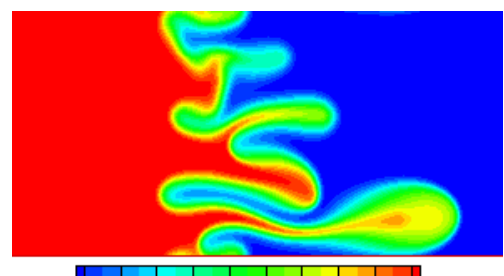

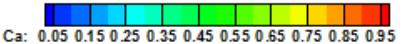

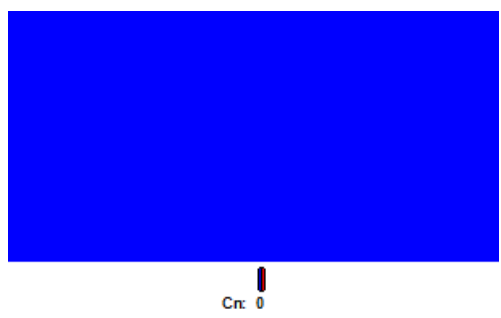

(c) $D a_{d e p}=0.05$

Figure 3.10: Contours of $C_{a}$ and $C_{n}$ at three NP deposition rates at $t=500, R_{n}=1, \delta_{b}=1$, $\delta_{n}=1$. It is seen that at $D a_{d e p}=0.5$ the NPs are completely removed from the system.

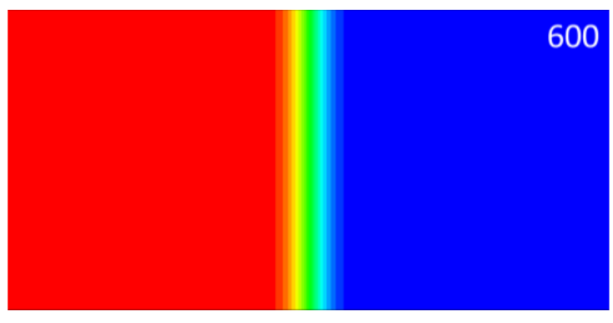

(a) $D a_{d e p}=\mathbf{0}$

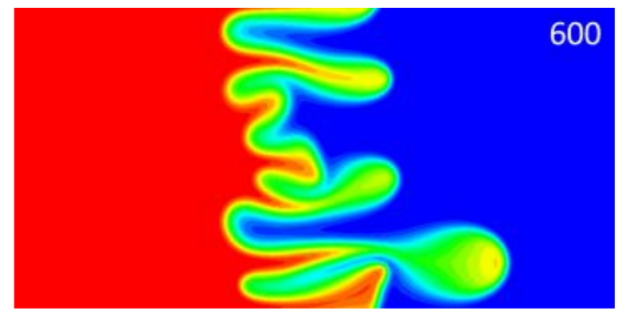

(b) $D a_{d e p}=0.01$

Figure 3.11: Contours of $C_{a}$ representing the role of deposition rate in changing the stability of the system, $R_{n}=5, \delta_{b}=1, \delta_{n}=1$

ity analysis in the previous section, and in particular from the long wave expansion analysis of Eq. 3.20. One may however ask how does the presence of deposition can make the initially stable system (not binary stable system), unstable? Analyzing the variation of the base state viscosity and concentrations of Fig. 3.12 along the channel shows that although the system is stable at early times, as time passes, due to the deposition and the removal of NPs, the viscosity distribution changes from monotonically decreasing to monotonically increasing one, and finally makes the system unstable.

It should be mentioned that despite the presence of deposition, the effect of the initial viscosity ratio of the nanofluids $\left(R_{n}\right)$ in controlling the instabilities is very significant. This 
(a)

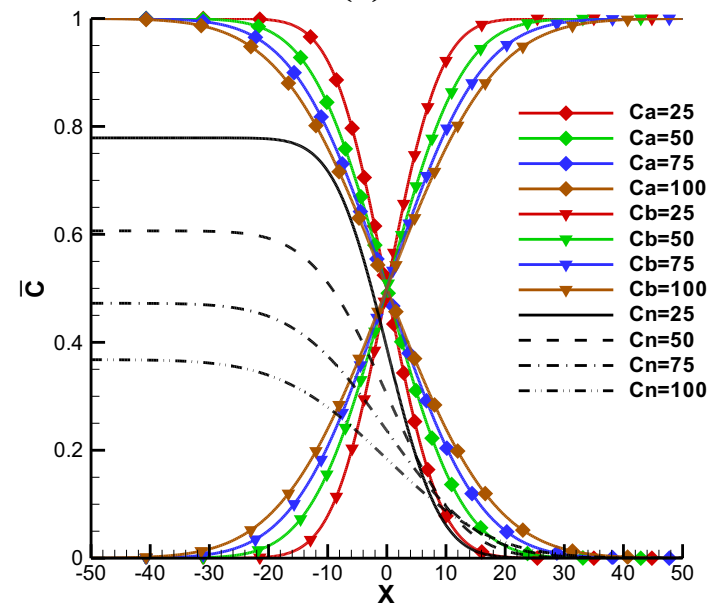

(b)

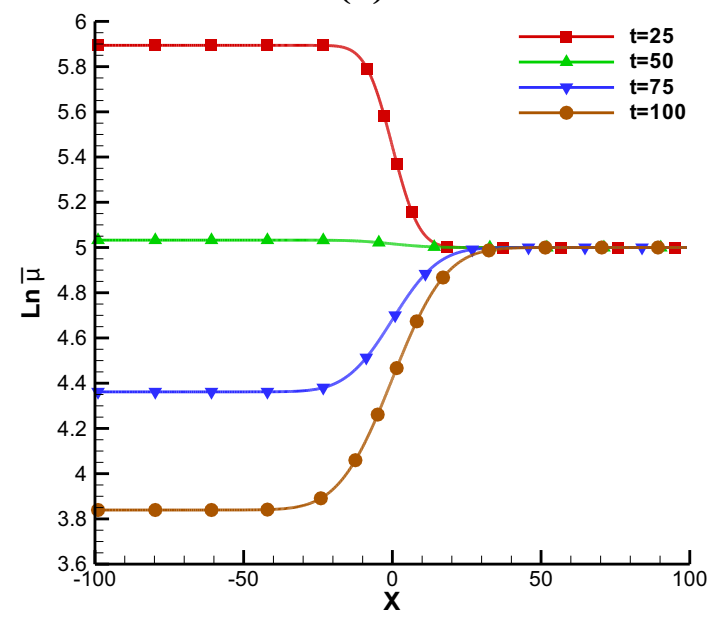

Figure 3.12: (a) variation of the base state concentration along the channel, (b) variation of the base state viscosity along the channel, $R_{n}=5, \delta_{b}=1, \delta_{n}=1, D a_{d e p}=0.01$

effect is well illustrated in Fig. 3.13 depicting results for $\delta_{b}=\delta_{n}=1, D a_{d e p}=0.01$ and two values; $R_{n}=1$ and $R_{n}=3$. It is clear that an increase of $R_{n}$ tends to attenuate the instability. However, as NPs are removing from the system their effect is less pronounced. The analysis of the breakthrough time and ML for different nanofluid viscosity ratios shown in Fig. 3.14 conforms the qualitative results. These trends towards attenuating the instability also confirm the prediction from LSA in the previous section where it was found that a larger $R_{n}$ systematically results in smaller growth rates and a reduction of the spectrum of unstable wave numbers (Fig. 3.3).

Diffusion can play an important role in controlling the initial instabilities in the flow. It is known that the instabilities will grow when the viscosity is monotonically increasing along the channel. However diffusion rate of each component can have a significant effect on the redistribution of viscosity in the system. Depending on other properties, diffusion can have either stabilizing and destabilizing effects in an unstable flow or make a stable system unstable [4]. At this stage, the aim is to investigate the effect of both NPs and fluids diffusion rate when the flow is initially unstable $\left(R_{b}>R_{a}\right)$ and in case of $R_{n}>0$. Fig. 3.15 shows that as the diffusion of NPs increases the flow is more unstable. This effect is more obvious in higher nanofluid viscosity ratios and specially in the early stages of the grow where the 

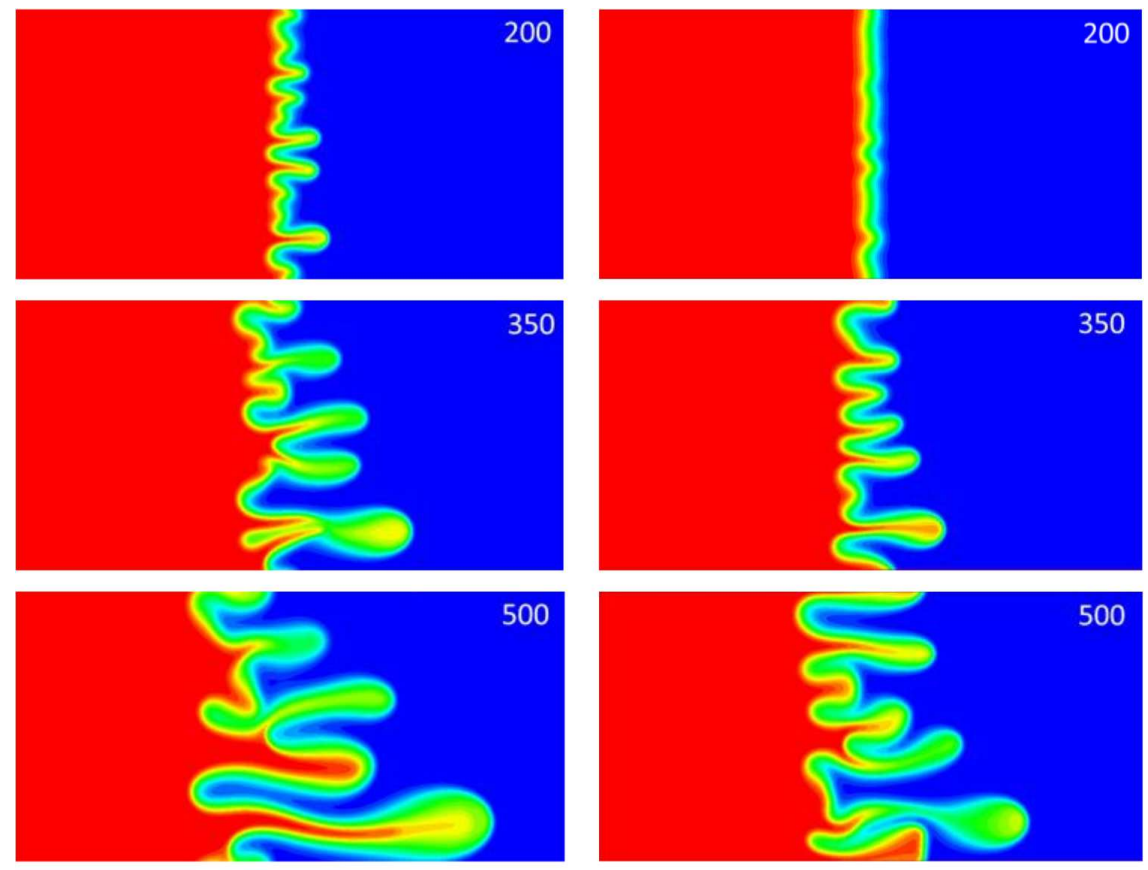

(a) $R_{n}=1$

(b) $R_{n}=3$

Figure 3.13: Contours of $C_{a}$ for different nanofluid viscosity ratio $\delta_{b}=1, \delta_{n}=1, D a_{\text {dep }}=0.01$

(a)

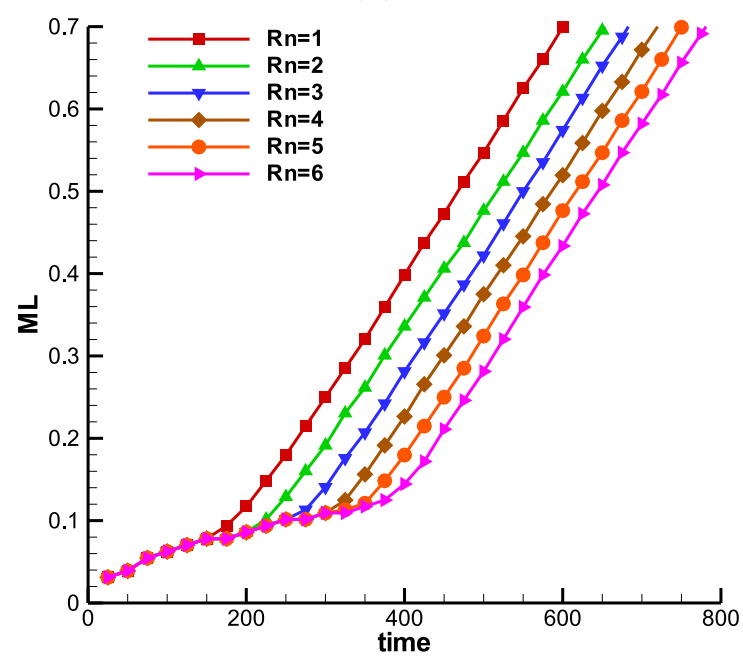

(b)

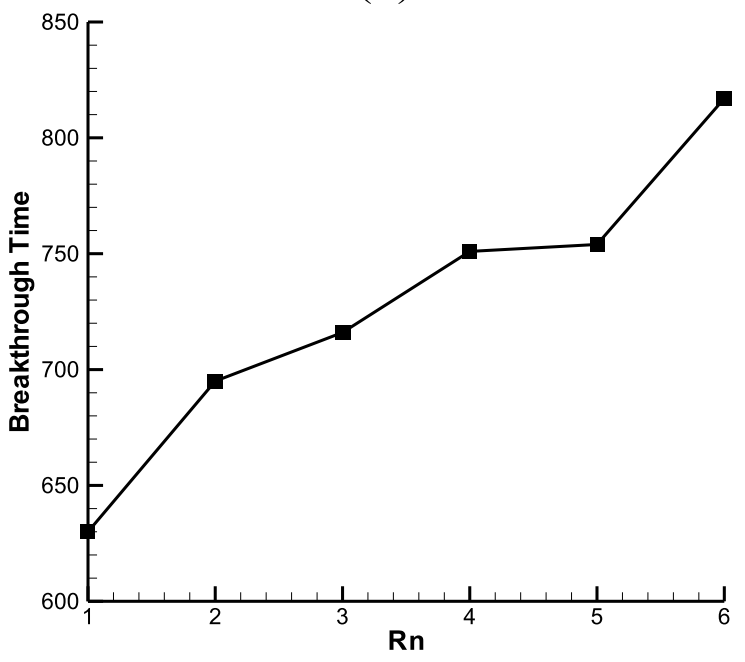

Figure 3.14: (a) Mixing length, (b) breakthrough time, at different nanofluid viscosity ratios, $\delta_{b}=1, \delta_{n}=1, D a_{\text {dep }}=0.01$ 

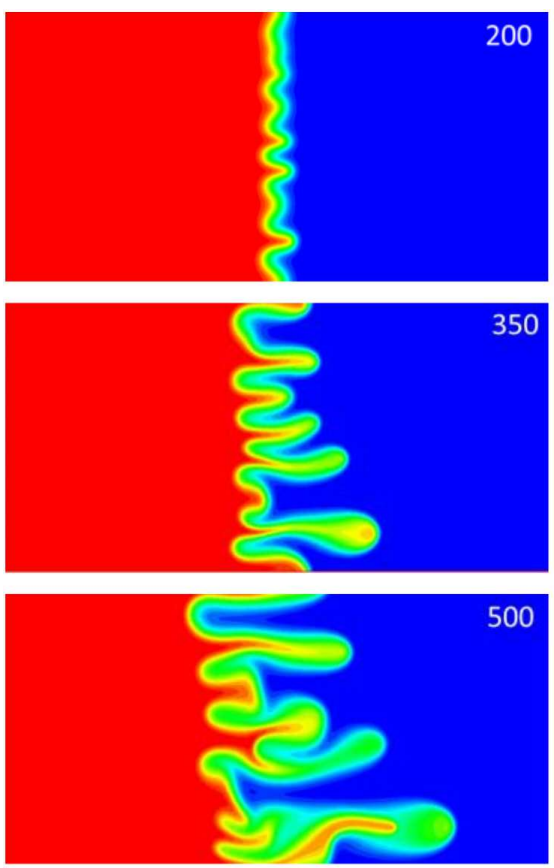

(a) $\delta_{n}=0.1$
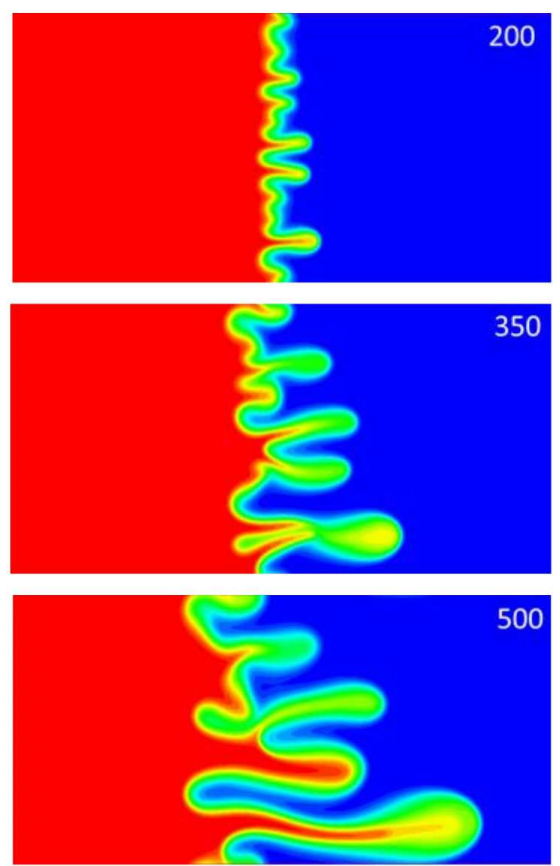

(b) $\delta_{n}=1$

Figure 3.15: Contours of $C_{a}$ for different NP diffusion rate $R_{n}=1, \delta_{b}=1, D a_{\text {dep }}=0.01$

NPs are exist in the system with higher concentrations. This result confirms the prediction of LSA where it was found that larger values of $\delta_{n}$ lead to a more unstable displacement (see Fig. 3.5). Further quantitative analysis depicted in Fig. 3.16 confirms that higher values of NP diffusion lead to a more unstable situation with higher ML. Moreover, the variation of the transversely averaged concentration along the channel indicates that at higher values of $\delta_{n}$, the instabilities cause fluid A to penetrate further downstream in comparison with lower $\delta_{n}$.

The effect of $\delta_{b}$ is shown in Fig. 3.17. It is clear that $\delta_{b}$ has a strong effect such that even a small deviation from $\delta_{b}=1$ to the lower or upper values changes the flow pattern significantly (compare with Fig. 3.13a). As shown the number of fingers in the early stages of the flow increases as $\delta_{b}$ is decreased and that flows involving a displaced fluid with a smaller diffusion coefficient than that of the displacing one, lead to a clearly more unstable displacement and more complex finger structures. These results are in concordance with the predictions of the LSA where it was found that a smaller $\delta_{b}$ leads to a wider spectrum of unstable modes and 
(a)

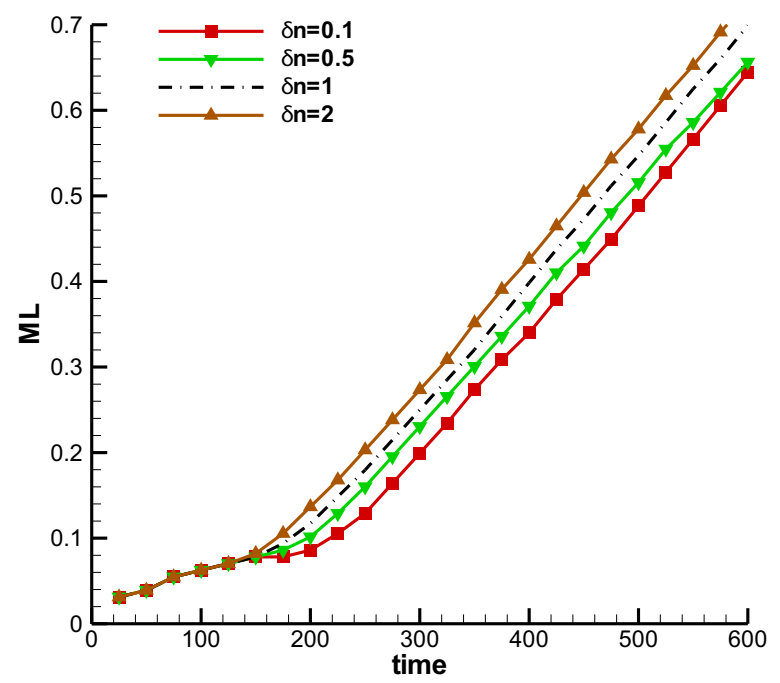

(b)

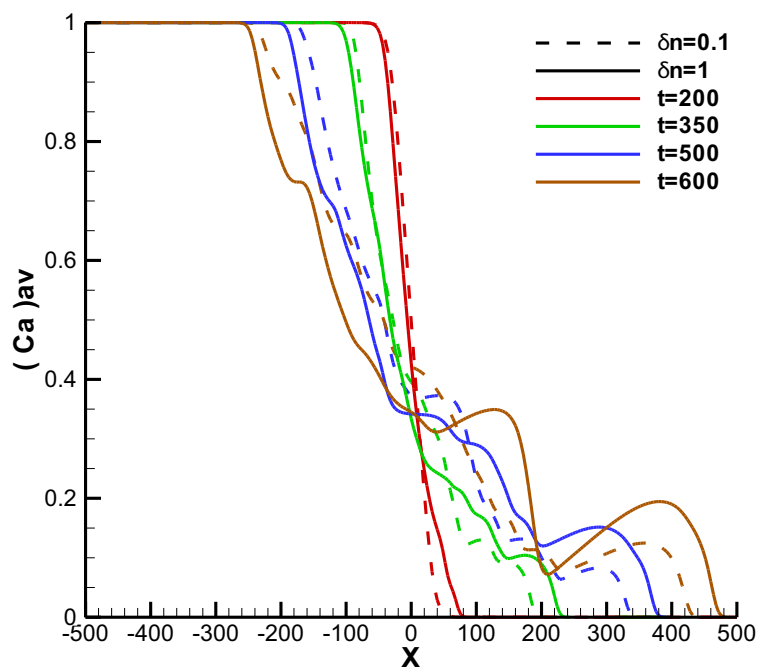

Figure 3.16: (a) Variation of mixing length with time (b) transversely averaged concentration along the channel, $R_{n}=1, \delta_{b}=1, D a_{d e p}=0.01$

larger growth rates. Furthermore, the tip splitting configuration of the fingers represents the higher rate of cross flow, more stretched front and steeper concentration gradient near the front which is a suitable condition for small disturbances to grow. The higher rate of cross flow causes the initial small disturbances at the front to diffuse and grow faster and make the flow more unstructured. Hence, as time passes, finger splits stretch more and become narrower and if $\delta_{b}$ is small enough, interact with each other and fade away. This constitutes a good condition for enhancing fluid mixing in very low $R e$ numbers flows. However for $\delta_{b}>1$, the sharp viscosity gradient fades away and the disturbances cannot grow easily.

In order to dissociate the role of NPs from that of the rates of diffusion of the species, Fig. 3.18 depicts results for $\delta_{b}=1.5$ in the presence and absence of NPs. The latter case corresponds to the study of Mishra et al.[4]. It is clear that although the presence of NPs makes the system less unstable, it does not however induce major changes in the finger overall structures. 

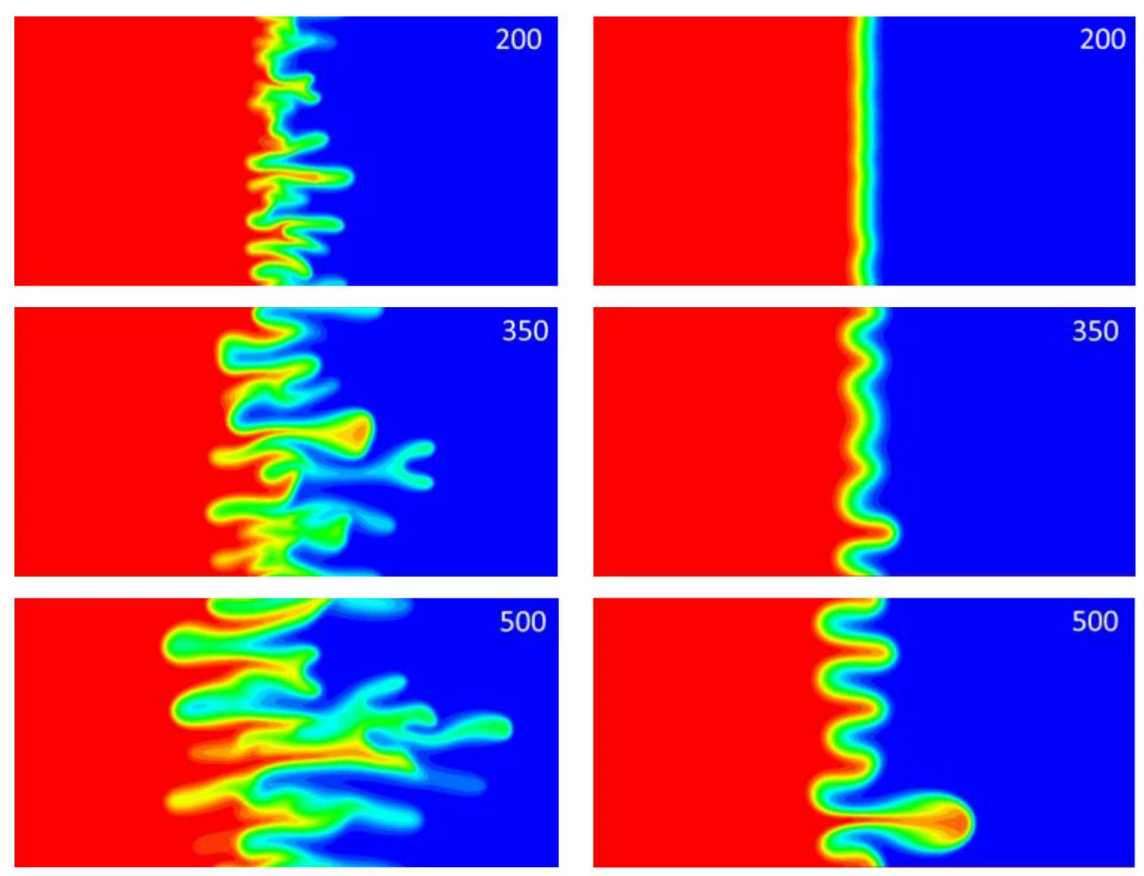

(a) $\delta_{b}=0.6$

(b) $\boldsymbol{\delta}_{\boldsymbol{b}}=\mathbf{1 . 5}$

Figure 3.17: Contours of $C_{a}$ for different diffusion rate of displaced fluid $R_{n}=1, \delta_{n}=1$, $D a_{d e p}=0.01$

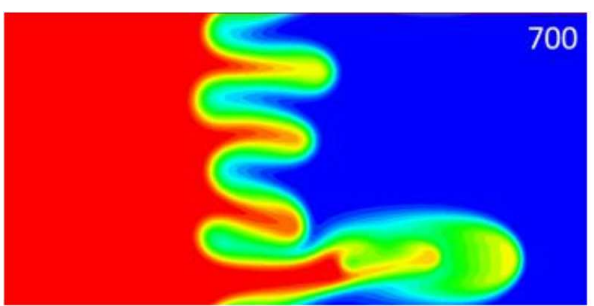

(a) $R_{n}=\mathbf{0}$

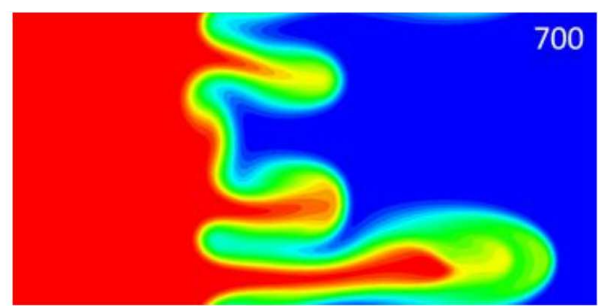

(b) $R_{n}=1$

Figure 3.18: Finger configuration when $\delta_{b}=1.5$ in both (a) particle-free and (b) particleincluded $\delta_{n}=1, D a_{\text {dep }}=0.01$ systems 


\section{Chapter 4}

\section{Thermophoretic Effects on}

\section{Instabilities of Nanoflows in Porous}

\section{Media}

1 The objective of this study is to investigate the role of parameters affecting the development and growth of viscous fingering (VF) instabilities of non-isothermal nanoflows in homogeneous porous media. More specifically, the study will analyze the combined effects of Brownian diffusion and thermophoresis, the average motion of nanoparticles (NPs) resulting from the temperature gradient, on the flow dynamics, particle distribution and the instability. The use of thermophoretic effects and the potential for the transport of particles by temperature gradients has been applied in a number of processes that include thermal fieldflow fractionation where micro and nano-scale particles can be separated from the solvent by imposing temperature gradient [79], ultrafine particle collection [80], and the design of thermophoretic swimmers where particles are self-propelled as a result of anisotropic heating of the surrounding fluid [81]. Accordingly, thermophoresis as an additional transport

\footnotetext{
${ }^{1}$ This chapter is based on the following journal article:

B. Dastvareh, J. Azaiez, "Thermophoretic Effects on Instabilities of Nanoflows in Porous Media", Journal of Fluid Mechanics, vol. 857, (2018) 173-199.
} 
mechanism of NPs that may affect the NP laden VF instability observed in applications such as nano-drug delivery [16], particles mixing in microfluidic devises [17] and the heavy oil upgrading and recovery in the presence of nano-catalysts [82].

In this study, first the effects of Brownian diffusion and thermophoresis are analyzed in two representative unstable systems; HDC (Hot fluid Displaces Cold fluid) and CDH (Cold fluid Displaces Hot fluid). The analysis is conducted using the concentration distribution of the displaced fluid, resulting from direct non-linear simulations of the flow. The observed phenomena are explained in terms of the viscosity and NP concentration distributions and through quantitative analyses. Physical interpretations of the observed trends are presented. Then the study is extended to examine the effects of thermophoresis in the case of initially stable systems.

\subsection{Physical problem}

A two dimensional homogeneous porous medium with constant intrinsic permeability $K$ or equivalently a horizontal Hele-Shaw cell is considered, where fluid B with initial concentration $C_{b 0}$, viscosity $\mu_{b 0}=\mu\left(0, C_{b 0}, 0\right)$ and temperature $T_{b}$ is at rest. A miscible fluid $\mathrm{A}$ with initial concentration $C_{a 0}$, viscosity $\mu_{a 0}=\mu\left(C_{a 0}, 0,0\right)$ and temperature $T_{a}$ containing NPs with initial concentration $C_{n 0}$, is injected at a constant velocity $U$ to displace fluid $\mathrm{B}$. The viscosity of the resulting initial nanofluid (fluid A containing NPs) is $\mu_{n 0}=\mu\left(C_{a 0}, 0, C_{n 0}\right)$ and the NPs are assumed to be in thermal equilibrium with the host fluid. Furthermore, the fluids are assumed to be incompressible and the nanofluid is dilute. The length and width of the medium are $L$ and $W$ as shown in Fig. 4.1. 


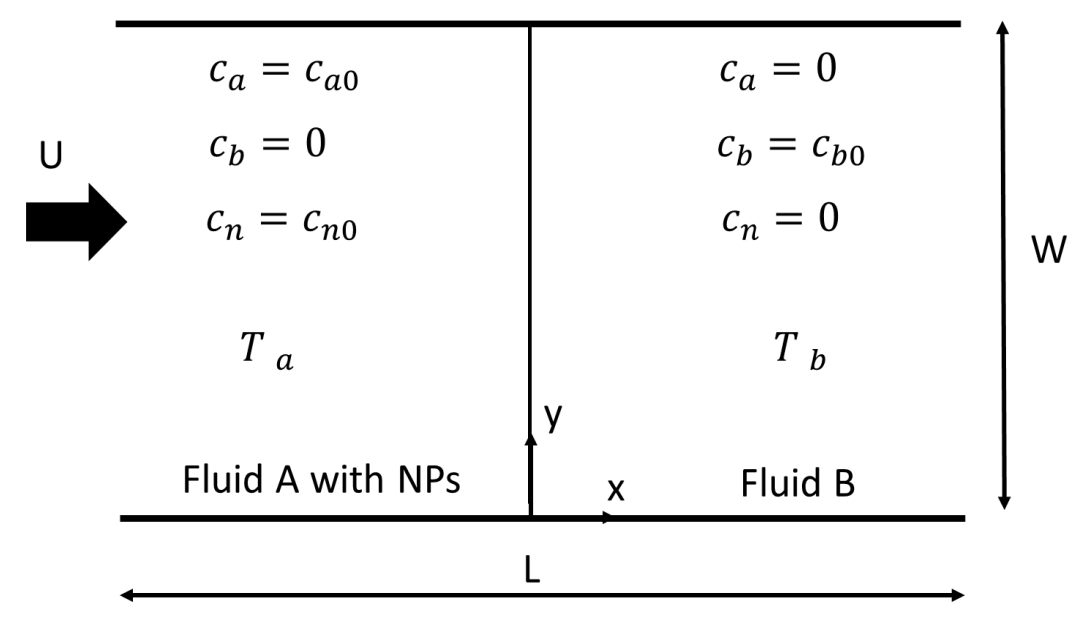

Figure 4.1: Schematic view of the flow geometry.

\subsection{Problem formulation}

The flow is modeled with the following equations:

$$
\begin{gathered}
\vec{\nabla} \cdot \vec{V}_{D}=0 \\
\vec{\nabla} P=-\frac{\mu}{K} \vec{V}_{D} \\
\frac{\partial C_{b}}{\partial t}+\frac{1}{\phi}\left(\overrightarrow{V_{D}} \cdot \vec{\nabla} C_{b}\right)=D_{b a} \nabla^{2} C_{b} \\
\frac{\partial C_{n}}{\partial t}+\frac{1}{\phi}\left(\overrightarrow{V_{D}} \cdot \vec{\nabla} C_{n}\right)=\vec{\nabla} \cdot\left(D_{n} \vec{\nabla} C_{n}+D_{T} \frac{\vec{\nabla} T}{T}\right)-k_{d e p} C_{n} \\
\frac{\partial T}{\partial t}+\frac{\lambda}{\phi}\left(\vec{V}_{D} \cdot \vec{\nabla} T\right)=\vec{\nabla} \cdot(\alpha \vec{\nabla} T)+\beta\left(D_{n} \vec{\nabla} C_{n} \cdot \vec{\nabla} T+D_{T} \frac{\vec{\nabla} T \cdot \vec{\nabla} T}{T}\right)
\end{gathered}
$$

The continuity equation and Darcy's law (Eqs. 4.1, 4.2) are used for the conservation of mass and momentum, where $\vec{V}_{D}(u, v)$ is Darcy's velocity, $P$ the local pressure, $\mu$ the viscosity and $K$ the intrinsic permeability. The flow is also governed by the advection diffusion equations for the transport of fluids and NPs. One expects that in multi-component systems like the one considered here, the flux of one component is affected not only by its own concentration gradient, but also by other driving forces including the concentration gradients of other components and the temperature gradient. However, since the mass fraction of the NPs is 
very low, the mass averaged velocity of the system in the absence of bulk motion is essentially the same as that of the solvent (fluid) velocity [83]. This implies that the diffusion of the NPs does not affect the molecular flux of the miscible base fluids. Accordingly, it is legitimate to neglect the NP flux effect on the transport of the fluids (Eq. 4.3). Furthermore, the fluids molecular flux effect on the transport of the NPs and the temperature gradient effect on the transport of the fluids (Soret effect) are ignored.

It is well-known that NPs increase the viscosity, thermal conductivity and the convective heat transfer coefficient of the base fluid [56-58]. Buongiorno [84] attributed the abnormal increase of the convective heat transfer coefficient to the NP/base fluid relative (slip) velocity. As a result of his analysis of the effect of seven different slip mechanisms, it was concluded that Brownian diffusion and thermophoresis (particle migrations under the effect of temperature gradient) are the most important slip mechanisms. Accordingly, he proposed a two component (NP-fluid) model for the mass and heat transport in nanofluids. Eq. 4.4 is based on his model for the transport of NPs and is adapted for flows in porous media [85]. The first and second terms in the RHS of this equation represent the net rate of change of NP concentration as a result of Brownian diffusion and thermophoresis, respectively. In equations 4.3 and $4.4, \phi$ is the medium porosity, $C_{i}$ the mass fraction or volume fraction (in ideal solution), $T$ the absolute temperature, $D_{b a}$ the mutual diffusion coefficient of the fluids and $D_{n}$ and $D_{T}$ are the diffusion coefficient of NPs resulting from Brownian motion and thermophoresis. Finally, the energy equation (Eq. 4.5) is also used to model this nonisothermal system, where the last two terms represent the contributions of the Brownian motion and thermophoresis. In the energy equation, $\lambda$ is the thermal lag coefficient, $\alpha$ the thermal diffusivity and $\beta$ the relative volumetric heat capacity of the NPs to that of the medium.

$$
\lambda=\frac{\phi\left(\rho c_{p}\right)_{n f}}{\left(\rho c_{p}\right)_{m}}, \quad \alpha=\frac{k_{m}}{\left(\rho c_{p}\right)_{m}}, \quad \beta=\frac{\phi\left(\rho c_{p}\right)_{p}}{\left(\rho c_{p}\right)_{m}}
$$


Where:

$$
\begin{gathered}
\left(\rho c_{p}\right)_{m}=\phi\left(\rho c_{p}\right)_{n f}+(1-\phi)\left(\rho c_{p}\right)_{s} \\
k_{m}=\phi k_{n f}+(1-\phi) k_{s}
\end{gathered}
$$

In the above equations, $k$ is the thermal conductivity and $\rho c_{p}$ the volumetric heat capacity. The subscripts $m, p, s$ and $n f$ stand for the medium, NP, solid and nanofluid respectively. In the present model $\alpha$ is assumed to be constant since the thermal conductivity of the porous medium is mostly dominated by the solid phase, rather than the NPs. Even in the case of a Hele-Shaw cell, it is legitimate to take a constant value for the thermal conductivity of nanofluid $\left(k_{n f}\right)$ as one of its properties. $\left(\rho c_{p}\right)_{n f}$ is also assumed constant by a similar argument. In Eq. 4.4, $k_{\text {dep }}$ is the deposition rate described with the widely applied colloidal filtration theory (CFT) $[67,86]$. A list of deposition models along with their pros and cons according to experimental data can be found in $[87,88]$.

To complete the formulation, a brief discussion of thermophoresis is presented. Thermophoresis is an additional transport mechanism of particles dispersed in a continuum aside from Brownian diffusion. It is associated with temperature gradients and is the equivalent of the Soret effect in gaseous and liquid mixtures. Physically, it represents the time-averaged chaotic impulse of the surrounding fluid on the particles [89]. It must be noted that due to this random force, particles move randomly in all directions, however their average displacement is slightly biased to the opposite direction of the temperature gradient. From Eq. 4.4, the total mass flux of particles aside from convection, is:

$$
\frac{\overrightarrow{J_{p}}}{\rho_{p}}=-D_{n} \vec{\nabla} C_{n}-D_{T} \frac{\vec{\nabla} T}{T}
$$

The Brownian diffusion coefficient can be expressed with the Stokes-Einstein equation, $D_{n}=\frac{k_{B} T}{3 \pi \mu_{b f} d_{p}}$, where $k_{B}$ is the Boltzmann constant, $\mu_{b f}$ viscosity of the base fluid and $d_{p}$ the diameter of the particles. On the other hand, although different correlations for 
thermophoretic velocity and accordingly thermophoretic diffusivity have been introduced in gaseous mixtures, there are rather limited studies when it comes to liquids. A thermophoretic diffusion coefficient based on experimental data reported by [90] for relatively large $\left(d_{p} \sim 1 \mu m\right)$ latex spheres particles in water and n-hexane is expressed as follows [84]:

$$
D_{T}=-K_{t h} \frac{\mu_{b f}}{\rho_{b f}} C_{n}
$$

Where $\rho_{b f}$ is the density of the base fluid and $K_{t h}=0.26 \frac{k_{b f}}{k_{b f}+k_{p}}$. Since Eq. 4.9 is derived for very large particles, it would result in very small thermophoretic diffusion coefficient for NPs. Furthermore, it suggests almost zero $D_{T}$ for highly conductive NPs $\left(k_{p}>>k_{b f}\right)$ which does not concord with experimental measurements involving nanoflows [91, 92] and indicates that this model is not fit to represent the thermophoretic diffusivity in nanofluids. Recently, Michaelides [89] proposed a model for the thermophoretic velocity of NPs in liquids, and suggested that it depends strongly on the size of the NPs. The model which is validated with experimental data suggests fairly considerable values of the thermophoretic velocity and accordingly thermophoretic diffusivity of NPs. The thermophoretic diffusion coefficient derived from his model is similar to Eq. 4.9 with $K_{t h}=A_{0}\left(\frac{r_{p}}{r_{p_{0}}}\right)^{-B_{0}}$ where $r_{p}$ is the radius of the NPs in nanometer, $r_{p_{0}}=1 \mathrm{~nm}$, while $A_{0}$ and $B_{0}$ are experimental constants that depend on the NP/base fluid system [89].

Thermophoretic diffusion of particles is typically expressed in the literature by the thermophoretic mobility $\hat{D_{T}}=\frac{D_{T}}{C_{n} T}\left[\frac{m^{2}}{s K}\right]$ or by $\widetilde{D_{T}}=\frac{D_{T}}{C_{n}}\left[\frac{m^{2}}{s}\right]$ known as the true thermophoretic diffusion coefficient [93]. Although very diverse values can be found in the literature, in most investigated systems the thermophoretic mobility varies within the fairly limited range of $10^{-12}<{\hat{D_{T}}}_{T}<10^{-11} \frac{\mathrm{m}^{2}}{\mathrm{sK}}$ [93]. Accordingly, the value of the true thermophoretic diffusion coefficient is in the order of $\widetilde{D_{T}}<O\left(10^{-9}\right) \frac{m^{2}}{s}$. However larger values; $O\left(10^{-10}\right) \frac{m^{2}}{s K}$ for $\hat{D_{T}}$ or $O\left(10^{-8}\right) \frac{m^{2}}{s}$ for $\widetilde{D_{T}}$ have also been reported in some studies dealing with metal NPs [92]. Values of the true thermophoretic diffusion coefficient $\widetilde{D_{T}}$ for some metal or metal-oxide NPs 
derived from the correlation introduced by [89] can even reach to $O\left(10^{-7}\right) \frac{m^{2}}{s}$.

Regarding the relative importance of Brownian vs thermophoretic diffusions, small values of $\delta=\frac{\widetilde{D_{T}}}{D_{n}}$; as low as zero (or $\delta<0$, which is not the focus of this study) as well as large ones; e.g. $\delta=156(R=53 \mathrm{~nm})$ and $\delta=641(R=253 \mathrm{~nm})$ for polystyrene (PS) spheres in aqueous systems have been reported [94]. Accordingly, the effects of thermophoresis can be very important compared to those of Brownian diffusion and therefore cannot be ignored when modeling the transport of NPs.

In this study the Brownian and thermophoretic diffusion coefficients are assumed to be linear functions of $T$ and $C_{n}$ respectively, and to depend on the base fluid constant viscosity and density [84]. Accordingly, one may express them as $D_{n}=D_{n_{0}} \frac{T}{T_{a}}$ and $D_{T}=D_{T_{0}} \frac{C_{n}}{C_{n 0}}$ where $D_{n_{0}}$ and $D_{T 0}$ are constants. It must be noted that $D_{T}$ must not be treated as constant, as this may lead to unphysical negative values of the NP concentrations, especially when the NP concentration gradient and temperature gradient are in opposite directions. This is a common mis-application encountered in the literature involving numerical simulations of nanoflows based on Buongiorno's model, e.g. [95].

The governing equations are made dimensionless and then formulated in a reference frame moving at the injection velocity $U$. Note that since the permeability $K$ is constant, it is incorporated in the definition of the viscosity such that, henceforth $\frac{\mu}{K}$ stands for $\mu$.

$$
\begin{gathered}
x^{*} / y^{*}=\frac{x / y}{\frac{D_{b a} \phi}{U}}, \vec{V}^{*}=\frac{\vec{V}_{D}}{U}, t^{*}=\frac{t}{\frac{D_{b a} \phi^{2}}{U^{2}}}, P^{*}=\frac{P}{\mu_{a 0} D_{b a} \phi} \\
\mu^{*}=\frac{\mu}{\mu_{a 0}}, C_{b}^{*}=\frac{C_{b}}{C_{b_{0}}}, C_{n}^{*}=\frac{C_{n}}{C_{n_{0}}}, \theta=\frac{T}{T_{a}}
\end{gathered}
$$


The dimensionless equations where the asterisks have been dropped for convenience, are:

$$
\begin{gathered}
\vec{\nabla} \cdot \vec{V}=0 \\
\vec{\nabla} P=-\mu(\vec{V}+\vec{i}) \\
\frac{\partial C_{b}}{\partial t}+\vec{V} \cdot \vec{\nabla} C_{b}=\nabla^{2} C_{b} \\
\frac{\partial C_{n}}{\partial t}+\vec{V} \cdot \vec{\nabla} C_{n}=\delta_{n} \theta \nabla^{2} C_{n}+\left(\delta_{n}+\frac{\delta_{T}}{\theta}\right) \vec{\nabla} \theta \cdot \vec{\nabla} C_{n}+\left(\delta_{T} \vec{\nabla} \cdot\left(\frac{\vec{\nabla} \theta}{\theta}\right)-D a_{d e p}\right) C_{n} \\
\frac{\partial \theta}{\partial t}+(\lambda-1) \frac{\partial \theta}{\partial x}+\lambda(\vec{V} \cdot \vec{\nabla} \theta)=L e \nabla^{2} \theta+\epsilon\left(\delta_{n} \theta \vec{\nabla} C_{n} \cdot \vec{\nabla} \theta+\delta_{T} C_{n} \frac{\vec{\nabla} \theta \cdot \vec{\nabla} \theta}{\theta}\right)
\end{gathered}
$$

Where:

$$
\begin{gathered}
\delta_{n}=\frac{D_{n_{0}}}{D_{b a}}, \quad \delta_{T}=\frac{D_{T_{0}}}{D_{b a} C_{n_{0}}}, \quad L e=\frac{\alpha}{D_{b a}} \\
D a_{d e p}=\frac{k_{d e p} D_{b a} \phi^{2}}{U^{2}}, \quad \epsilon=\frac{C_{n_{0}} \phi\left(\rho c_{p}\right)_{p}}{\left(\rho c_{p}\right)_{m}}
\end{gathered}
$$

In the above equations $L e$ is Lewis number, $D a_{d e p}$ the deposition rate coefficient while $\delta_{n}$ and $\delta_{T}$ are referred to as the Brownian and the thermophoretic diffusivity, respectively. It should be noted that the present study is limited to displacements with no NP deposition, and henceforth $D a_{d e p}=0$. This is a reasonable assumption as NP deposition in porous media can indeed be avoided through the use of either surfactants or surface charge technology [96].

Dimensionless geometric quantities result from the domain boundaries which are $\left(-\frac{P e}{2}, \frac{P e}{2}\right)$ in the $\mathrm{x}$-direction and $\left(0, \frac{P e}{A_{s}}\right)$ in the $\mathrm{y}$-direction, where $A_{s}=L / W$ is the domain aspect ratio and $P e=\frac{U L}{\phi D_{b a}}$ is the Péclet number. The boundary conditions in this reference frame consist of zero flux for concentrations and temperature in the x-direction, and periodicity in the y-direction:

$$
\begin{gathered}
(u, v)\left(-\frac{P e}{2}, y, t\right)=(u, v)\left(\frac{P e}{2}, y, t\right)=(0,0) \\
\left(\frac{\partial C_{b}}{\partial x}, \frac{\partial C_{n}}{\partial x}, \frac{\partial \theta}{\partial x}\right)\left(-\frac{P e}{2}, y, t\right)=\left(\frac{\partial C_{b}}{\partial x}, \frac{\partial C_{n}}{\partial x}, \frac{\partial \theta}{\partial x}\right)\left(\frac{P e}{2}, y, t\right)=(0,0,0) \\
\left(u, v, C_{b}, C_{n}, \theta\right)(x, 0, t)=\left(u, v, C_{b}, C_{n}, \theta\right)\left(x, \frac{P e}{A_{s}}, t\right)
\end{gathered}
$$


One must note that due to thermophoresis, zero flux NP concentration in the x-direction,

requires that $\delta_{n} \theta \frac{\partial C_{n}}{\partial x}+\frac{\delta_{T} C_{n}}{\theta} \frac{\partial \theta}{\partial x}=0$, which is automatically satisfied by the conditions $\frac{\partial C_{n}}{\partial x}=$ $\frac{\partial \theta}{\partial x}=0$.

To complete the model a form for the dependence of the viscosity on concentration and temperature must be specified. Following previous studies, an exponential viscosityconcentration-temperature relationship is adopted [22, 42]:

$$
\mu=\exp \left(R_{b} C_{b}+R_{\theta} \frac{1-\theta}{1-r}+R_{n} C_{n}\right)
$$

Where $r=T_{b} / T_{a}$ is the temperature ratio of the displaced fluid to the displacing one, while $R_{b}, R_{\theta}$ and $R_{n}$ are mobility ratios defined as:

$$
R_{b}=\ln \left(\frac{\mu_{b_{0}}}{\mu_{a_{0}}}\right)_{T_{a}}, \quad R_{\theta}=\ln \left(\frac{\mu_{T_{b}}}{\mu_{T_{a}}}\right), \quad R_{n}=\ln \left(\frac{\mu_{n_{0}}}{\mu_{a_{0}}}\right)_{T_{a}}
$$

In particular, $R_{b}>0\left(R_{b}<0\right), R_{\theta}=R_{n}=0$ represents an unstable (stable) isothermal NP-free system where a low (high) viscosity fluid displaces a high (low) viscosity one, while $R_{\theta}>0\left(R_{\theta}<0\right), R_{b}=R_{n}=0$ represents an unstable (stable) non-isothermal NP-free system where a hot (cold) fluid displaces a cold (hot) fluid. Flows with $R_{n}>0$ represent the case where addition of NPs increases the viscosity of the displacing base fluid. Note that $R_{\theta}$ and $r$ enter the model as a group; $R_{\theta}^{\prime}=\frac{R_{\theta}}{1-r}$.

\subsection{Numerical method}

Following the procedure expressed in section 3.3, the governing equations are first formulated in terms of the vorticity $(\omega)$, stream-function $(\psi)$, concentrations $\left(C_{i}\right)$ and temperature $(\theta)$. Then the resulting equations are solved numerically using the Hartley based Pseudo-Spectral 
method [77]. The base state concentrations and temperature are derived from:

$$
\begin{gathered}
\frac{\partial \bar{C}_{b}}{\partial t}=\frac{\partial^{2} \bar{C}_{b}}{\partial x^{2}} \\
\frac{\partial \bar{C}_{n}}{\partial t}=\delta_{n} \bar{\theta} \frac{\partial^{2} \bar{C}_{n}}{\partial x^{2}}+\left(\delta_{n}+\frac{\delta_{T}}{\bar{\theta}}\right) \frac{\partial \bar{\theta}}{\partial x} \frac{\partial \bar{C}_{n}}{\partial x}+\left(\delta_{T} \frac{\partial}{\partial x}\left(\frac{1}{\bar{\theta}} \frac{\partial \bar{\theta}}{\partial x}\right)-D a_{d e p}\right) \bar{C}_{n} \\
\frac{\partial \bar{\theta}}{\partial t}+(\lambda-1) \frac{\partial \bar{\theta}}{\partial x}=L e \frac{\partial^{2} \bar{\theta}}{\partial x^{2}}+\epsilon\left(\delta_{n} \bar{\theta} \frac{\partial \bar{C}_{n}}{\partial x} \frac{\partial \bar{\theta}}{\partial x}+\frac{\delta_{T} \bar{C}_{n}}{\bar{\theta}}\left(\frac{\partial \bar{\theta}}{\partial x}\right)^{2}\right)
\end{gathered}
$$

It should to be mentioned that the base state velocities are zero in the moving reference frame. Zero flux boundary conditions are used to solve the above-mentioned base state equations. The solution of Eq. 4.20 is determined analytically as:

$$
\bar{C}_{b}(x, t)=\frac{1}{2} \operatorname{erfc}\left(\frac{-x}{2 \sqrt{t}}\right)
$$

Furthermore, the coupled Eq. 4.21 and 4.22 are solved numerically with the method of lines. The equations for the perturbation terms are:

$$
\begin{gathered}
\nabla^{2} \psi=-\omega \\
\omega=R_{b} N_{b}-\frac{R_{\theta}}{1-r} N_{\theta}+R_{n} N_{n} \\
\frac{\partial C_{b}{ }^{\prime}}{\partial t}=J_{b}+\frac{\partial^{2} C_{b}{ }^{\prime}}{\partial x^{2}}+\frac{\partial^{2} C_{b}{ }^{\prime}}{\partial y^{2}} \\
\frac{\partial C_{n}{ }^{\prime}}{\partial t}=J_{n}+G_{n}-D a_{d e p} C_{n}{ }^{\prime} \\
\frac{\partial \theta^{\prime}}{\partial t}=\lambda J_{\theta}+(1-\lambda) \frac{\partial \theta^{\prime}}{\partial x}+\epsilon G_{\theta}+L e\left(\frac{\partial^{2} \theta^{\prime}}{\partial x^{2}}+\frac{\partial^{2} \theta^{\prime}}{\partial y^{2}}\right)
\end{gathered}
$$

Where:

$$
N_{i}=\frac{\partial \psi}{\partial x}\left(\frac{\partial \bar{X}_{i}}{\partial x}+\frac{\partial X_{i}^{\prime}}{\partial x}\right)+\left(1+\frac{\partial \psi}{\partial y}\right) \frac{\partial X_{i}^{\prime}}{\partial y}
$$




$$
\begin{gathered}
J_{i}=\frac{\partial \psi}{\partial x} \frac{\partial X_{i}^{\prime}}{\partial y}-\frac{\partial \psi}{\partial y}\left(\frac{\partial \bar{X}_{i}}{\partial x}+\frac{\partial X_{i}^{\prime}}{\partial x}\right) \\
G_{n}=\delta_{n}\left(\theta^{\prime} \frac{\partial^{2} \bar{C}_{n}}{\partial x^{2}}+\left(\bar{\theta}+\theta^{\prime}\right)\left(\frac{\partial^{2} C_{n}{ }^{\prime}}{\partial x^{2}}+\frac{\partial^{2} C_{n}{ }^{\prime}}{\partial y^{2}}\right)\right)-\frac{\delta_{T} \theta^{\prime}}{\bar{\theta}\left(\bar{\theta}+\theta^{\prime}\right)} \frac{\partial \bar{C}_{n}}{\partial x} \frac{\partial \bar{\theta}}{\partial x} \\
+\left(\delta_{n}+\frac{\delta_{T}}{\bar{\theta}+\theta^{\prime}}\right)\left(\frac{\partial \bar{C}_{n}}{\partial x} \frac{\partial \theta^{\prime}}{\partial x}+\frac{\partial C_{n}{ }^{\prime}}{\partial x} \frac{\partial \bar{\theta}}{\partial x}+\frac{\partial C_{n}{ }^{\prime}}{\partial x} \frac{\partial \theta^{\prime}}{\partial x}+\frac{\partial C_{n}{ }^{\prime}}{\partial y} \frac{\partial \theta^{\prime}}{\partial y}\right) \\
+\delta_{T}\left(\bar{C}_{n}+C_{n}{ }^{\prime}\right)\left[\frac{\partial}{\partial x}\left(\frac{\partial \theta^{\prime}}{\partial x}+\frac{\partial \bar{\theta}}{\partial x}\right)+\frac{\partial}{\partial y}\left(\frac{\partial \theta^{\prime}}{\bar{\theta} y}\right)\right]-\theta_{T} \bar{C}_{n} \frac{\partial}{\partial x}\left(\frac{\partial \bar{\theta}}{\bar{\theta}}\right) \\
G_{\theta}=\delta_{n} \theta^{\prime} \frac{\partial \bar{C}_{n}}{\partial x} \frac{\partial \bar{\theta}}{\partial x}+\frac{\delta_{T}}{\bar{\theta}+\theta^{\prime}}\left(\frac{\partial \bar{\theta}}{\partial x}\right)^{2}\left(C_{n}{ }^{\prime}-\frac{\overline{C_{n}} \theta^{\prime}}{\bar{\theta}}\right) \\
+\frac{\delta_{T}\left(\overline{C_{n}}+C_{n}{ }^{\prime}\right)}{\bar{\theta}+\theta^{\prime}}\left(\left(\frac{\partial \theta^{\prime}}{\partial x}\right)^{2}+\left(\frac{\partial \theta^{\prime}}{\partial y}\right)^{2}+2 \frac{\partial \bar{\theta}}{\partial x} \frac{\partial \theta^{\prime}}{\partial x}\right) \\
+\delta_{n}\left(\bar{\theta}+\theta^{\prime}\right)\left(\frac{\partial \bar{C}_{n}}{\partial x} \frac{\partial \theta^{\prime}}{\partial x}+\frac{\partial C_{n}{ }^{\prime}}{\partial x} \frac{\partial \bar{\theta}}{\partial x}+\frac{\partial C_{n}{ }^{\prime}}{\partial x} \frac{\partial \theta^{\prime}}{\partial x}+\frac{\partial C_{n}{ }^{\prime}}{\partial y} \frac{\partial \theta^{\prime}}{\partial y}\right)
\end{gathered}
$$

Where $X_{i}=\left(C_{i}, \theta\right)$. As discussed above $\omega(x, y, t), \psi(x, y, t), C_{i}{ }^{\prime}(x, y, t)$ and $\theta^{\prime}(x, y, t)$ are zero at the stream-wise boundaries and according to Eq. 4.17c they are periodic in the transverse direction. Moreover, zero values for vorticity and stream function, and a random number distribution for the concentration and the temperature perturbations are used as initial conditions.

In order to validate the code, the numerical convergence was checked, and it was found that a grid size of $256 \times 256$ is satisfactory. Furthermore, results were also validated by comparing with isothermal NP-laden and NP-free displacements. LSA was also carried out and the instability characteristic equation was derived for an arbitrary choice of parameters in a NP-laden non-isothermal system. The number of fingers predicted from the LSA was compared with that of the early growing fingers from the NLS. From the characteristic curve in Fig. 4.2, the critical wave number is $k_{c r} \approx 0.2$ resulting in a number of fingers $N F=\frac{k P e}{2 A_{s} \pi} \approx 16.3$. This is in concordance with the results of NLS at early times where $N F \approx 17$. This further confirms the validity of the nonlinear code. 


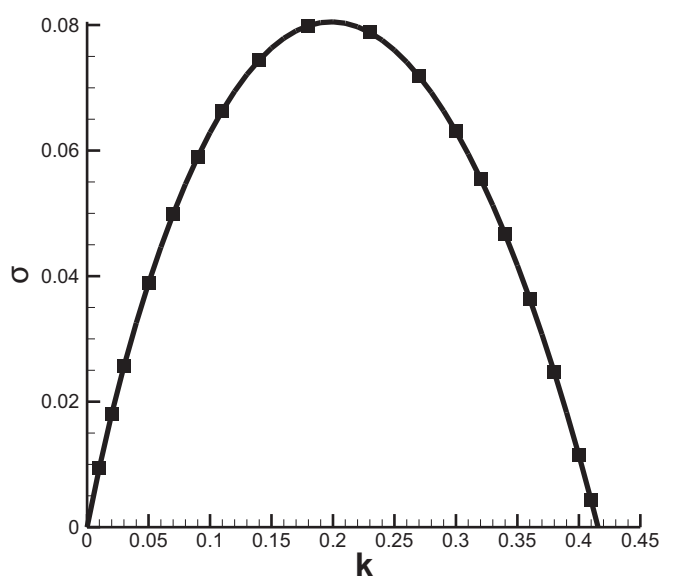

(a)

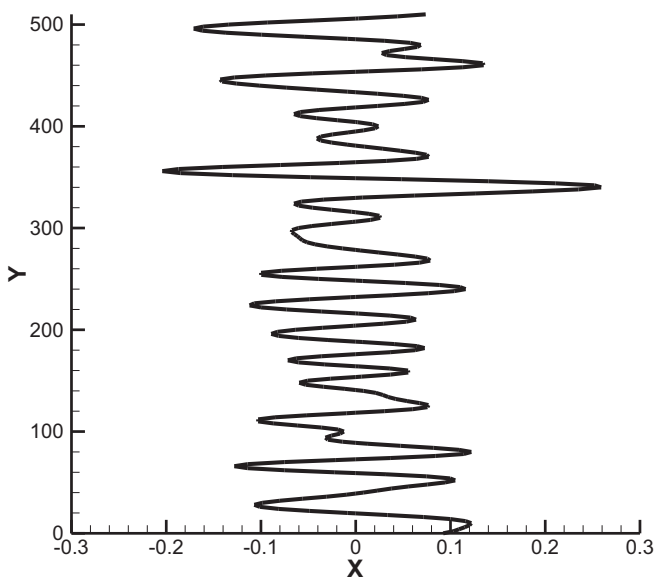

(b)

Figure 4.2: (a) Instability characteristics curve from LSA (b) iso-contour of $C_{b}=0.5$ at early times from NLS. $R_{b}=3, R_{\theta}=1, R_{n}=1, \lambda=0.8, \delta_{n}=1, \delta_{T}=3, L e=3, \epsilon=0.01$, $D a_{\text {dep }}=0, r=0.5, t_{0}=1, P e=1024, A_{s}=2$.

\subsection{Results and discusion}

Before presenting the results and discussion, a few adopted terminologies and reference parameters are defined. Throughout this study, an intrinsically unstable (stable) system corresponds to a system without NPs that is unstable (stable). This is to be contrasted with unstable (stable) systems that refer to unstable (stable) systems in the presence of NPs with or without heat transfer. Furthermore, this study focuses on NPs that increase the viscosity of the base fluid $\left(R_{n}>0\right)$.

Two types of non-isothermal displacements are considered; a Hot fluid Displacing a Cold one (HDC) where $R_{\theta}>0$ and $0<r<1$, and a Cold fluid Displacing a Hot one (CDH) where $R_{\theta}<0, r>1$. The reference parameters for the HDC system are: $R_{\theta}=1, r=0.5$ while they are $R_{\theta}=-1, r=2$ for the CDH system. Furthermore, unless mentioned otherwise, the other parameters are set as: $R_{b}=3, R_{n}=1, \lambda=1, \delta_{n}=1, \delta_{T}=3$, Le $=3, \epsilon=0.001, D a_{d e p}=$ 0, $P e=1024, A_{s}=2$ and $t_{0}=1$. The choice of these parameters represents unstable systems.

The qualitative characterization of the instability will be supported by a quantitative analysis based on transverse average concentrations $\left(C_{a v}\right)$ and the mixing length $(\mathrm{ML})$. The ML is defined as the ratio of the length within concentration range $C_{b, a v}=0.01$ to $C_{b, a v}=0.99$ 
to the length of the whole domain where:

$$
C_{b, a v}(x, t)=\frac{A_{s}}{P e} \int_{0}^{\frac{P e}{A_{s}}} C_{b}(x, y, t) d y
$$

Larger values of ML indicate a more unstable situation and vice versa.

In the previous Chapter it was found that the addition of NPs to the displacing fluid $\left(R_{n}>0\right)$ attenuates the instability of an intrinsically unstable solution as it decreases the viscosity contrast in the system. More specifically, it was found that in the absence of deposition and with the new set of parameters used here, the system is unstable if $R_{b}-R_{n}>0$ and is stable otherwise. However in the presence of deposition, NPs are gradually removed from the system and their effects on the viscosity of the fluids are weakened. As a result the instability is determined mainly by the viscosity ratio of the base fluids. In other words, if $R_{b}>0$ the system is unstable although the addition of NPs mitigates the instability. It implies that NPs $\left(R_{n}>0\right)$ cannot make an intrinsically unstable system stable in an isothermal case in the presence of deposition. It was also reported that Brownian diffusion increases the instability of an unstable system. However, it cannot make either stable or intrinsically stable systems, unstable. Unlike the isothermal flow, Brownian diffusion coefficient of the NPs $\left(D_{n}\right)$ here, varies with temperature. We will therefore start the discussion by analyzing its effects under non-isothermal conditions.

\subsubsection{Brownian diffusion}

Fig. 4.3 depicts concentration distributions of species $\mathrm{B}$ for the HDC and $\mathrm{CDH}$ case for two values of the Brownian diffusivity. Similar to the isothermal system, as $\delta_{n}$ increases both $\mathrm{HDC}$ and $\mathrm{CDH}$ systems exhibit more intricate finger configurations, reflecting the destabilizing effect of Brownian diffusion. Plots of the variation of the ML with time for different values of $\delta_{n}$ are presented for both systems in Fig. 4.4. The trends of a systematic increase of the ML with Brownian diffusion confirm the previous conclusion quantitatively. 


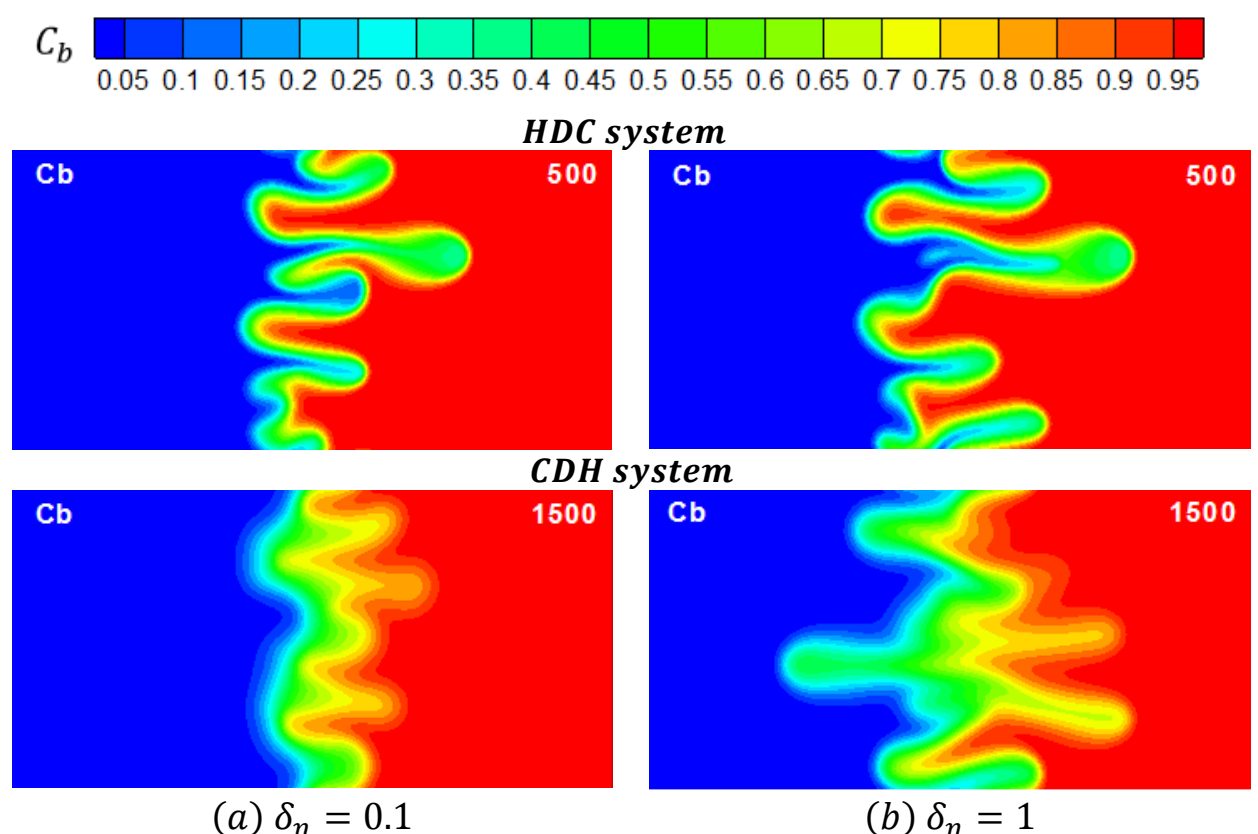

Figure 4.3: Contours of $C_{b}$ for two values of Brownian diffusivity in both HDC and CDH systems.

One may also note that the effects of $\delta_{n}$ are more prominent in the CDH system compared to the HDC one, and that the concentration distribution is more diffuse in the latter case. This points to an important role that the temperature probably plays in conjunction with Brownian diffusion in determining the distribution of NPs in the flow, and in turn in affecting the instability.

In order to analyze these effects, plots of the transversely averaged concentration of the NPs $\left(C_{n, a v}\right)$ are presented in Fig. 4.5 with an inset of NPs concentration contours at an instance in time. An examination of the concentration contours in the insets reveals that the distributions of the NPs are different in the HDC and CDH systems, and that in the latter one, changes in $\delta_{n}$ induce a dramatic variation of the distribution at the front. In particular, NPs in the HDC system move downstream easily in the low resistance paths provided by the growing fingers. On the other hand, in the CDH system, there is a resistance for the downstream transport of the NPs, which tend to accumulate at the front. For the small Brownian diffusivity, the NP concentration is characterized by a narrow band at the front with very large concentration values. This local accumulation of NPs leads to a local 


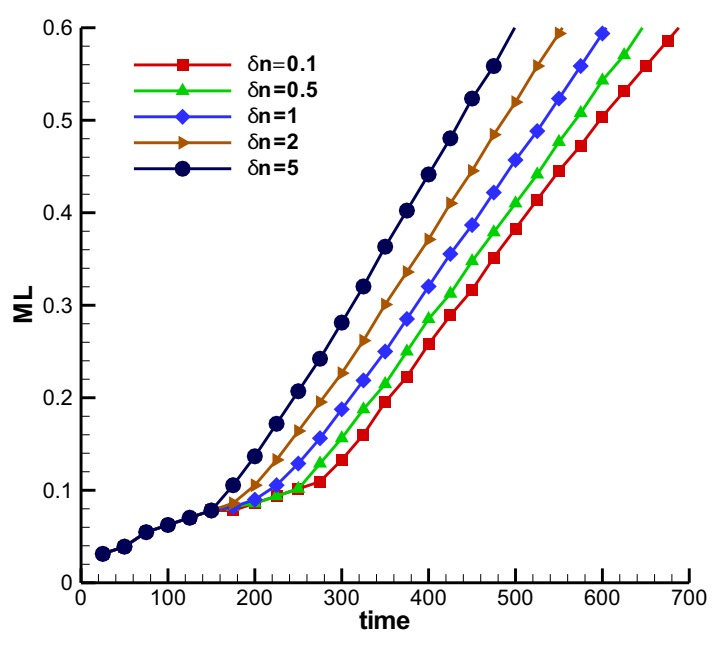

(a) HDC

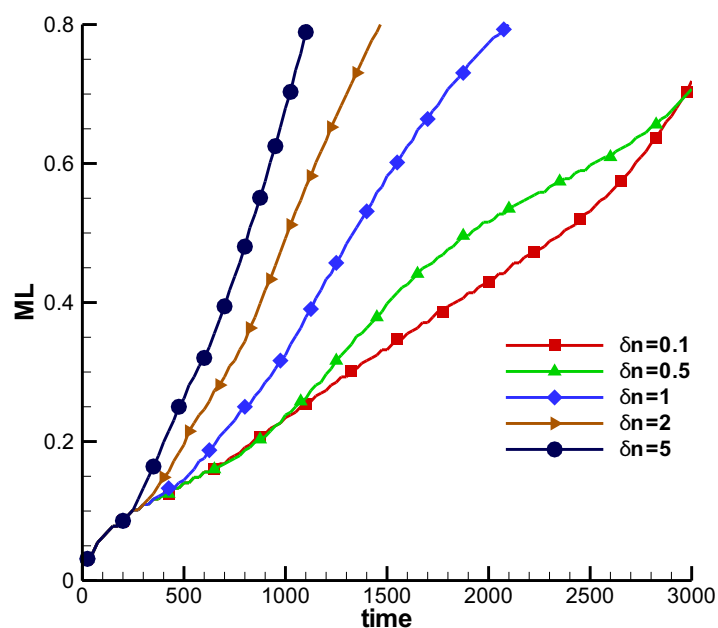

(b) $\mathrm{CDH}$

Figure 4.4: Variation of the Mixing Length with time for different Brownian diffusivities, (a) HDC system and (b) CDH system.

increase of the nanofluid viscosity which reduces the mobility ratio between the fluids and results in the observed attenuation of the instability. For $\delta_{n}=1$ however, the distribution is more spread-out around the front with a wide region of small NP concentrations that contributes to the growth of the instability. These conclusions about the distribution of the NPs concentrations are confirmed by the corresponding plots of $C_{n, a v}$. Furthermore, it is worth noting from Fig. 4.5 that the maximum average NP accumulation in the CDH system starts to decrease after some time, however its location is systematically receding from the front and tends to move upstream.

Fig. 4.5 also reveals the development of localized regions of high NP concentrations in the $\mathrm{CDH}$ case for $\delta_{n}=1$, where NPs in the upstream tend to accumulate in the concave regions between the backward growing fingers (see Fig. 4.5b in the $\mathrm{CDH}$ case). A close examination of the corresponding streamlines presented in Fig. 4.6, reveals that as a result of the convective flow where counter-rotating dipoles develop, fingers grow backward and at the same time NPs are entrained into the space between the growing fingers. This results in the development of regions with large localized concentrations of NPs.

The intensive accumulation of NPs in the narrow band at the front in the CDH system 

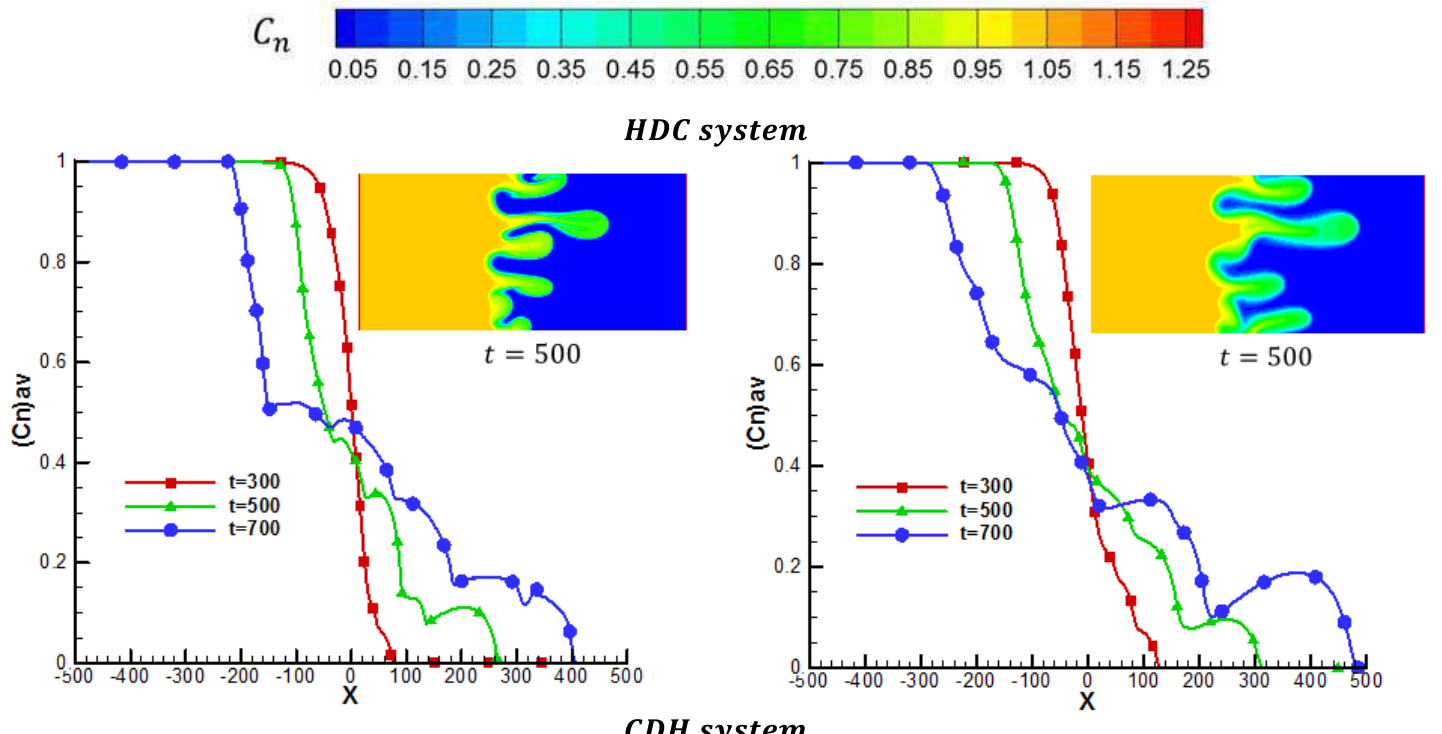

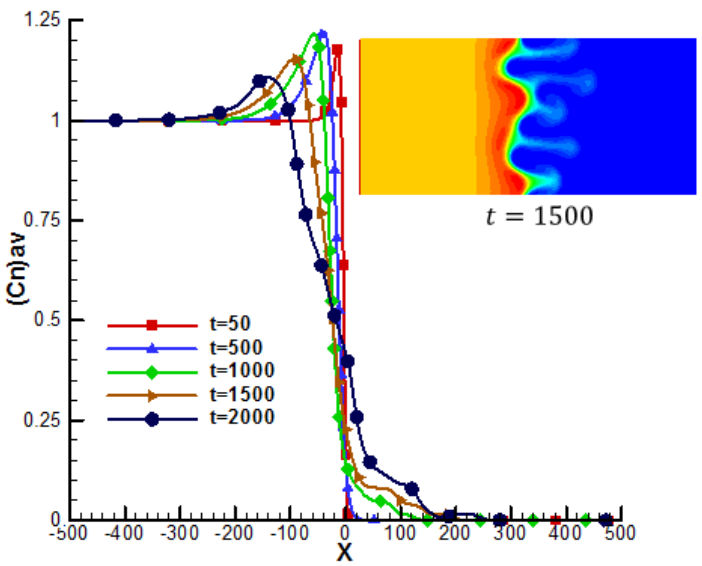

(a) $\delta n=0.1$

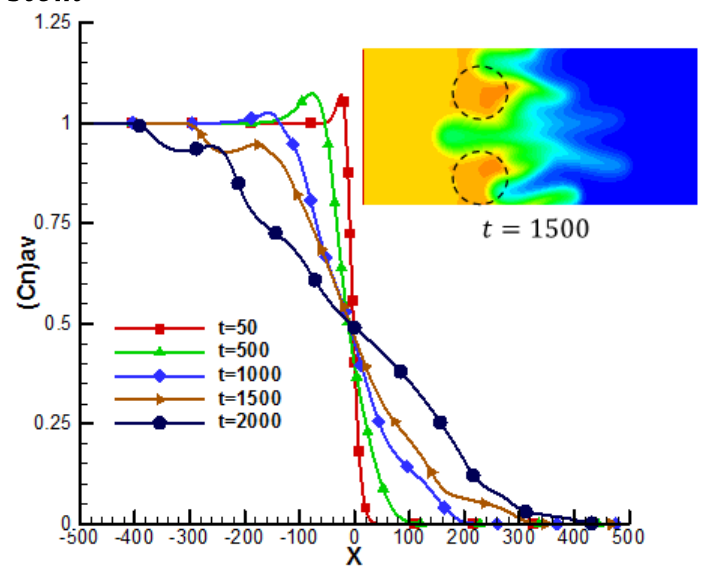

(b) $\delta_{n}=1$

Figure 4.5: Variation of the transversely averaged NP concentration along with contours of $C_{n}$ for both HDC and CDH systems, (a) $\delta_{n}=0.1$ and (b) $\delta_{n}=1.0$ 


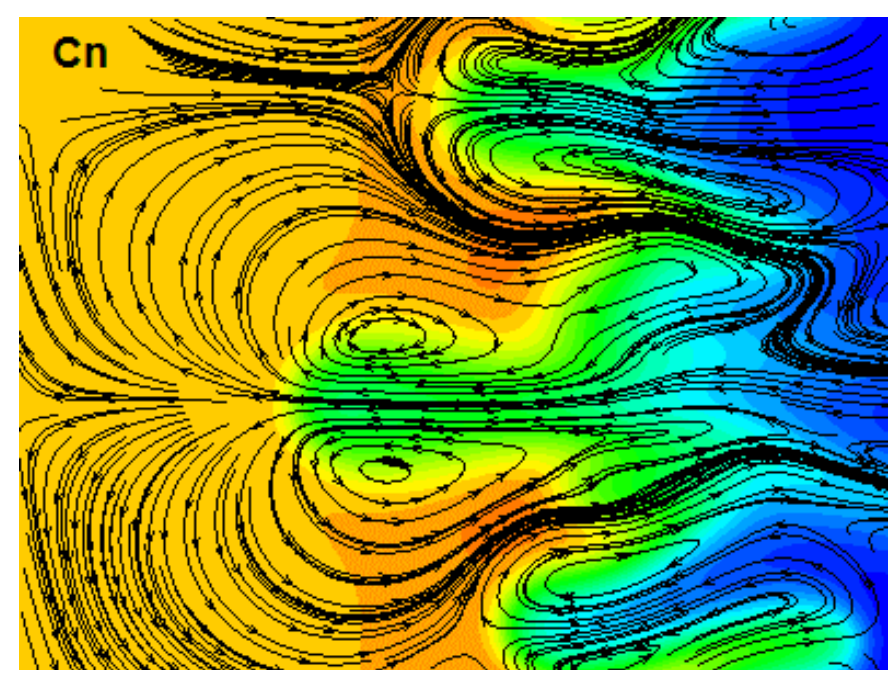

Figure 4.6: A closer view of the contour of $C_{n}$ illustrated in Fig. 4.5 b. The accumulation of NPs in the region between the backward growing fingers is the result of the counter-spinning dipoles $\left(\delta_{n}=1\right)$.

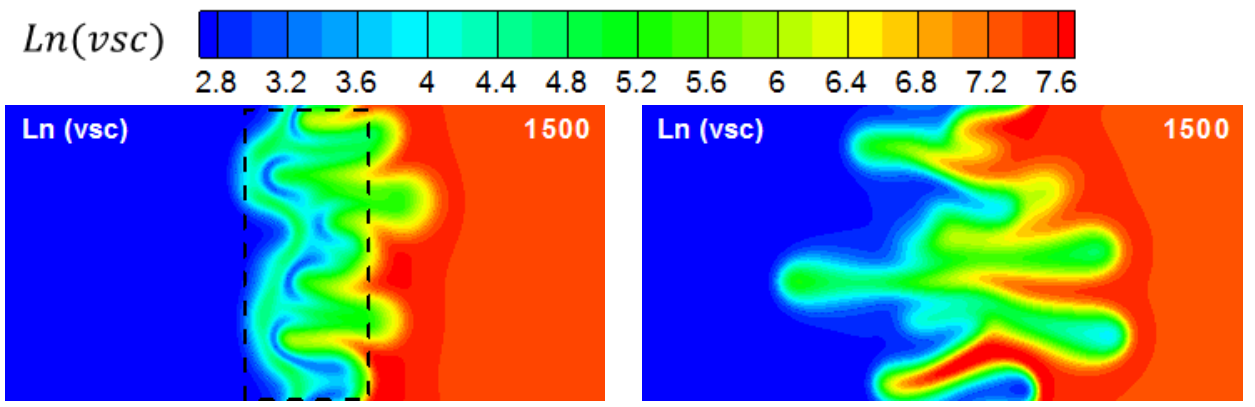
(a) $\delta_{n}=0.1$
(b) $\delta_{n}=1$

Figure 4.7: Viscosity contours for two values of Brownian diffusivity in the CDH system, (a) $\delta_{n}=0.1$ and $(\mathrm{b}) \delta_{n}=1.0$

with $\delta_{n}=0.1$ leads to the creation of stable regions (negative viscosity gradient) upstream of the front. The viscosity distribution depicted in Fig. 4.7 clearly illustrates the local regions of stable flow where a large viscosity fluid displaces a low viscosity one. The development of local stable spots leads to a situation where the instabilities cannot grow easily as already observed in Fig. 4.3a.

To further investigate this, the viscosity distribution is presented along with the streamlines in Fig. 4.8. Four representative regions with specific features have been identified and are labeled in the figure. A relatively high viscosity region 1, which is the consequence of 


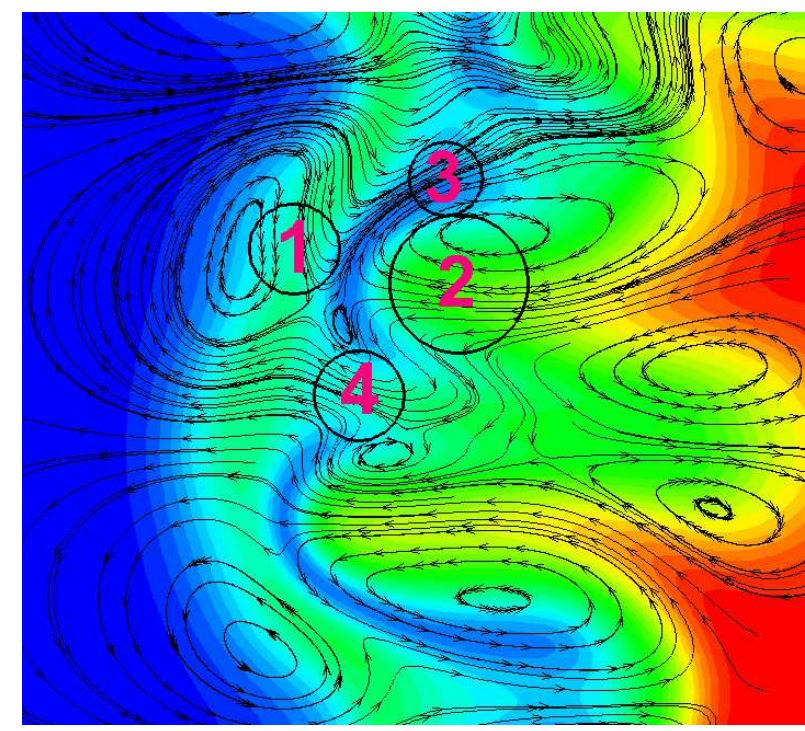

Figure 4.8: A closer view of the viscosity contour depicted in Fig. 4.7a along with the streamlines.

the accumulation of NPs, and region 2 sandwich a lower viscosity region 3. Due to this configuration, the less viscous (more mobile) fluid in region 3 experiences resistance from region 2 to flow downstream and from region 1 to flow upstream. As a result, region 3 acts as a barrier to the flow while the identified region 4 offers a low resistance path to the flow. Due to this barrier, the fluid in region 1 cannot easily flow downstream to feed both forward and backward fingers. The fluid in region 2 experiences the same resistance to flow upstream resulting in the suppression of backward extending fingers. Although part of the fluid can flow downstream through the low resistance region 4, the forward fingers are also unable to grow since, first the extent of the low resistance paths is very small as the stable spots are dispersed in the channel width, and second the mobility ratio between the fluids is reduced because of the presence of small diffusing NPs. This explains why the initiated instabilities are hardly able to grow in the case $\delta_{n}=0.1$. The larger value $\delta_{n}=1$ results in the weakening or even disappearance of the upstream stable spots where the fluid does not experience resistance to backward. Furthermore, due to the diffusion of NPs, the mobility ratio between the fluids increases resulting in a relatively easier growth of forward fingers. Accordingly, fingers growth is stronger compared to the case $\delta_{n}=0.1$. 


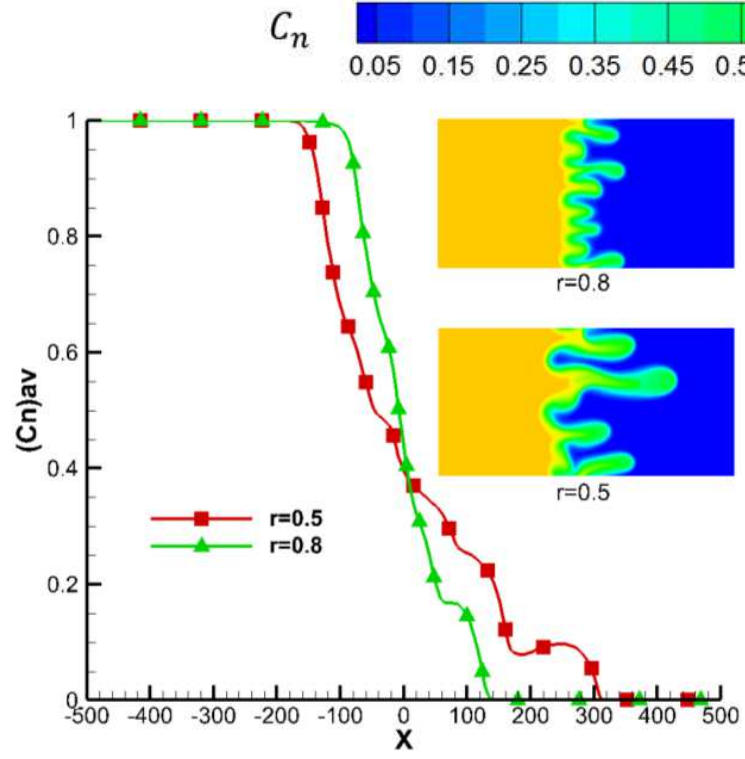

(a) HDC

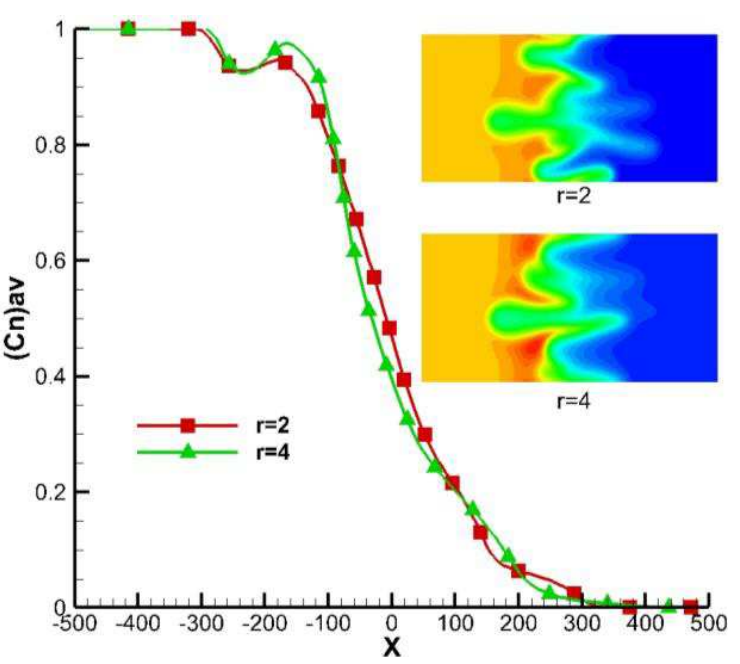

(b) $\mathrm{CDH}$

Figure 4.9: Variation of the transversely averaged NP concentration with insets of NP concentration contours at different temperature ratios for (a) HDC system $(t=500)$ and (b) CDH system $(t=1500)$.

Fig. 4.9 depicts the effects of the temperature ratio $r$ on the NPs distribution and the corresponding variation of $C_{n, a v}$ along the channel. It is clear that increasing the temperature difference of the fluids at constant Brownian diffusivity leads to more downstream migration of NPs in the HDC system and higher upstream accumulation of the NPs in CDH system, similar to the reported effects of $\delta_{n}$. Clearly temperature gradients in NP-laden systems have dramatic effects on the instability. One may therefore infer that Brownian diffusion is not the only mechanism responsible for the observed trends, and heat related mechanisms may be an important factor. Since these trends were not reported in NP-free flows, it is natural to suspect that thermophoresis which is responsible for the transport of NPs as a result of temperature gradients, is playing a major role in the dynamics of the flow.

\subsubsection{Thermophoresis}

Thermophoretic effects are examined by analyzing results for different values of the thermophoretic diffusivity $\delta_{T}$ at constant temperature ratio $r$. It should be noted that as $\delta_{T}$ 


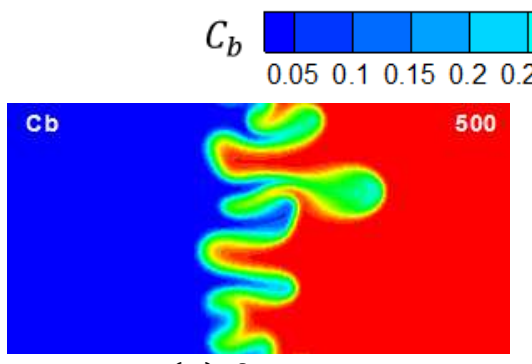

(a) $\delta_{T}=0$
500

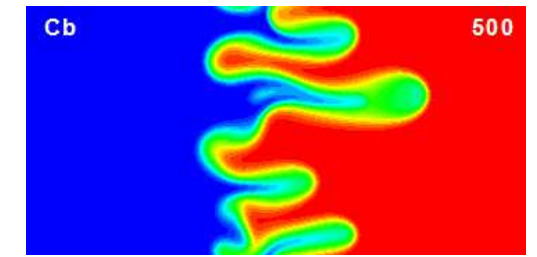

(b) $\delta_{T}=3$

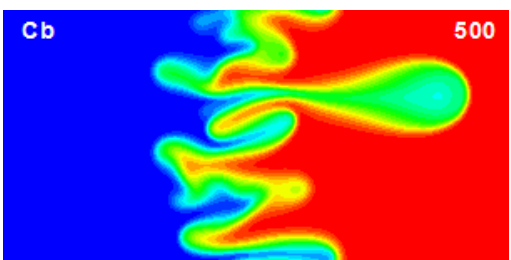

(c) $\delta_{T}=10$

Figure 4.10: Contours of $C_{b}$ for different thermophoretic diffusivities in the HDC system.

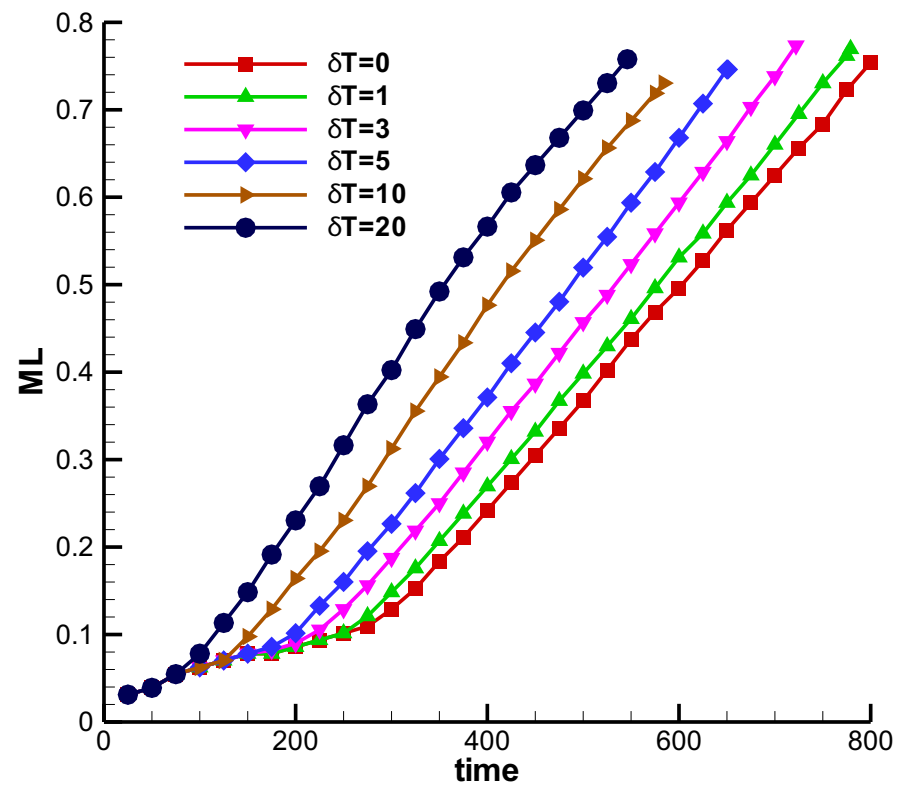

Figure 4.11: Variation with time of the Mixing length for different thermophoretic diffusivities in the HDC system.

increases, the thermophoretic velocity of the NPs increases and vice versa.

Fig. 4.10 shows contours of $C_{b}$ for different values of the thermophoretic diffusivity in the HDC system. It is observed that higher values of $\delta_{T}$ lead to a more unstable flow. The quantitative analysis also validates this conclusion, where for instance according to Fig. 4.11, the system with higher values of $\delta_{T}$ has a larger ML. This increase in the instability corresponds to the local enhancement in the viscosity contrast at the interface as a result of the migration of NPs downstream at higher $\delta_{T}$.

Fig. 4.11 reveals that as $\delta_{T}$ increases the shift from the dispersion regime to the nonlinear fingering regime occurs at earlier times. In the non-linear fingering regime, the fingers interact 


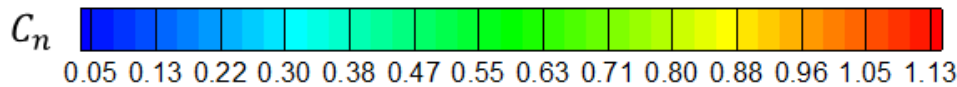

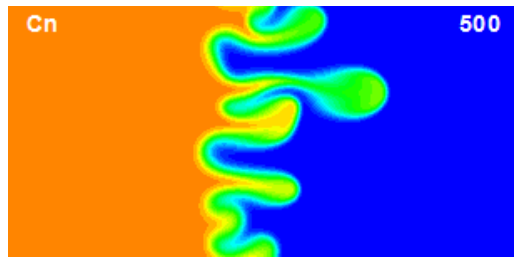

(a) $\delta_{T}=0$

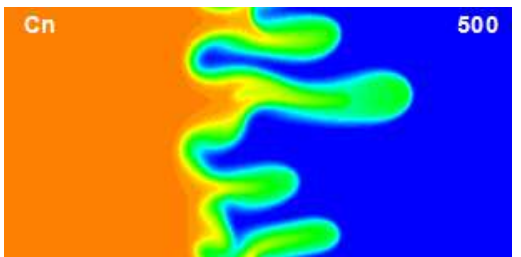

(b) $\delta_{T}=3$

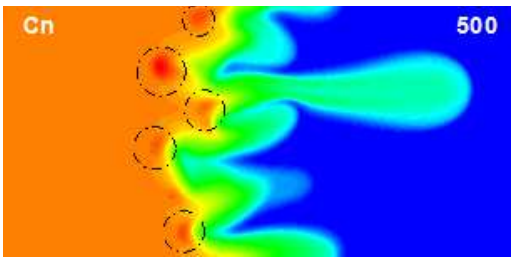

(c) $\delta_{T}=10$

Figure 4.12: Contours of $C_{n}$ for different thermophoretic diffusivities in the HDC system.

closely with each other and the ML grows linearly with time. Furthermore, for the set of parameters considered, the rate of growth of the ML is virtually independent of $\delta_{T}$. One must note that this linear growth with time in the convective regime is typically observed in constant viscosity ratios and large Péclet number flows where the ML growth rate does not depend on dispersion $[22,27,30,97]$. However, since thermophoresis is not a pure diffusive phenomenon, a close analysis of the flow revealed another important factor that can affect the ML growth rate in the non-linear regime, namely the development of non-monotonic viscosity distributions due to the NP migration. In particular we found that the ML growth deviates from the linear regime and also becomes dependent on the thermophoretic diffusivity for large enough NP viscosity ratios, $R_{n}$ as long as the Péclet number is not very large. This effect may be observed in both $\mathrm{HDC}$ and $\mathrm{CDH}$ systems but it is more prominent in the latter case.

An examination of contours of NP concentration depicted in Fig. 4.12 reveals that at large enough values of the thermophoretic diffusivity, localized regions with accumulation of NPs can actually develop in the flow which move upstream with the backward growing fingers (see result for $\delta_{T}=10$ ). This trend was found to be stronger for even larger values of the thermophoretic diffusivity and the nanofluid viscosity. This unexpected trend will be discussed later below.

Different trends were obtained in the $\mathrm{CDH}$ case. The concentration contours of Fig. 4.13 show that unlike the HDC system, increasing $\delta_{T}$ tends now to attenuate the instability. 


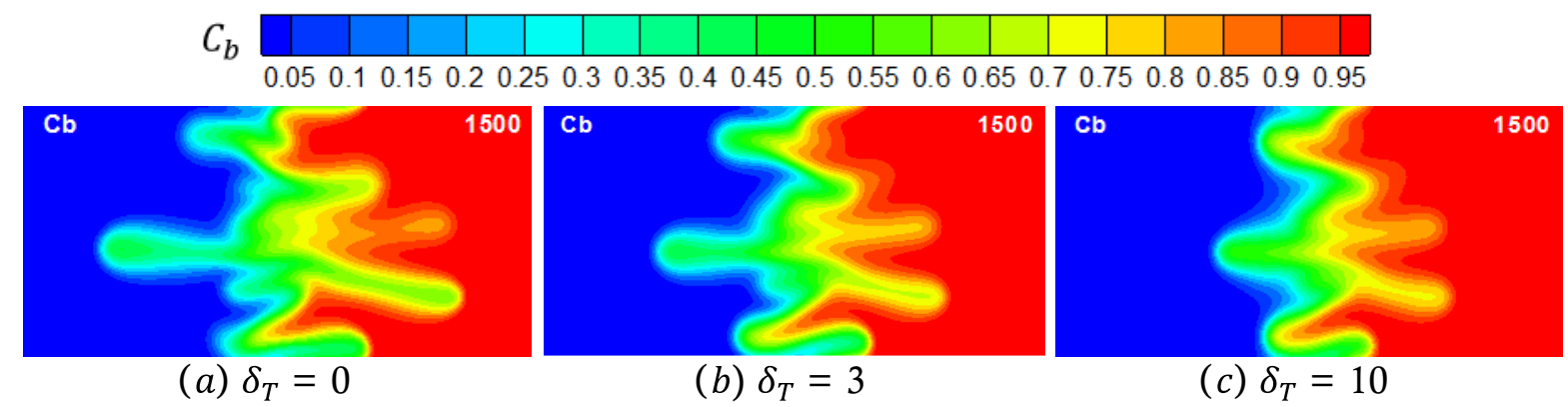

Figure 4.13: Contours of $C_{b}$ for different thermophoretic diffusivities in the CDH system.

In particular backward growing fingers that tend to extend upstream in the absence of thermophoretic effects, are noticeably inhibited for $\delta_{T}=10$. To understand the reason of this attenuation, the distribution of the NP concentration and of the viscosity are analyzed (Fig. 4.14). It is clear that increasing $\delta_{T}$ leads to the accumulation of NPs in the upstream part of the flow, such that the larger the value of $\delta_{T}$ the more the NP accumulation. The upstream NP accumulation also affects the presence of NPs downstream. Specifically, as $\delta_{T}$ increases, the larger accumulation of NPs in the upstream part of the flow implies lower downstream concentration. At higher values of $\delta_{T}$ where the accumulation of NPs is more intense, a local increase in the viscosity is expected. The new distribution of NPs leads to the creation of locally stable upstream regions and hampers the growth of backward developing fingers. This stable position is clearly identified in the viscosity distribution for $\delta_{T}=10$ (Fig. 4.14). Further analysis of the transverse average NP concentration $\left(C_{n, a v}\right)$ which is not illustrated here, indicates that as time passes, the location of $\left(C_{n, a v}\right)_{\max }$ is receding from the front and its value starts to decreases after some time, similar to our discussion of this trend in Fig. 4.5 for the CDH system.

Further increase of $\delta_{T}$ results in another trend in terms of the forward growing fingers. Fig. 4.15 shows the contours of $C_{b}, C_{n}$ and of the viscosity for $\delta_{T}=20$. As expected the NP accumulation is intensified and the backward growth of fingers is more obstructed. However, counter-intuitively, forward developing fingers are actually growing faster, ultimately resulting in the increase of the ML (see Fig. 4.16). This reveals two distinct regimes, whereby 


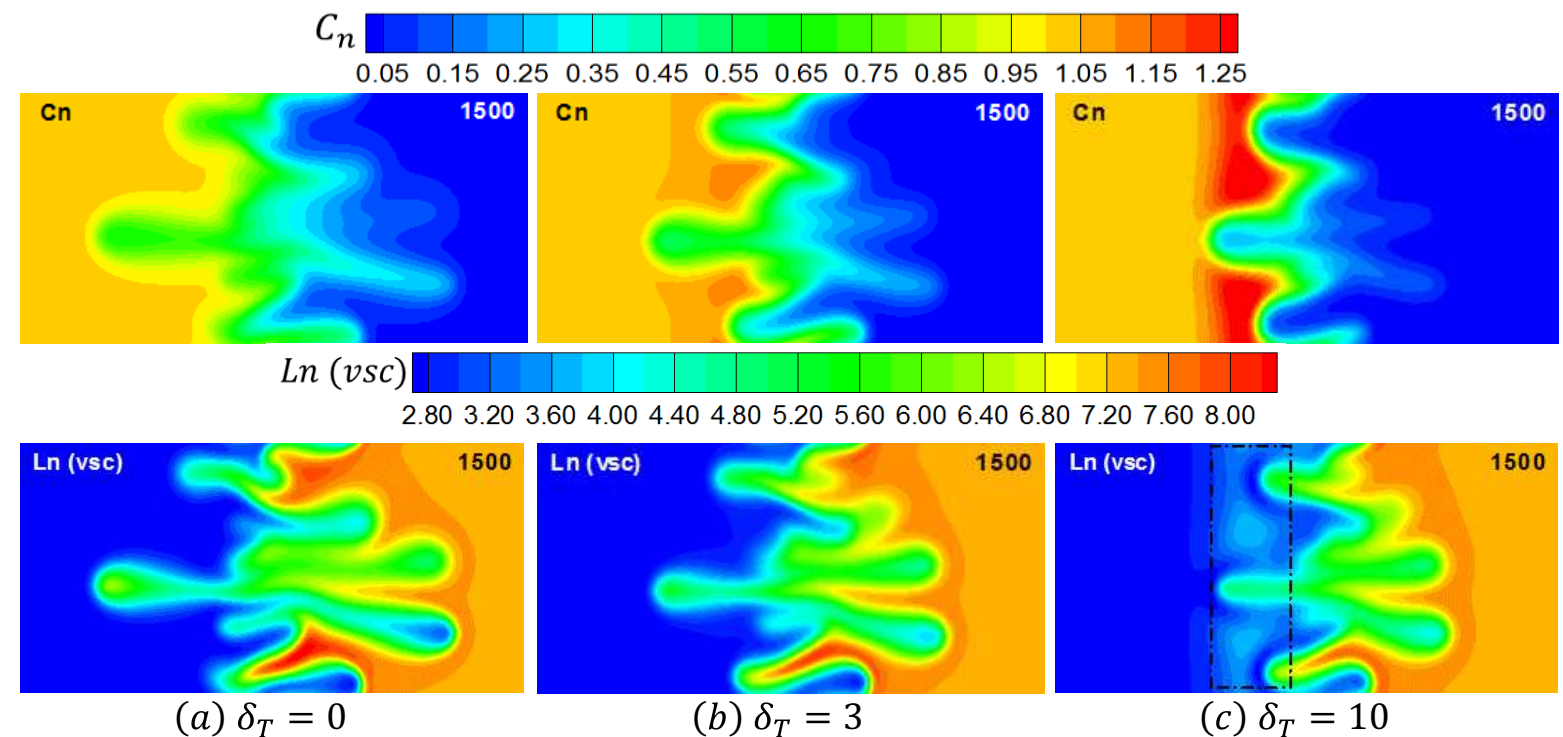

Figure 4.14: Contours of $C_{n}$ and viscosity for different thermophoretic diffusivity in the CDH system.
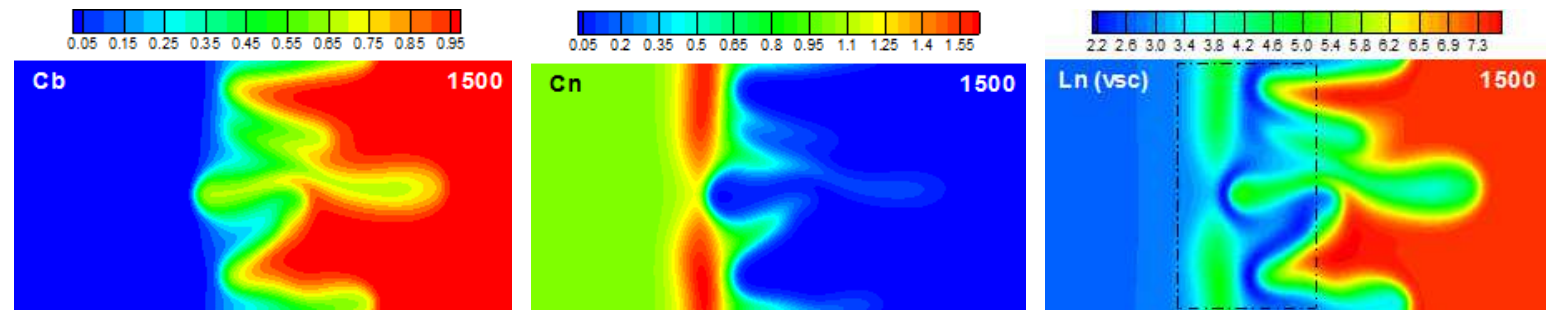

Figure 4.15: Contours of concentrations and viscosity in the CDH system for $\delta_{T}=20$.

initially the ML increases with increasing $\delta_{T}$, followed by a reverse of the trend beyond a critical value of $\delta_{T} \approx 10$. It must however be noted that the conclusions regarding higher accumulations of NPs at the front and the creation of strong stable regions leading to the suppression of backward instabilities, are still valid for higher $\delta_{T}$. Furthermore, the results indicate that generally the $\mathrm{CDH}$ system is less unstable in the presence of thermophoresis in comparison to the case where thermophoresis is absent $\left(\delta_{T}=0\right)$.

To understand the tendency for a faster downstream growth of fingers at higher $\delta_{T}$, viscosity contours for $\delta_{T}=20$ along with the streamlines, are presented in Fig. 4.17. First, it must be mentioned that the reverse flow which is observed in all miscible displacements is driven by convection rather than dispersion, such that the fluid failing to flow through the 


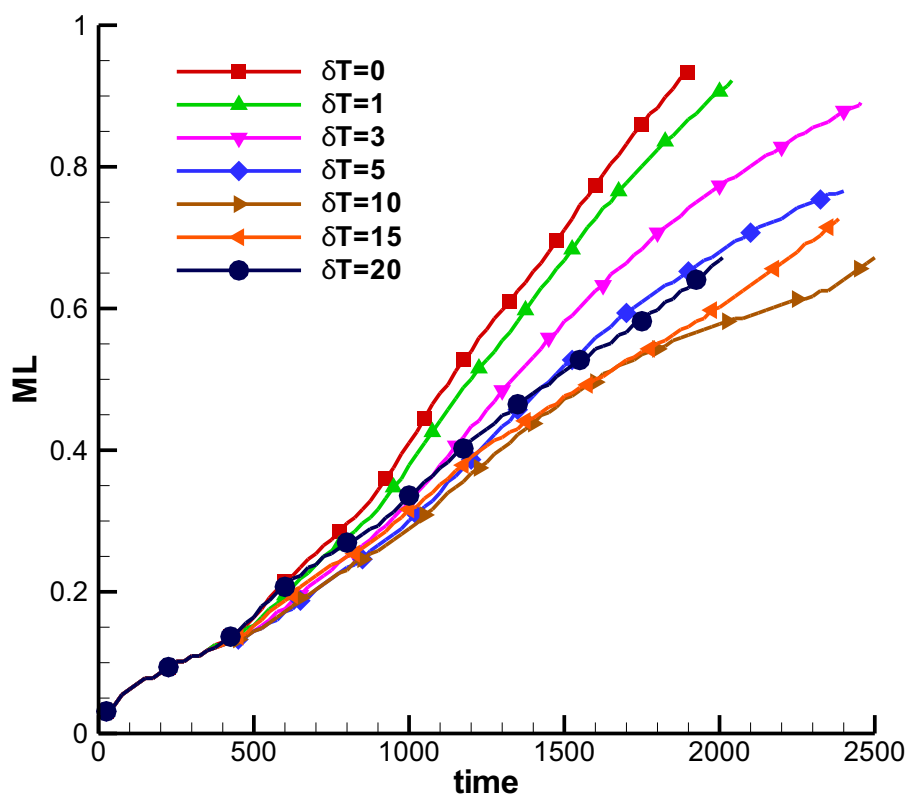

Figure 4.16: Variation with time of the Mixing Length for different thermophoretic diffusivities in the $\mathrm{CDH}$ system.

low resistance path created by the forward fingers, tends to flow backward [70]. Along the same arguments used in the interpretation of the results in Fig. 4.8, at large values of $\delta_{T}$ the backward fingers encounter a region of strong resistance to grow, that arises from the local accumulation of NPs. As a result, the fluid tends instead to return to flow downstream and contributes to the further growth of forward developing fingers. The streaming of the fluid to the forward extending finger is coupled with another effect. As one can notice from Fig. 4.15, the concentration of NPs downstream is reduced as a result of their larger accumulation at the front. The ensuing depletion of NPs especially inside the forward developing fingers leads to an increase of the viscosity contrast inside and outside these fingers. These coupled effects explain the observed faster growth of forward developing fingers and lead to the larger ML.

We will now focus on analyzing the mechanisms of NP accumulation in both HDC and $\mathrm{CDH}$ systems. First, the distributions of the base state characteristics in the HDC case where an unexpected trend was reported, are examined. Fig. 4.18 depicts the base state concentration of NPs at different values of $\delta_{T}$ along with a representative variation of $\frac{\partial \bar{\theta}}{\partial x}$ and 


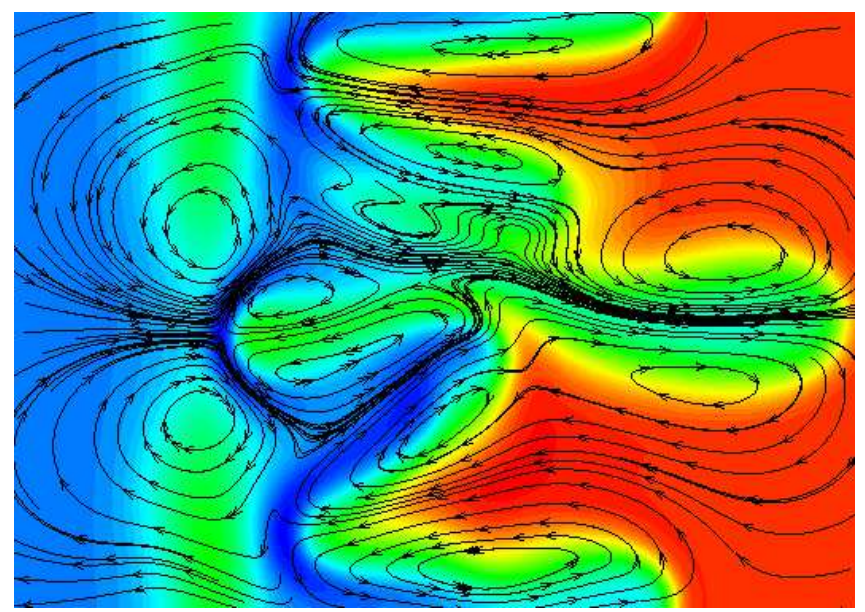

Figure 4.17: The closer view of the viscosity contour of Fig. 4.15 along with the streamlines, $\delta_{T}=20$.

$\frac{\partial^{2} \ln \bar{\theta}}{\partial x^{2}}$. In this figure, $\frac{\partial \bar{\theta}}{\partial x}$ varies from zero to negative values with its absolute maximum at the center of the channel. Furthermore, $\frac{\partial^{2} \ln \bar{\theta}}{\partial x^{2}}=0$ at both ends, with a local positive maximum value at $x>0$ and a local negative minimum value at $x<0$. It is observed that the NP front advances in the channel as $\delta_{T}$ increases. In addition, for small $\delta_{T}, \mathrm{NP}$ concentration decreases monotonically along the channel. However, as $\delta_{T}$ increases, an inflection point appears in the profile of $\bar{C}_{n}$ after a critical value of the thermophoretic diffusivity $\left(\delta_{T_{c r}}\right)$. Further increase of $\delta_{T}$ results in a non-monotonic concentration profile, exhibiting a local minimum and maximum. The local increase in the profile of $\bar{C}_{n}$ represents the local accumulation of the NPs in the flow. The critical values of $\delta_{T}$ associated with the development of an inflection point in the profile of $\bar{C}_{n}$ is generally a function of all other parameters and time. However, as a general trend it was found that with all other parameters fixed, $\delta_{T_{c r}}$ increases with increasing Brownian diffusivity $\delta_{n}$. This fact is illustrated in a representative plot in Fig.4.19.

These trends can be understood by looking at the equation for the NP base state concentration:

$$
\frac{\partial \bar{C}_{n}}{\partial t}=\delta_{n} \bar{\theta} \frac{\partial^{2} \bar{C}_{n}}{\partial x^{2}}+\delta_{T} \frac{\partial}{\partial x}\left(\frac{1}{\bar{\theta}} \frac{\partial \bar{\theta}}{\partial x}\right) \bar{C}_{n}+\left(\delta_{n}+\frac{\delta_{T}}{\bar{\theta}}\right) \frac{\partial \bar{\theta}}{\partial x} \frac{\partial \bar{C}_{n}}{\partial x}
$$

The rate of change of NP concentration is the result of three terms. The first one 


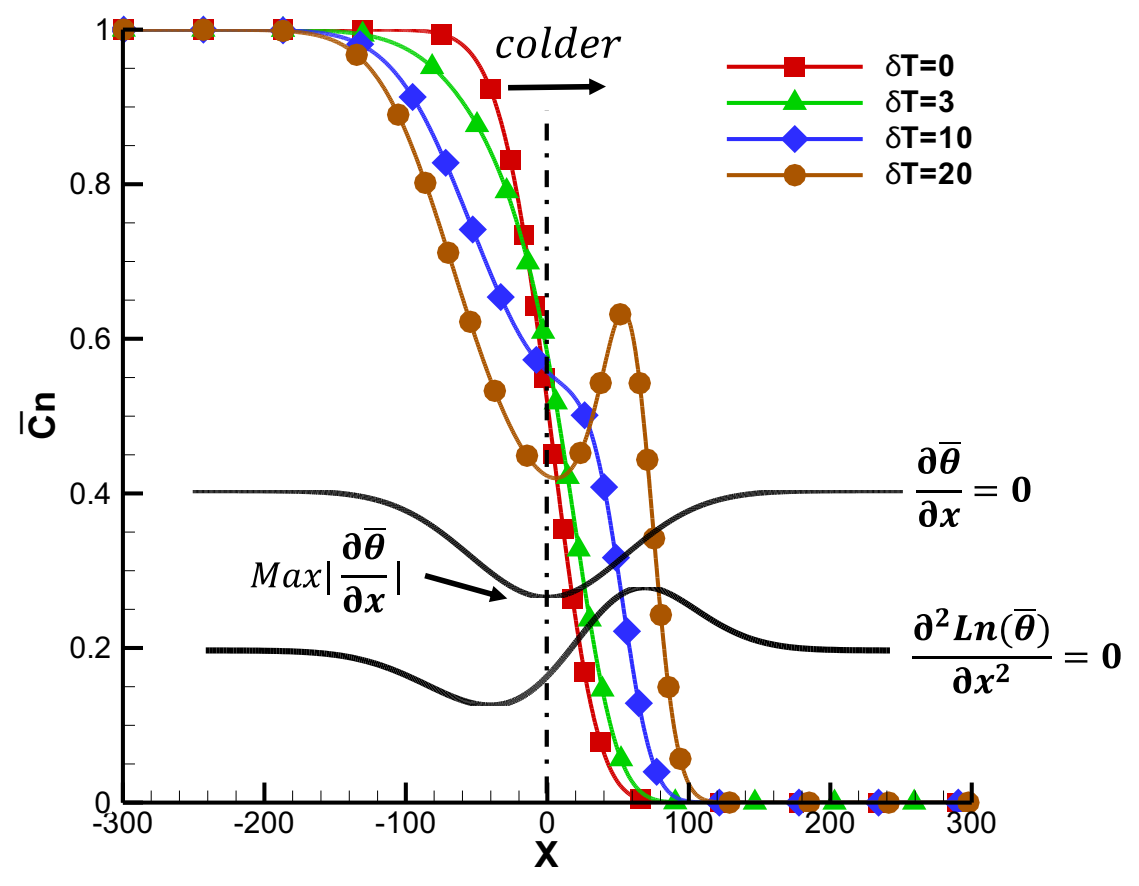

Figure 4.18: Base state NP concentration and a scaled variation of $\frac{\partial \bar{\theta}}{\partial x}$ and $\frac{\partial^{2} \ln \bar{\theta}}{\partial x^{2}}$ along the channel in the HDC system at $t=500$.

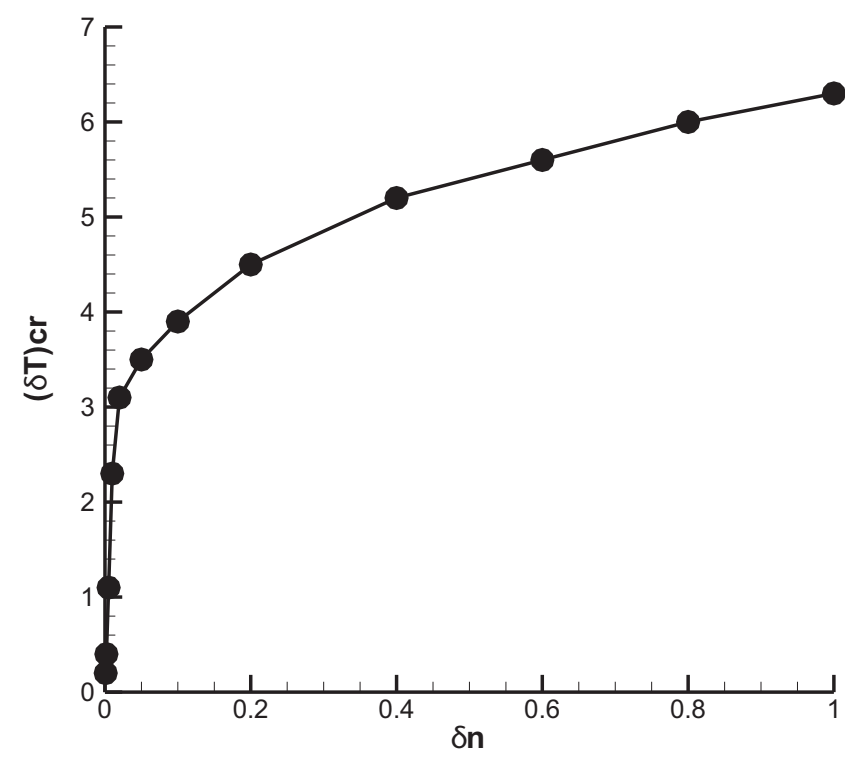

Figure 4.19: A typical variation of $\delta_{T_{c r}}$ with $\delta_{n}$ in the HDC system at $t=500$ and other default parameters. 
corresponds to a diffusion with a temperature dependent diffusivity, while the second term, whose strength is directly proportional to the thermophoretic diffusivity, acts as either a source or a sink term depending on the sign of $\frac{\partial}{\partial x}\left(\frac{1}{\theta} \frac{\partial \bar{\theta}}{\partial x}\right)$. It is clear that since $\frac{\partial}{\partial x}\left(\frac{1}{\theta} \frac{\partial \bar{\theta}}{\partial x}\right)=\frac{\partial^{2} \ln \bar{\theta}}{\partial x^{2}}$ is not constant, the strength of the source/sink term varies in the channel. The last term, can be considered as a convective term with an effective velocity that depends on the temperature gradient and the Brownian and thermophoretic diffusivities. It can be also regarded as the difference of the NP frontal speed compared to that of the solutal/thermal front in the case $\lambda=1$ and negligible thermophoretic and Brownian effects in the energy equation (see section 4.4.4). The expression of this difference in the fronts' speeds at $x=0$ is:

$$
u_{r e}=\frac{1-r}{2 \sqrt{\pi t L e}}\left(\delta_{n}+\frac{2 \delta_{T}}{r+1}\right)
$$

Clearly $u_{r e}=0$ in the isothermal case $\left(r=1, \frac{\partial \bar{\theta}}{\partial x}=0\right)$, implying that all fronts travel at the same speed. The expression of $u_{r e}$ also indicates that the higher $L e$, the smaller this speed difference and the ensuing effects on the instability.

This front speed is positive in the HDC case $\left(r<1, \frac{\partial \theta}{\partial x}<0\right)$ implying that the NP front travels ahead of the solutal/thermal front resulting in the downstream transport of NPs. This difference in the fronts' speeds increases with increasing thermophoretic or Brownian diffusivity. As $\delta_{T}$ increases, the downstream transport of NPs towards the cold fluid increases bringing the NPs into a flow region where their convective velocity is essentially zero $\left(\frac{\partial \bar{\theta}}{\partial x} \rightarrow 0\right)$. Therefore, the NPs in the region with larger absolute temperature gradients are flowing downstream faster $\left(\vec{V}_{t} \propto-\frac{\partial \bar{\theta}}{\partial x}\right)$ while those in the region of cold fluid are moving slowly, leading to the local accumulation of NPs. In fine, the NP front is convected further downstream to a flow region with a high local value of the source term $\delta_{T} \frac{\partial^{2} \ln \bar{\theta}}{\partial x^{2}}>0$ resulting in a local increase in the NP concentration, as already seen in Fig. 4.18 in the case $\delta_{T}=20$.

To complete the discussion the NP accumulation mechanism in the CDH system is examined. Fig. 4.20 depicts the base state NP concentration variation along the channel for 


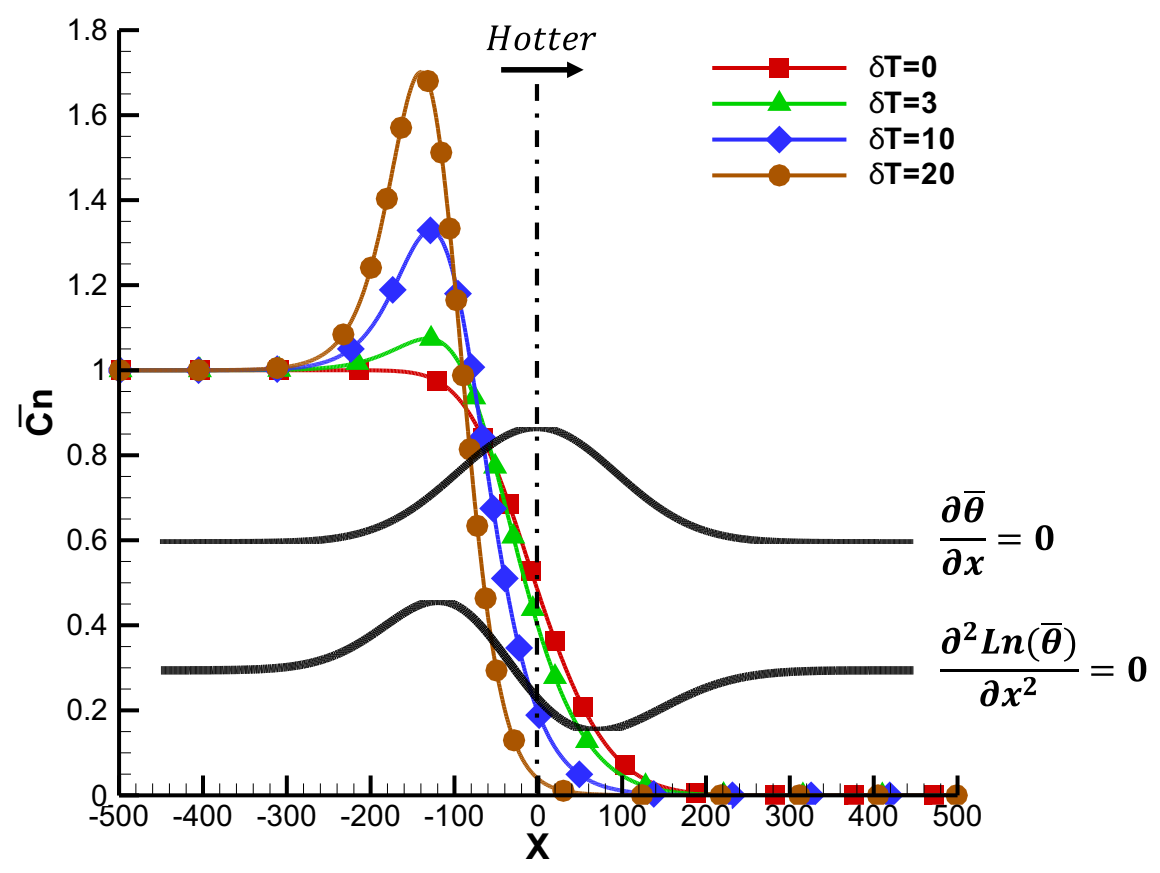

Figure 4.20: Base state NP concentration and a scaled variation of $\frac{\partial \bar{\theta}}{\partial x}$ and $\frac{\partial^{2} \ln \bar{\theta}}{\partial x^{2}}$ along the channel at $\mathrm{t}=1500$ in the $\mathrm{CDH}$ system.

different $\delta_{T}$ with the representative scaled variation of $\frac{\partial \bar{\theta}}{\partial x}$ and $\frac{\partial^{2} \ln \bar{\theta}}{\partial x^{2}}$. Both $\frac{\partial \bar{\theta}}{\partial x}$ and $\frac{\partial^{2} \ln \bar{\theta}}{\partial x^{2}}$ decay to zero at both ends of the domain. However, $\frac{\partial \bar{\theta}}{\partial x}$ has a positive local maximum at the center of the channel, while the variation of $\frac{\partial^{2} \ln \bar{\theta}}{\partial x^{2}}$ exhibits a positive local maximum at $x<0$ and a negative local minimum at $x>0$. The figure shows that as $\delta_{T}$ increases, NPs tend to accumulate at the front and the location of both NP front and $\left(\bar{C}_{n}\right)_{\max }$ move upstream. As in the HDC case, these trends can be understood by examining the NP base state equation, Eq. 4.34. Since $\frac{\partial \bar{\theta}}{\partial x}>0$ in this case, the convective term $\left(\delta_{n}+\frac{\delta_{T}}{\theta}\right) \frac{\partial \bar{\theta}}{\partial x}$ is always positive (or $\left.u_{r e}<0\right)$, implying that the NP front convects more upstream as $\delta_{T}$ increases and leads to a stronger presence of NPs in the upstream region $x<0$, and in turn a weaker presence in the downstream section; $x>0$. However in $x<0$, NPs have different thermophoretic velocities, with stronger backward velocity due to the higher positive $\frac{\partial \bar{\theta}}{\partial x}\left(\vec{V}_{t} \propto-\frac{\partial \bar{\theta}}{\partial x}\right)$ close to $x=0$, and an almost zero thermophoretic velocity far away from the front. As a result, the NPs tend to accumulate at the front and this accumulation intensifies more as $\delta_{T}$ increases.

The accumulation is a result of thermophoresis and is strengthened with $\delta_{T}$, since as 
the thermophoretic diffusivity increases, the source term $\delta_{T} \frac{\partial^{2} \ln \bar{\theta}}{\partial x^{2}}$ gets larger at $x<0$ and also more NPs are convected upstream $\left(\left(\delta_{n}+\frac{\delta_{T}}{\theta}\right) \frac{\partial \bar{\theta}}{\partial x}\right)$. Although an increase of the Brownian diffusivity results in larger NP frontal speed $u_{r e}$ and consequently a stronger upstream transport of NPs, it is also accompanied with stronger Brownian diffusion which explains the observed trend towards smaller NP accumulation as the Brownian effects gets stronger (see Fig. 4.5 for CDH case).

The previous results indicate that Brownian and thermophoretic effects have similar destabilizing effects in the HDC system, where larger values of $\delta_{n}$ or $\delta_{T}$ lead to a more unstable displacement. The effects of these two mechanisms are however different in the $\mathrm{CDH}$ case, where a stronger Brownian diffusion tends to increase the instability while larger thermophoretic effects have actually a stabilizing effect at least in some ranges. In particular a large accumulation of NPs that leads to local stable regions upstream of the front, are observed in the CDH case for smaller $\delta_{n}$ and larger $\delta_{T}$ which hinders the growth of backward developing fingers. These results point to the close interplay of these two effects that can either act synergistically or have counter-effects. In the HDC case, Brownian diffusivity and thermophoresis act together towards the migration of the NPs in the downstream direction, while in the $\mathrm{CDH}$ case where the temperature gradient is opposite to the HDC case, Brownian diffusion tends still to transport NPs downstream of the flow towards the hot fluid while now thermophoresis acts in the opposite direction resisting the downstream migration of the NPs. Accordingly, the counter effects of thermophoresis in the CDH case are more noticeable for smaller $\delta_{n}$ or larger $\delta_{T}$. In the HDC case on the other hand, the two mechanisms have synergistic effects, and larger values of $\delta_{n}$ and/or $\delta_{T}$ lead to the migration of NPs in the downstream direction towards the cold less viscous fluid, resulting in a more unstable displacement. 


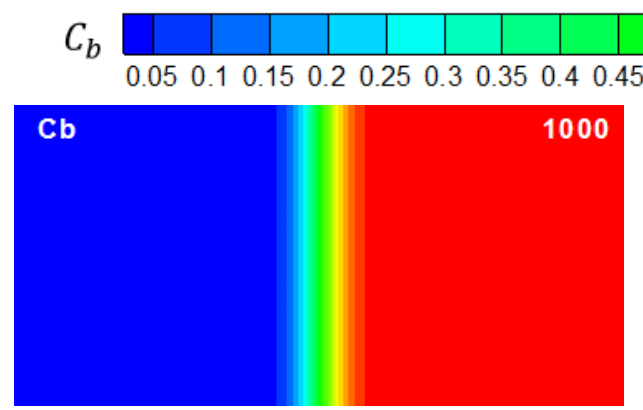

(a) $\delta_{T}=0$
1000

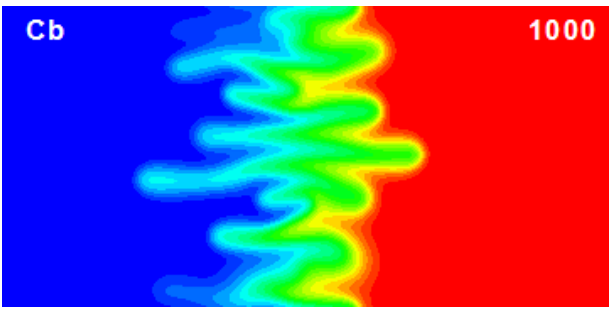

(b) $\delta_{T}=10$

Figure 4.21: Contours of $C_{b}$ in the HDC system with $R_{n}=5$.

\subsubsection{Thermophoretic effects in stable/intinsically stable systems}

The previous results and physical interpretations lead to the natural question of whether thermophoresis effects would destabilize a stable or intrinsically stable system. To answer this question, the instability of both HDC and CDH systems with large nanofluid viscosity ratio, $R_{n}=5$, is investigated in the presence and the absence of thermophoretic effects. Note that such a flow is unstable $\left(R_{b}=3, R_{\theta}=1(\mathrm{HDC}), R_{\theta}=-1(\mathrm{CDH})\right)$ in the absence of NPs.

Fig. 4.21 shows the concentration contours in the HDC system in the presence and absence of thermophoretic effects. It is observed that the system is stable in the absence of thermophoreis $\left(\delta_{T}=0\right)$, while it is unstable otherwise $\left(\delta_{T}=10\right)$. The instability develops as a result of the advancement of the NP front downstream which then leads to the non-monotonicity in viscosity distribution. Therefore, one can extend the enhancement of instability in an already unstable flow to a destabilization one of a stable flow, when one is dealing with the HDC case. The analysis did not show this effect in the CDH system such that the system was stable both in the presence and the absence of thermophoresis.

The problem is now extended to an intrinsically stable system. The choice of $R_{b}=-1$ (more viscous fluid displacing a less viscosus one) represents the intrinsically stable HDC system $\left(R_{\theta}=1\right)$ in the absence of $\operatorname{NPs}\left(R_{n}=0, \epsilon=0\right)$. Note that the default values of the other parameters are still used. Fig. 4.22 reveals that although the addition of NPs further increases the viscosity of the displacing fluid and may lead one to expect the flow stability to 


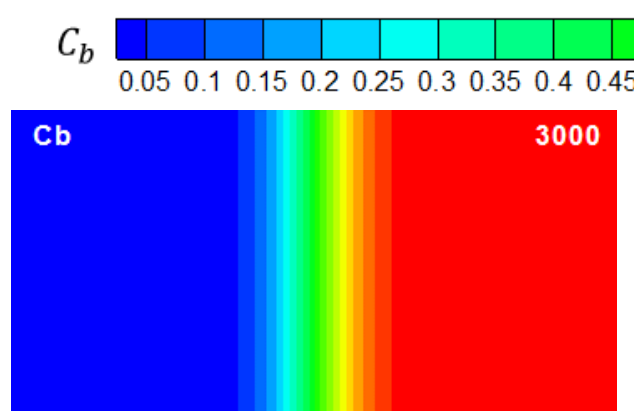

(a) $R_{n}=0$

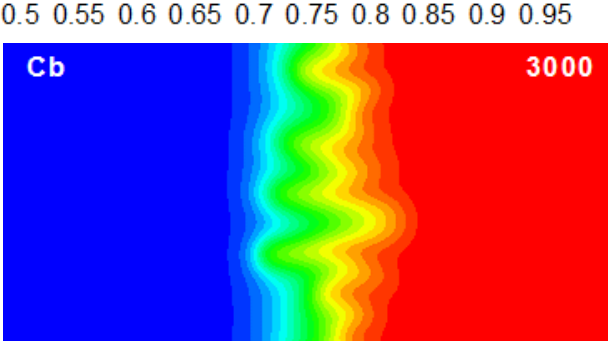

(b) $R_{n}=1, \delta_{T}=20$

Figure 4.22: Contours of $C_{b}$ in the HDC system for $R_{b}=-1$ in (a) the absence, (b) the presence of NPs. Note that $R_{n}$ and $\epsilon$ are set to zero to generate the case without NPs.

be maintained, it is found that the dynamics are changed and fingers start to develop in the flow as a result of thermophoretic effects $\left(\delta_{T}=20\right)$. In other words such a system became unstable only as a result of the addition of NPs and their migration due the already present temperature gradient. This shows that thermophoresis can even make an intrinsically stable HDC system, unstable. The CDH system with $R_{b}=-1$ did not show this effect.

\subsubsection{Thermophoresis and Brownian diffusion in the energy equa- tion}

The previous results and interpretation were based mainly on changes in the distribution of NPs as a result of Brownian and thermophoretic effects. However, these effects are also present in the energy equation and it is legitimate to ask if the dynamics can be explained by an analysis of the contributions of different terms in the energy equation, Eq. 4.15. The last two terms in the right hand side of this equation represent the Brownian and thermophoresis contributions. However, their effects on the instability depend directly on the value of $\epsilon=\frac{C_{n_{0}} \phi\left(\rho c_{p}\right)_{p}}{\left(\rho c_{p}\right)_{m}}$ which has not been addressed yet.

Note that the volumetric heat capacity $\left(\rho c_{p}\right)$ of both solids and liquids are of the order of $10^{6}$. Furthermore, the maximum order of magnitude of the NPs mass/volume fraction and the porosity are typically $10^{-2}$ and $10^{-1}$, respectively. This leads to the conclusion that $O(\epsilon)=O\left(C_{n_{0}} \phi\right)$, or $\epsilon<0.01$. Adopting $\epsilon=0.01$ the contributions of the Brownian 
motion and thermophoresis are analyzed while other parameters are fixed. Examinations of contours and maximum and average values of the diffusive term $L e \nabla^{2} \theta$ and of the combined Brownian-thermophoretic term $\epsilon\left(\delta_{n} \theta \vec{\nabla} C_{n} \cdot \vec{\nabla} \theta+\delta_{T} C_{n} \frac{\vec{\nabla} \theta \cdot \vec{\nabla} \theta}{\theta}\right)$ revealed that the latter term is order of magnitudes smaller than the former, for both HDC and CDH cases. The analysis revealed almost the same conclusion even with $\epsilon=0.1$. This result was found systematically over a wide range of the other flow parameters. It clearly points to the negligible contribution of the Brownian motion and thermophoresis in the energy equation and to the fact that such mechanisms can be actually ignored in the modeling and analysis of the energy effects, at least for the system at hand. It also implies that the main effects of Brownian diffusion and thermophoresis arise from their contribution to the NP transport while their contribution through the energy equation can be safely disregarded.

$$
\frac{\partial \theta}{\partial t}+(\lambda-1) \frac{\partial \theta}{\partial x}+\lambda(\vec{V} \cdot \vec{\nabla} \theta)=L e \nabla^{2} \theta
$$




\section{Chapter 5}

\section{Dynamics of Nano-Catalytic Reactive Flows in Porous Media}

${ }^{1}$ As a result of the increase in use of nano-catalysts in different applications particularly those for enhancing reaction rates in porous media [18, 98-100], and due to the important effects of nano-catalysts on both the properties of the fluids and the reaction rate, miscible VF instabilities of reactive systems will be investigated in the presence of dispersed nanocatalysts in the invading fluid. Specifically, the objective of this study is to analyze the VF instabilities of nano-catalytic systems in the presence of $A+B+n \rightarrow C+n$ reaction which is more prone to create viscosity mismatch compared to the autocatalytic reaction. The analysis will first examine the case of isothermal flows and then focus on non-isothermal conditions that result from the heat of the reaction. This phase of the analysis is important as shall be seen later, the nano-catalysts are subject in particular to thermophoretic effects that have the potential to alter the dynamics of the flow.

\footnotetext{
${ }^{1}$ This chapter is based on the following journal article:

B. Dastvareh, J. Azaiez, "Instabilities of Non-Isothermic Nano-Catalytic Reactive Flows in Porous Media", Physical Review Fluids 4(3)(2019) 034003.
} 


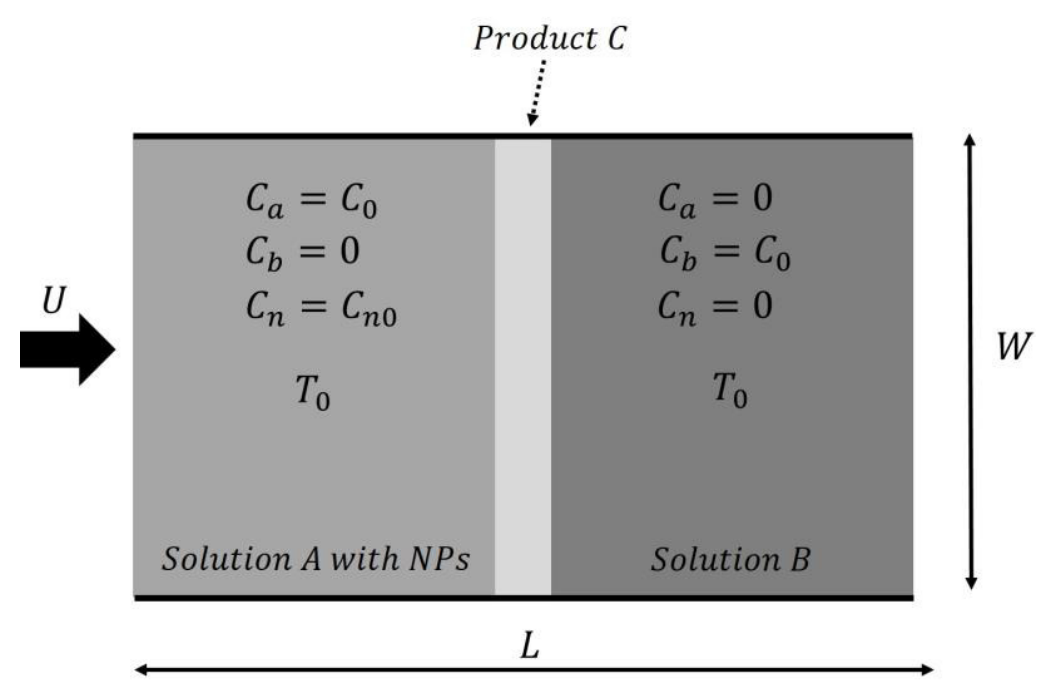

Figure 5.1: Schematic view of the medium

\subsection{Physical problem}

A schematic view of the system is illustrated in Fig. 5.1 representing a homogeneous porous medium or equivalently a Hele-Shaw cell. The channel is initially occupied by a solution $B$ with the initial mass fraction (or volume fraction in ideal solutions) of $C_{0}$ and the viscosity $\mu_{b_{0}}=\mu\left(0, C_{0}, 0,0\right)$. Solution $A$ with the same mass fraction of $C_{0}$ and the viscosity $\mu_{a_{0}}=$ $\mu\left(C_{0}, 0,0,0\right)$ carrying nano-catalysts with initial mass fraction of $C_{n_{0}}$ and the viscosity $\mu_{n_{0}}=$ $\mu\left(0,0,0, C_{n_{0}}\right)$ is injected into the channel at a velocity $U$. As soon as the solutions are in contact, the following reaction takes place:

$$
A+B+n \rightarrow C+n
$$

The viscosity of the chemical product $\mathrm{C}$ at the mass fraction of $C_{0}$ is $\mu_{c_{0}}=\mu\left(0,0, C_{0}, 0\right)$. Before the reaction takes place the system is at temperature of $T_{0}$, which may change after the reaction as a result of the change in the enthalpy, $\Delta H . \Delta H<0$ if the reaction is exothermic and $\Delta H>0$ when it is endothermic. Finally, it is assumed that the fluids are incompressible and the nano-catalysts are in thermal equilibrium with the medium. Henceforth NP notation may refer to either nano-catalysts or nanoparticles. 


\subsection{Problem formulation}

The problem is governed by the following equations representing the conservation of mass, the conservation of momentum in the form of Darcy's law, transport of the components A, B, C, and NPs and finally the conservation of energy:

$$
\begin{gathered}
\vec{\nabla} \cdot \vec{V}_{D}=0 \\
\vec{\nabla} P=-\frac{\mu}{K} \vec{V}_{D} \\
\frac{\partial C_{j}}{\partial t}+\frac{1}{\phi}\left(\overrightarrow{V_{D}} \cdot \vec{\nabla} C_{j}\right)=D_{j} \nabla^{2} C_{j} \pm r_{R} \\
\frac{\partial C_{n}}{\partial t}+\frac{1}{\phi}\left(\overrightarrow{V_{D}} \cdot \vec{\nabla} C_{n}\right)=\vec{\nabla} \cdot\left(D_{n} \vec{\nabla} C_{n}+D_{T} \frac{\vec{\nabla} T}{T}\right)-k_{d e p} C_{n} \\
\frac{\partial T}{\partial t}+\frac{\lambda}{\phi}\left(\vec{V}_{D} \cdot \vec{\nabla} T\right)=\alpha \nabla^{2} T-\frac{\phi \Delta H}{\left(\rho c_{p}\right)_{m}} r_{R}
\end{gathered}
$$

Where $\vec{V}_{D}$ is the Darcy's velocity, $P$ the local pressure, $\mu$ the viscosity and $K$ the intrinsic medium permeability. In Eq. 5.4, $C_{j}=\left(C_{a}, C_{b}, C_{c}\right)$ is the mass/volume (in ideal solutions) fraction of the components, $D_{j}=\left(D_{a}, D_{b}, D_{c}\right)$ the corresponding diffusion coefficient, $\phi$ the porosity and $r_{R}$ is the rate of reaction. $D_{j}$ are assumed to be constant while the cross diffusion and Soret effects are ignored as they are order of magnitude smaller than the principal diffusion effects. A first order dependency on the concentration of the reactants and the NPs is adopted for the rate of reaction, where $r_{R}=k_{R} C_{a} C_{b} C_{n}$. Note that the linear dependency of the reaction rate on the nano-catalysts concentration has been reported in previous experimental studies [101-104]. Moreover, it is assumed that the heat of the reaction is small enough not to affect the dynamics through $k_{R}$ which will be considered constant. $D_{n}$ and $D_{T}$ in Eq. 5.5 represent the Brownian and thermophoretic diffusion coefficients of NPs while $k_{d e p}$ is the deposition rate following the widely applied colloid filtration model [67]. Finally, $\lambda=\frac{\phi\left(\rho c_{p}\right)_{n f}}{\left(\rho c_{p}\right)_{m}}$ is the thermal lag coefficient [42] where the subscripts nf and $\mathrm{m}$ refer to the nanofuid and the medium respectively, $\alpha=\frac{k_{m}}{\left(\rho c_{p}\right)_{m}}$ thermal diffusivity, $\rho c_{p}$ the volumetric heat capacity and $k$ the thermal conductivity. Brownian 
and thermophoresis diffusion coefficients are in general not constant. In particular based on Einstein equation $\left(D_{n}=\frac{k_{B} T}{3 \pi \mu_{b f} d_{p}}\right)$ it is assumed that $D_{n}$ varies linearly with temperature while following Piazza and Parola [93] and Buongiorno [84], $D_{T}$ in Eq. 5.5 is a linear function of $C_{n}$. Assuming $D_{n}=D_{n 0} \frac{T}{T_{0}}$ and $D_{T}=D_{T 0} \frac{C_{n}}{C_{n 0}}$ [105] and incorporating constant $K$ into the viscosity definition, the equations are then made dimensionless. Accordingly, the length, time and pressure are scaled with $\frac{D_{a} \phi}{U}, \frac{D_{a} \phi^{2}}{U^{2}}, D_{a} \phi \mu_{a}$, viscosity with $\mu_{a}$, velocity with $U, C_{j}$ with $C_{0}$ and $C_{n}$ with $C_{n 0}$. Finally the dimensionless temperature is defined as $\theta=\frac{T-T_{0}}{T_{0} H_{R}}$, where $H_{R}=\frac{-\phi \Delta H C_{0}}{T_{0}\left(\rho c_{p}\right)_{m}}=\frac{\Delta T}{T_{0}} . H_{R}$, which is assumed to be small $\left(H_{R}<<1\right)$ and does not lead to any fluid phase changes, represents the temperature changes as a result of the reaction. Finally, the equations are formulated in a reference frame moving with the velocity $U$. The equations are then in the following form:

$$
\begin{gathered}
\vec{\nabla} \cdot \vec{V}=0 \\
\vec{\nabla} P=-\mu(\vec{V}+\vec{i}) \\
\frac{\partial C_{j}}{\partial t}+\vec{V} \cdot \vec{\nabla} C_{j}=\delta_{j} \nabla^{2} C_{j} \pm D a C_{a} C_{b} C_{n} \\
\frac{\partial C_{n}}{\partial t}+\vec{V} \cdot \vec{\nabla} C_{n}=\delta_{n} \vec{\nabla} \cdot\left(\left(1+H_{R} \theta\right) \vec{\nabla} C_{n}\right)+\delta_{T} \vec{\nabla} \cdot\left(C_{n} \vec{\nabla} \operatorname{Ln}\left(1+H_{R} \theta\right)\right)-D a_{d e p} C_{n} \\
\frac{\partial \theta}{\partial t}+(\lambda-1) \frac{\partial \theta}{\partial x}+\lambda(\vec{V} \cdot \vec{\nabla} \theta)=\operatorname{Le} \nabla^{2} \theta+\operatorname{sgn}\left(H_{R}\right) D a C_{a} C_{b} C_{n}
\end{gathered}
$$

In these equations all the variables are dimensionless, where:

$$
\begin{gathered}
\delta_{j}=\frac{D_{j}}{D_{a}}, \quad \delta_{n}=\frac{D_{n 0}}{D_{a}}, \quad \delta_{T}=\frac{D_{T_{0}}}{D_{a} C_{n_{0}}}, \quad L e=\frac{\alpha}{D_{a}} \\
D a_{d e p}=\frac{k_{d e p} D_{a} \phi^{2}}{U^{2}}, \quad D a=\frac{k D_{a} \phi^{2} C_{0} C_{n 0}}{U^{2}}
\end{gathered}
$$

Where $\delta_{j}, \delta_{n}, \delta_{T}$ are the solute, Brownian and thermophoretic diffusivities respectively, Da Damköhler number, Le Lewis number and $D a_{d e p}$ the dimensionless deposition rate. The positive sign in Eq. 5.9 is used for component $\mathrm{C}$ while the negative sign is used for components A and B. Furthermore, $H_{R}>0$ and accordingly $\operatorname{sgn}\left(H_{R}\right)=+1$ in the exothermic reaction 
while $H_{R}<0, \operatorname{sgn}\left(H_{R}\right)=-1$ in the case of endothermic one. With the introduced scalings, the dimensions of the domain are $\left(-\frac{P e}{2}, \frac{P e}{2}\right)$ in the $x$ direction and $\left(0, \frac{P e}{A_{s}}\right)$ in the y direction, where $P e=\frac{U L}{\phi D_{a}}$ is the Péclet number and $A_{s}=\frac{L}{W}$ is the domain aspect ratio. In the moving reference, the concentrations of A, B and NPs obey the zero flux condition at the x-boundaries while the velocity, $C_{c}$ and $\theta$ are zero. In addition, periodic boundary conditions are used in the y-direction. The model is completed by adopting the following widely used exponential viscosity-concentration-temperature relationship [22, 42]:

$$
\mu=\exp \left(R_{b} C_{b}+R_{c} C_{c}+R_{n} C_{n}+R_{\theta} \theta\right)
$$

Where $R_{b}, R_{c}, R_{n}$ and $R_{\theta}$ are the mobility ratios defined as:

$$
R_{b}=\ln \left(\frac{\mu_{b_{0}}}{\mu_{a_{0}}}\right)_{T_{0}}, \quad R_{c}=\ln \left(\frac{\mu_{c_{0}}}{\mu_{a_{0}}}\right)_{T_{0}}, \quad R_{n}=\ln \left(\frac{\mu_{n_{0}}}{\mu_{a_{0}}}\right)_{T_{0}}, \quad R_{\theta}=\ln \left(\frac{\left.\mu_{T_{0}\left(1+H_{R}\right.}\right)}{\mu_{T_{0}}}\right)
$$

$R_{b}>0, R_{c}>0$ and $R_{n}>0$ indicate that the initial viscosities of the solutions made by components B, C and NPs are greater than that of A respectively. Furthermore, $R_{\theta}>0$ in endothermic reactions while $R_{\theta}<0$ in exothermic ones.

\subsection{Numerical methods}

Similar to that discussed in the previous Chapters, the equations are first formulated in terms of vorticity $(\omega)$, stream function $(\psi)$, concentrations $\left(C_{j}, C_{n}\right)$ and temperature $(\theta)$ and then are solved with the Pseudo-Spectral method [77]. The base state equations for $C_{a}, C_{b}$ 
and $C_{n}$ are below while $\bar{C}_{c}, \bar{\theta}, \bar{V}$ is zero:

$$
\begin{gathered}
\frac{\partial \bar{C}_{a}}{\partial t}=\frac{\partial^{2} \bar{C}_{a}}{\partial x^{2}} \\
\frac{\partial \bar{C}_{b}}{\partial t}=\delta_{b} \frac{\partial^{2} \bar{C}_{b}}{\partial x^{2}} \\
\frac{\partial \bar{C}_{n}}{\partial t}=\delta_{n} \frac{\partial^{2} \bar{C}_{n}}{\partial x^{2}}-D a_{d e p} \bar{C}_{n}
\end{gathered}
$$

Adopting $\bar{C}_{a}=H(-x), \bar{C}_{b}=H(x)$ and $\bar{C}_{n}=C_{n 0}=H(-x)$ as the initial condition and zero flux boundary values, $\bar{C}_{a}(x, t)=\frac{1}{2} \operatorname{erf} c\left(\frac{x}{2 \sqrt{t}}\right), \bar{C}_{b}(x, t)=\frac{1}{2} \operatorname{erfc}\left(-\frac{x}{2 \sqrt{\delta_{b} t}}\right)$ and $\bar{C}_{n}(x, t)=$ $\frac{C_{n 0}}{2} \operatorname{erf} c\left(\frac{x}{2 \sqrt{\delta_{n} t}}\right)$. The perturbation equations are simply derived by subtracting the model equations from the base state equations:

$$
\begin{gathered}
\nabla^{2} \psi^{\prime}=-\omega^{\prime} \\
\omega^{\prime}=R_{b} N_{b}+R_{c} N_{c}+R_{n} N_{n}+R_{\theta} N_{\theta} \\
\frac{\partial C_{j}^{\prime}}{\partial t}=J_{j}+\delta_{j} \vec{\nabla} C_{j}^{\prime} \pm D a\left(\bar{C}_{a}+C_{a}^{\prime}\right)\left(\bar{C}_{b}+C_{b}^{\prime}\right)\left(\bar{C}_{n}+C_{n}^{\prime}\right) \\
\frac{\partial C_{n}^{\prime}}{\partial t}=J_{n}+\delta_{n} \vec{\nabla} \cdot\left(\left(1+H_{R} \theta^{\prime}\right) \vec{\nabla}\left(\bar{C}_{n}+C_{n}^{\prime}\right)\right) \\
+\delta_{T} \vec{\nabla} \cdot\left(\left(\bar{C}_{n}+C_{n}^{\prime}\right) \vec{\nabla} \operatorname{Ln}\left(1+H_{R} \theta^{\prime}\right)\right)-\delta_{n} \frac{\partial^{2} \bar{C}_{n}}{\partial x^{2}}-D a_{d e p} C_{n}^{\prime} \\
\frac{\partial \theta^{\prime}}{\partial t}=\lambda J_{\theta}+(1-\lambda) \frac{\partial \theta^{\prime}}{\partial x}+\operatorname{Le} \nabla^{2} \theta^{\prime}+\operatorname{sign}\left(H_{R}\right) D a\left(\bar{C}_{a}+C_{a}^{\prime}\right)\left(\bar{C}_{b}+C_{b}^{\prime}\right)\left(\bar{C}_{n}+C_{n}^{\prime}\right)
\end{gathered}
$$

Where

$$
\begin{gathered}
N_{i}=\frac{\partial \psi^{\prime}}{\partial x}\left(\frac{\partial \bar{X}_{i}}{\partial x}+\frac{\partial X_{i}^{\prime}}{\partial x}\right)+\left(1+\frac{\partial \psi^{\prime}}{\partial y}\right) \frac{\partial X_{i}{ }^{\prime}}{\partial y} \\
J_{i}=\frac{\partial \psi^{\prime}}{\partial x} \frac{\partial X_{i}^{\prime}}{\partial y}-\frac{\partial \psi^{\prime}}{\partial y}\left(\frac{\partial \bar{X}_{i}}{\partial x}+\frac{\partial X_{i}^{\prime}}{\partial x}\right)
\end{gathered}
$$

Where $X_{i}=\left(C_{j}, C_{n}, \theta\right)$. The perturbation BCs are now periodic in both longitudinal and transverse directions. Furthermore, $\psi^{\prime}, \omega^{\prime}, C_{c}^{\prime}, \theta^{\prime}$ are initially set zero, while the random 

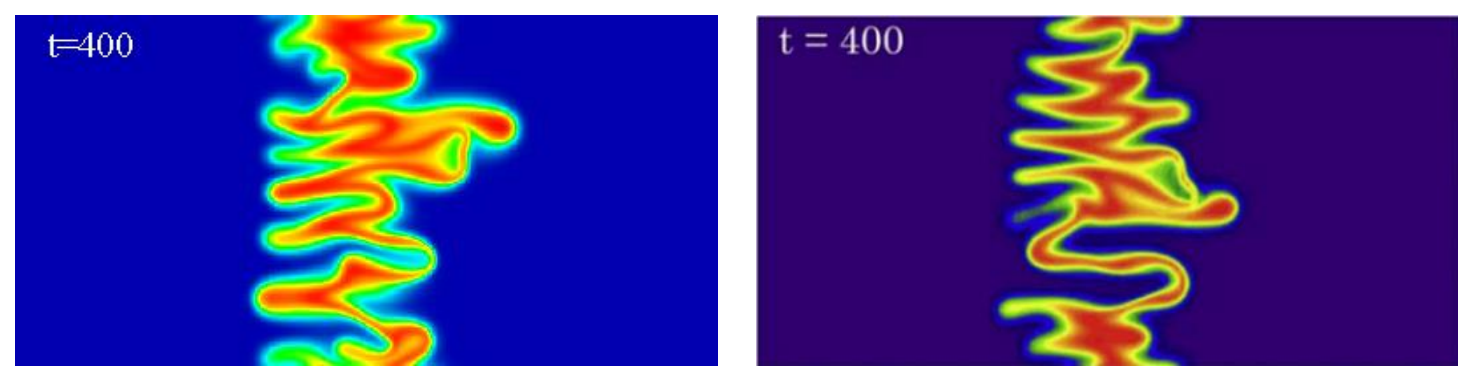

Figure 5.2: The contours of $C_{c}$ in the isothermal NP-free reactive system derived from the present study (left) and Hejazi and Azaiez [54] (right), $R_{b}=3, R_{c}=5, D a=0.5, P e=1024$, $A_{s}=2$.

number distributions are allocated for $C_{a}^{\prime}, C_{b}^{\prime}$ and $C_{n}^{\prime}$.

Finally, the numerical convergence has been checked and it was found that a grid of $256 \times 128$ is satisfactory for the range of considered parameters. The code has been validated for both binary and reactive systems in the absence of NPs. First, setting $R_{c}=R_{n}=R_{\theta}=$ $D a=0$ the results are compared with available results for binary systems. The results found to be in good agreement with each other. Subsequently, the finger configurations of a NP-free reactive system reported by [54] are compared with the results of the developed code by setting $R_{n}=R_{\theta}=0$ and $C_{n}=1$. As shown in Fig. 5.2 the fingers have similar structure qualitatively.

\subsection{Results and discussion}

The considered problem involves a large number of parameters. Therefore to narrow them, the reference values of $\lambda=1, L e=1, \delta_{j}=\delta_{n}=1, D a=1, D a_{\text {dep }}=0, A_{s}=2, P e=1024$ are adopted unless otherwise indicated. Furthermore, in order to investigate the direct coupled effect of the presence of NPs and the heat of reaction, $R_{\theta}$ is set to be zero. This choice will be in particular valid when the heat of the reaction is localized at the interface where the reaction takes place, resulting in large temperature gradients and in turn strong thermophoretic effects while the overall changes in the temperature are not strong enough to induce noticeable changes in the fluids viscosities. Note that in NP-free reactive systems 
the presence of the heat of reaction has no effects on the instabilities if $R_{\theta}=0$. Therefore, any changes in the dynamics of the system that may arise in the presence of NPs, will be interesting.

The analysis starts with NP-laden isothermal reactive systems. This analysis will allow development of a classification of the reactive systems in terms of the mobility ratios of the different chemical species. The analysis is then expanded to analyze the effects of the heat of reaction in each classified NP-laden systems for both exothermic and endothermic reactions. This will be conducted qualitatively through the contours of $C_{c}$ and quantitatively through the first moment of the transversely averaged product concentration as a representative of its center of mass and the cumulative concentration of the chemical products. The normalized first moment of the transversely averaged concentration of the products is defined as:

$$
x_{m}(t)=\frac{1}{P e} \int_{-\frac{P e}{2}}^{\frac{P e}{2}} x C_{c, a v}(x, t) d x
$$

Where

$$
C_{c, a v}=\frac{A_{s}}{P e} \int_{0}^{\frac{P e}{A_{s}}} C_{c}(x, y, t) d y
$$

Positive $x_{m}$ indicates that the products are mostly developed downstream and vice versa. The normalized cumulated value of the chemical product is further defined as

$$
\left(C_{c}\right)_{t}=\frac{A_{s}}{P e^{2}} \int_{-\frac{P e}{2}}^{\frac{P e}{2}} \int_{0}^{\frac{P e}{A_{s}}} C_{c}(x, y, t) d y d x
$$

It is expected that in the absence of NP deposition the value of $\left(C_{c}\right)_{t}$ increases monotonically as a result of the chemical reaction.

\subsubsection{Isothermal reactions}

In the absence of the heat of reaction $\left(H_{R}=0\right), \theta=0$ in the channel while the NP transport

equation reduces to the simple form of $\frac{\partial C_{n}}{\partial t}=\delta_{n} \frac{\partial^{2} C_{n}}{\partial x^{2}}-D a_{d e p} C_{n}$. This equation is coupled 
with the other governing equations through the rate of reaction and viscosity which distinguishes it from the NP-free reactive systems. In the NP-free reactive systems involving a $A+B \rightarrow C$ reaction with constant diffusivities, it was already reported that if the system is unstable before the reaction $\left(R_{b}>0\right)$, it remains unstable after the reaction regardless of the value of $R_{c}$. On the other hand in the case of an initially stable system $\left(R_{b} \leq 0\right)$, the subsequent instability condition after the occurrence of the reaction depends on $R_{c}$. Specifically, the viscosity distribution is monotonically decreasing if $0>\frac{R_{c}}{2}>R_{b}$ and the system is stable. On the other hand the system is unstable if $0<\frac{R_{c}}{2}<R_{b}$ where the viscosity is monotonically increasing or if $R_{c}\left(R_{b}-\frac{R_{c}}{2}\right)<0$ where the viscosity distribution is non-monotonic [53]. We will attempt here to extend these conclusions to NP-laden reactive $A+B+n \rightarrow C+n$ systems where the NPs have a catalytic role. Similarly, two systems are distinguished depending on whether the NP-laden systems are unstable or stable befor the reaction. As reported [106], in the absence of deposition which is the focus of this study, the NP-laden non-reactive system is unstable if $R_{b}-R_{n}>0$ and stable otherwise. Henceforth we will refer to these two non-reactive NP-laden systems as intrinsically unstable and intrinsically stable respectively. Adopting $R_{b}=3, R_{n}=2$ as an intrinsically unstable and $R_{b}=-0.5, R_{n}=2$ as the intrinsically stable systems, the variations of the one dimensional Log-viscosity with respect to the variable $\eta=\frac{x}{2 \sqrt{t}}$ are presented at an asymptotic large time at Fig. 5.3. Insets of contours of $C_{c}$ obtained from non-linear simulations are included to illustrate the instability condition and show the finger configurations. The figure shows that, consistent with the results of the NP-free reactive systems, in the NP-laden reactive systems, intrinsically unstable systems remain unstable after the reaction as it generates either monotonically increasing or non-monotonic viscosity distributions. On the other hand, initially stable systems may become unstable as a result of the non-monotonicity in the viscosity distribution after the reaction. Furthermore, similar to the NP-free systems, in both cases the system with $R_{c}=R_{b}$ obeys the one-dimensional viscosity distribution in the form of error-function. This is the less unstable case after the reaction in the intrinsically unstable 


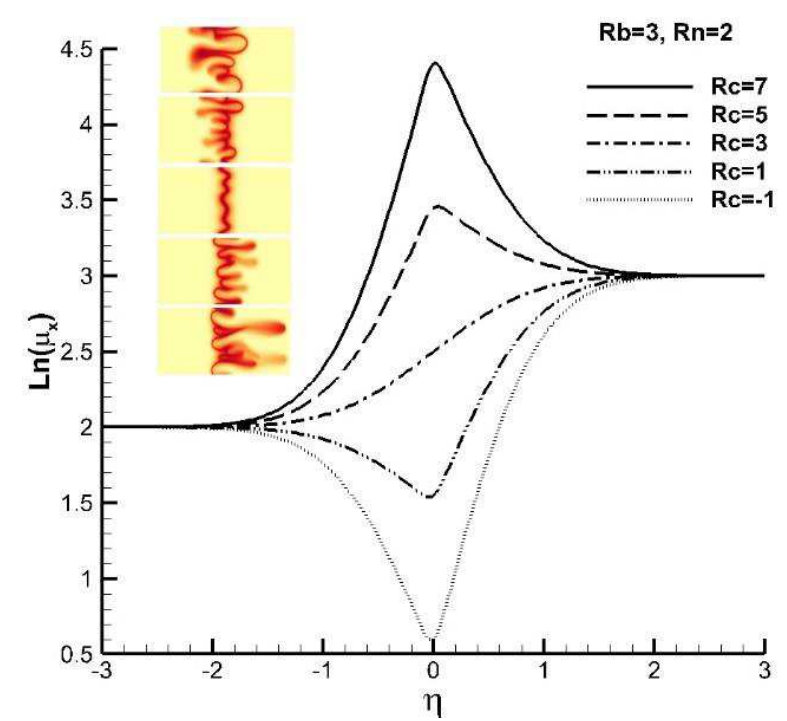

(a) Intrinsically unstable

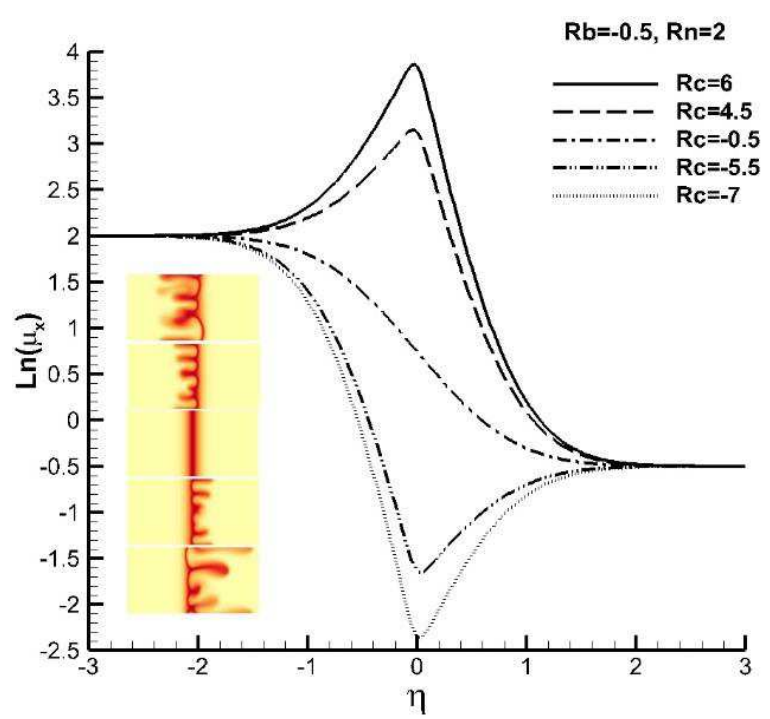

(b) Intrinsically stable

Figure 5.3: One dimensional $\log$-viscosity variation with respect to $\eta=\frac{x}{2 \sqrt{t}}$ at asymptotically large times $(t \rightarrow \infty)$ along with insets of corresponding contours of $C_{c}$ derived from NLS. The contours are represented at $t=1000$ for intrinsically unstable and $t=1300$ for intrinsically stable systems.

system according to the contours of $C_{c}$. However as $\left|R_{c}-R_{b}\right|$ increases, both intrinsically stable and unstable systems are prone to more unstable situation as a result of the chemical reaction.

From Fig. 5.3 one may note that the viscosity variation may be different for $\eta>0$ or $\eta<0$ henceforth referred to as the leading zone and trailing zone respectively [53]. Comparing the viscosity variations and the finger configurations, it is clear that monotonically increasing viscosities in any zone lead to more finger development in that region. Unlike the NP-free systems, the present viscosity variation is affected by $R_{n}$ in addition to $R_{b}$ and $R_{c}$ in identical diffusivities.

To determine the exact condition for instability in each zone, the one dimensional form of the governing equations in the direction of the flow is adopted to obtain the viscosity distribution $\mu_{x}$. Accordingly, assuming $\delta_{j}=1$, one concludes that $\frac{\partial\left(C_{a x}+C_{b x}+2 C_{c x}\right)}{\partial t}=\frac{\partial^{2}\left(C_{a x}+C_{b x}+2 C_{c x}\right)}{\partial x^{2}}$ and so with the defined boundary and initial conditions, $C_{a x}+C_{b x}+2 C_{c x}=1$. Furthermore, it can be shown that $C_{b x}-C_{a x}=\operatorname{erf}\left(\frac{x}{2 \sqrt{t}}\right)$. Taking advantage of these results and further 
assuming $\delta_{n}=1$ to get rid of double diffusivity effects and simply denoting $C_{n 0} R_{n}$ by $R_{n}$, Eq. 5.28 is obtained in the absence of deposition. Note that in the presence of deposition, NPs are gradually removed from the system and the effect of $R_{n}$ decreases with the passage of time. Furthermore, not only does the rate of deposition diminish the effect of $R_{n}$, but it also has a direct effect on the chemical product concentration. So, the coupled effect of $D a_{d e p}$ on the viscosity of the system and the production rate may have different effects on the dynamics of the flow. This however is not the focus of the present study and may be the subject of a separate investigation.

$$
\frac{2}{\mu_{x}} \frac{\partial \mu_{x}}{\partial x}=\left(R_{c}-R_{n}\right)\left(-\frac{C_{a x}}{\partial x}\right)+\left(2 R_{b}-R_{c}-R_{n}\right) \frac{C_{b x}}{\partial x}
$$

If $\frac{\partial \mu_{x}}{\partial x}>0$ the viscosity distribution is monotonically increasing and the system is unstable and vice versa. Since $\frac{\partial C_{b x}}{\partial x}$ and $\left(-\frac{C_{a x}}{\partial x}\right)$ are positive, one can conclude that for $R_{n} \geq 0, R_{b}$ has destabilizing and $R_{n}$ has stabilizing effects, while the effect of $R_{c}$ on the instability is non-monotonic. As expected, this equation allows to distinguish two zones that develop as a result of the reaction; the leading zone with $R_{L}=2 R_{b}-R_{c}-R_{n}$ and the trailing one with $R_{T}=R_{c}-R_{n}$. Therefore, $R_{L}>0$ and $R_{T}>0$ indicate unstable zones and vice versa. It is concluded that the viscosity distribution will be monotonically increasing if $R_{L}>0$ and $R_{T}>0$ while it is monotonically decreasing if $R_{L}<0$ and $R_{T}<0$. However, if $R_{L} R_{T}<0$ the viscosity distribution may be non-monotonic where the flow is unstable. The special case of $R_{b}=R_{c}$ reduces the problem to that of a non-reactive NP-laden displacement while $R_{c}=R_{n}$ represents a neutrally stable trailing zone which implies no extremum in the viscosity distribution in this zone. However, the system with $R_{b}>R_{n}=R_{c}$ is more unstable than the intrinsically unstable system as the viscosity gradient now is confined in the smaller leading zone than the total channel. On the other hand with $R_{b}<R_{n}=R_{c}$ the system is stable.

Assuming $R_{b}>R_{n}$ that represents an intrinsically unstable system, one can conclude 
Table 5.1: Characteristics of classified isothermal systems.

\begin{tabular}{|c|c|c|}
\hline System & Before the reaction & Unstable zone after the reaction \\
\hline Unstable & Unstable $\left(R_{b}>R_{n}\right)$ & Both $\left(2 R_{b}-R_{c}-R_{n}>0, R_{c}>R_{n}\right)$ \\
$I U D$ & Unstable $\left(R_{b}>R_{n}\right)$ & Leading $\left(2 R_{b}-R_{c}-R_{n}>0, R_{c}<R_{n}\right)$ \\
$I U U$ & Unstable $\left(R_{b}>R_{n}\right)$ & Trailing $\left(2 R_{b}-R_{c}-R_{n} \leq 0, R_{c}>R_{n}\right)$ \\
Stable & Stable $\left(R_{b} \leq R_{n}\right)$ & None $\left(2 R_{b}-R_{c}-R_{n} \leq 0, R_{c}<R_{n}\right)$ \\
$I S D$ & Stable $\left(R_{b} \leq R_{n}\right)$ & Leading $\left(2 R_{b}-R_{c}-R_{n}>0, R_{c}<R_{n}\right)$ \\
$I S U$ & Stable $\left(R_{b} \leq R_{n}\right)$ & Trailing $\left(2 R_{b}-R_{c}-R_{n} \leq 0, R_{c}>R_{n}\right)$ \\
\hline
\end{tabular}

that $2 R_{b}-R_{c}-R_{n}>-\left(R_{c}-R_{n}\right)$. If $2 R_{b}-R_{c}-R_{n}>0$, then $R_{c}-R_{n}$ can be positive or negative while if $2 R_{b}-R_{c}-R_{n} \leq 0$, then $R_{c}-R_{n}$ can only be positive. Accordingly, as long as $R_{b}>R_{n}$ at least one of the fronts is unstable, indicating that the intrinsically unstable system remains unstable after the reaction regardless of the value of $R_{c}$. Similarly, in the case $R_{b} \leq R_{n}$ corresponding to an intrinsically stable system, one has $2 R_{b}-R_{c}-R_{n} \leq-\left(R_{c}-R_{n}\right)$. If $2 R_{b}-R_{c}-R_{n}>0$ then $R_{c}-R_{n}$ can only be negative while if $2 R_{b}-R_{c}-R_{n} \leq 0, R_{c}-R_{n}$ can be either positive or negative. As a result, depending on the value of $R_{c}$, the intrinsically stable system will either remain stable or become unstable as a result of the reaction. These logical expressions further imply that depending on the particular values of $R_{b}, R_{c}$ and $R_{n}$ where $R_{c} \neq R_{b}$ and $R_{n}$ six different classes of systems can be identified in terms of their finger configuration. We will refer to them as Unstable (intrinsically unstable, both zones unstable after the reaction), IUD (intrinsically unstable, the leading zone unstable after the reaction where the products are mostly developed downstream), IUU (intrinsically unstable, the trailing zone unstable after the reaction where the products are mostly developed upstream), Stable (intrinsically stable, both zones stable after the reaction), ISD (intrinsically stable, the leading zone unstable after the reaction where the products are mostly developed downstream) and $I S U$ (intrinsically stable, the trailing zone unstable after the reaction where the products are mostly developed upstream). Table 1 summarize this classification with the representative mobility ratios for each case. The qualitative viscosity variation along the channel can be found in Fig. 5.3 for all the cases discussed.

Fig. 5.4 presents the variation of $x_{m}$ with time for two values of $D a$ and shows where the 


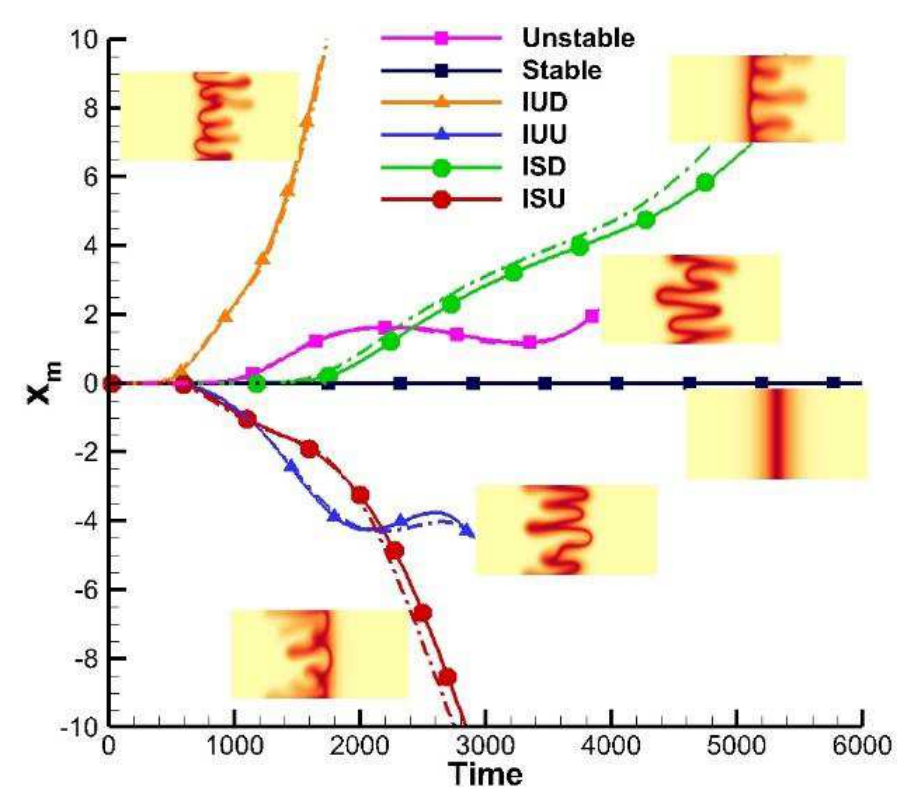

Figure 5.4: Variation of the normalized first moment of the transversely averaged product concentration with the inset of contours of $C_{c}$. Dashed lines represent those systems with $D a=10$.

products in each classified systems are developed. The representative systems for each class are $R_{b}=3, R_{n}=2, R_{c}=2.5$ (Unstable), $R_{b}=3, R_{n}=5, R_{c}=4$ (Stable), $R_{b}=3, R_{n}=$ $2, R_{c}=1(I U D), R_{b}=3, R_{n}=2, R_{c}=4(I U U), R_{b}=-0.5, R_{n}=2, R_{c}=-5(I S D)$, $R_{b}=-0.5, R_{n}=2, R_{c}=5(I S U)$. Note that $x_{m}=0$ in the Stable system as the system is diffusion dominated. On the other hand, as predicted, the products are more developed downstream $\left(x_{m}>0\right)$ in the $I U D$ and $I S D$ systems while they are more developed upstream $\left(x_{m}<0\right)$ in the $I U U$ and $I S U$ cases. Furthermore, $x_{m}>0$ in the Unstable case as $R_{L}>R_{T}$. One may notice that the increase in $x_{m}$ is monotonic in both $I U D$ and $I S D$ systems as the direction of bulk flow is aligned with the fact that $R_{L}>0$. In the $I S U$ system however, $x_{m}$ is decreasing monotonically as the products experience strong resistance to flow downstream and so reverse to flow upstream instead. On the other hand the behavior of $x_{m}$ in the $I U U$ and Unstable systems is non-monotonic, indicating a competition between the bulk flow and the viscosity contrast effects.

NPs have a catalytic role in the present study and accordingly their transport has an impact on the total amount of chemical product. To analyze the effects of NPs on production 
and compare the trends with those of NP-free reactive systems, the variation in time of the total accumulated production is plotted in Fig. 5.5 for both NP-laden intrinsically unstable and stable systems by varying $R_{c}$. Again $R_{b}=3, R_{n}=2$ and $R_{b}=-0.5, R_{n}=2$ are selected as the mobility ratios of the NP-laden intrinsically unstable and stable systems respectively. Note that in these figures as $R_{c}$ is increased, the type of systems changes from $I U D$ to Unstable and finally to $I U U$ in the intrinsically unstable case, and from $I S D$ to Stable and finally to $I S U$ in the intrinsically stable systems. Fig. 5.5 shows that in the Log-Log scale $\left(C_{c}\right)_{t}$ increases linearly in the diffusive regime similar to the NP-free reactive systems. Furthermore, in the $I U D$ system increasing $R_{c}$ leads to smaller chemical production until the system switches to an Unstable type $\left(R_{c}=2.5\right)$. The Unstable system has the lowest amount of the chemical product. Further increase in $R_{c}$ changes the type of the system to $I U U$ in which the amount of products starts to increase. Similarly, according to Fig. 5.5b, as $R_{c}$ increases the amount of chemical product in the $I S D$ type decreases until the system becomes Stable $\left(R_{c}=-0.5\right)$ where the amount of chemical product attains a minimum. Further increase of $R_{c}$ changes the system to $I S U$ type where the amount of chemical product is increased. These trends were observed with other choices of the values of the viscosity ratios and with a higher reaction rate of $D a=10$. In the next section the present results will be extended to see the effect of the heat of reaction in the NP-laden catalytic systems.

\subsubsection{Non-isothermal reactions}

It is known that NP free thermo-viscous fingering instabilities both in the presence and absence of reaction develops as a result of the change in viscosity through $R_{\theta}[6,42,48]$. Accordingly, if $R_{\theta}=0$, heat transfer does not affect the instability in the case of NP-free systems. However, since the temperature gradient resulting from the heat of reaction affects the transport of NPs, one may suspect that this effect may change the dynamics and the chemical products even if $R_{\theta}=0$. To analyze this effect the results in the previous section 


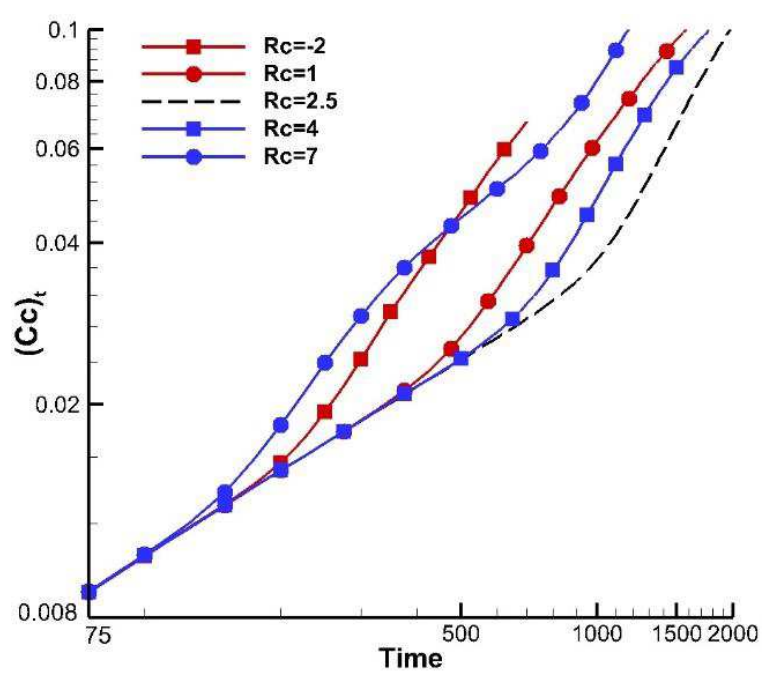

(a) Intrinsically unstable

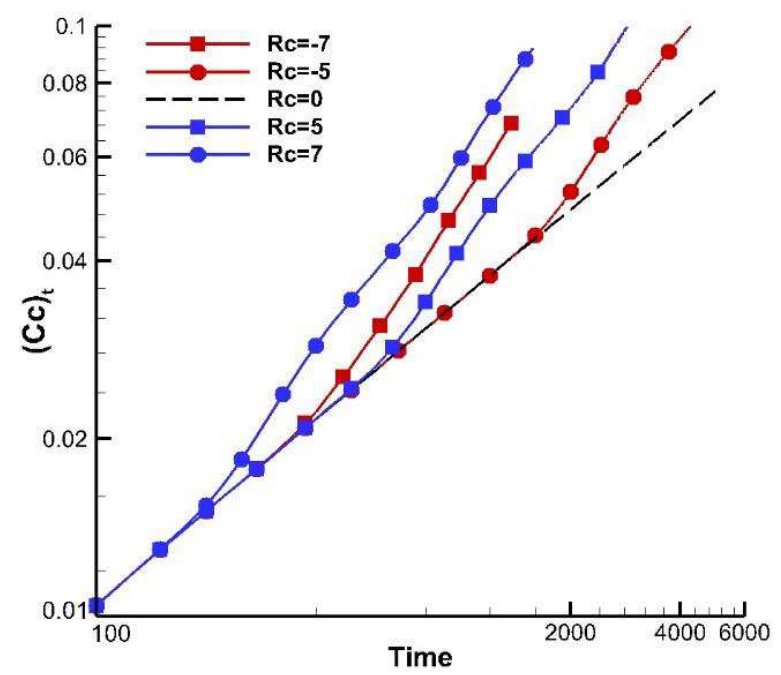

(b) Intrinsically stable

Figure 5.5: The effect of $R_{c}$ on the total amount of chemical products in the NP-laden isothermal reactive systems in the $\log$-Log scale.

are extended to include the heat of reaction but still for $R_{\theta}=0$. Specifically, the effect of temperature driven NP transport which is represented by thermophoretic diffusivity $\delta_{T}$ is investigated in the already introduced representative Unstable, IUD, IUU, Stable, ISD and $I S U$ systems while $H_{R} \neq 0$ for both exothermic and endothermic reactions.

\section{Exothermic reactions}

The objective here is to investigate the coupled effect of $H_{R}$ and $\delta_{T}$ while the other heat transfer related properties are fixed. The analysis is first conducted for an exothermic reaction where the reference value of $H_{R}=0.1$ is adopted. Fig. 5.6 shows the variation of $x_{m}$ for different thermophoretic diffusivities with insets of contours of the chemical product concentration $C_{c}$. The solid curves represent the reference systems at $\delta_{T}=0$ and the dotted ones correspond to higher thermophoretic diffusivities. It is clear that for the set of adopted parameters, the variation of $x_{m}$ in the absence of thermophoretic diffusivity is virtually identical to that in the isothermal system. However, at larger thermophoretic diffusivities the change in the variation of $x_{m}$ is considerable. The figure shows that as $\delta_{T}$ increases, the transition time from the diffusion to the convective regime is reduced for systems with $x_{m} \geq 0$ 
and vice versa. Accordingly, at earlier times in convective regime thermophoresis leads to a systematic tendency for $x_{m}$ to shift to more absolute values in systems with $x_{m} \geq 0$ and to less absolute values in systems with $x_{m}<0$.

At later time, $\left|x_{m}\right|$ still systematically increases with $\delta_{T}$ in $I U D$ (except for a short interval) and $I S D$ where only the leading zone is unstable. According to the finger configurations, this effect is attributed to more developed forward fingers or most importantly to the suppression of reverse fingering at higher $\delta_{T}$. The same increasing trend is observed in the Stable case at high enough values of $\delta_{T}$ where the system becomes unstable with fingers developing downstream. Similar trends have been obtained in systems with larger Damköhler number, $D a=10$.

In the $I U U$ and $I S U$ systems where only the trailing zone is unstable, a non-monotonic trend is observed in the variation of $x_{m}$. However, at higher $\delta_{T}$ where thermophoretic effects are stronger, a general decreasing trend in the absolute value of $x_{m}$ with increasing $\delta_{T}$ can be reported. According to finger configurations in both systems, backward fingers are suppressed at higher values of $\delta_{T}$ which is the main reason for this trend. Further examination of the finger configurations reveals that for $I U U$ and $I S U$ systems, the products become confined in a narrow region as $\delta_{T}$ increases. In these systems the leading zone is locally stable and acts as a barrier to the upcoming flow. The upcoming flow as a result, returns and feeds the reverse fingers as the trailing front is unfavorable. However, with the suppression of reverse fingering at higher $\delta_{T}$, the products have less possibility to move backward as well, and so get confined afterwards. The suppression of the forward fingers in addition to the backward fingers in $I U U$ system is also attributed to this feature. Note that the backward finger suppression only delays the transport of the products upstream and the absolute value of $x_{m}$ still increases at later times. Further analysis shows that this trend is virtually similar in higher reaction rates.

Finally, in the Unstable case the suppressed backward fingers now find the way to develop downstream as the leading zone is unstable. However, the forward fingers cannot accommo- 


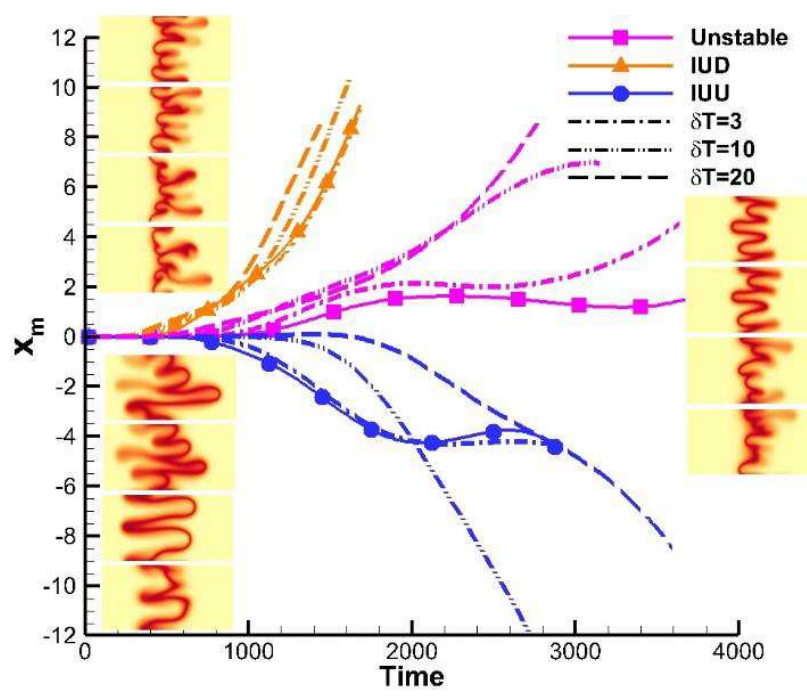

(a) Intrinsically unstable

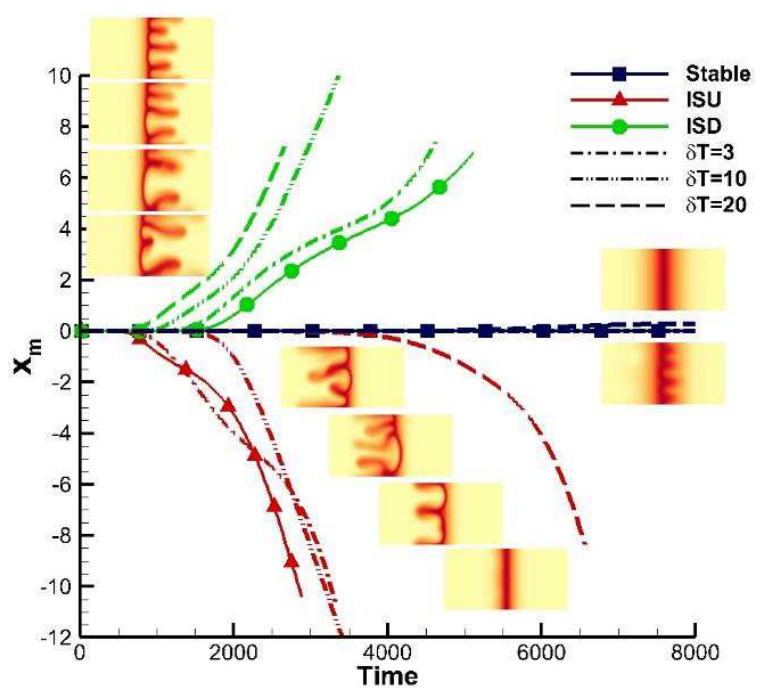

(b) Intrinsically stable

Figure 5.6: The variation of $x_{m}$ with time at different thermophoretic diffusivities in both intrinsically unstable and stable reference systems along with the inset of the contours of $C_{c}$. The contours are depicted at identical times in each systems. The solid lines represent the systems with $\delta_{T}=0$.

date all the upcoming flow. Accordingly, one may observe the confined products with few stretched fingers downstream at high $\delta_{T}$. So, the general increasing trend for $x_{m}$ with $\delta_{T}$ is observed and this trend is most noticeable at late times.

In summary, it is observed that the thermophoretic effects resulting from the heat of reaction alter both the finger configurations and the transport of the products. It can cause more developed or complex finger configurations in systems with unstable leading zone, leads to the suppression of backward product fingers, make the stable system unstable, and confine the chemical products in systems with leading stable zones (or even weak unstable zone). This affects the center of mass of the products as discussed above.

Fig. 5.7 shows the corresponding one dimensional viscosity distribution of the reference systems in the presence and absence of thermophoresis. Analyzing this figure elucidates the underlying reasons behind the changes in the variation of $x_{m}$ shown in Fig. 5.6. The coupled effects of the viscosity increase at $x<0$ and its decrease at the center cause the generation or intensification of locally stable regions in the trailing zone at $\delta=20$ and result in a stronger 


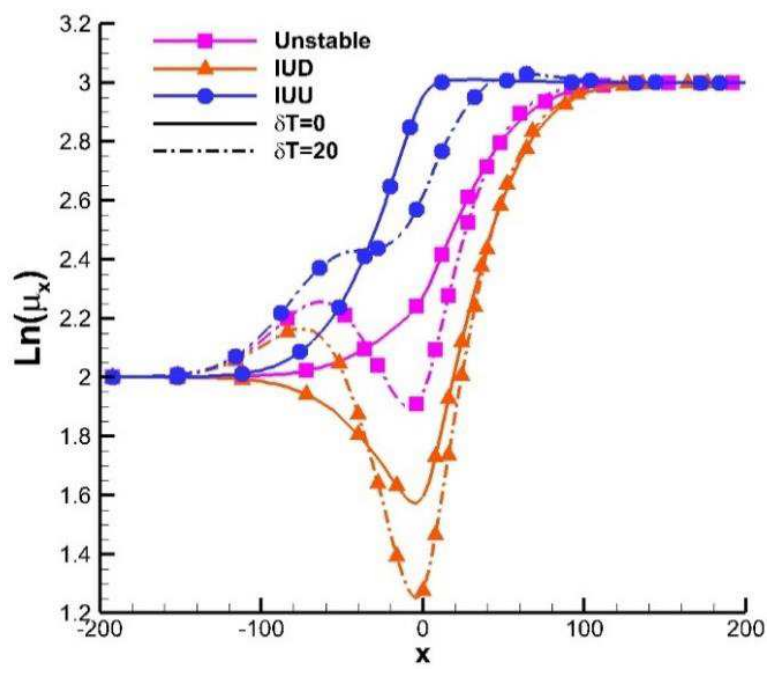

(a) Intrinsically unstable

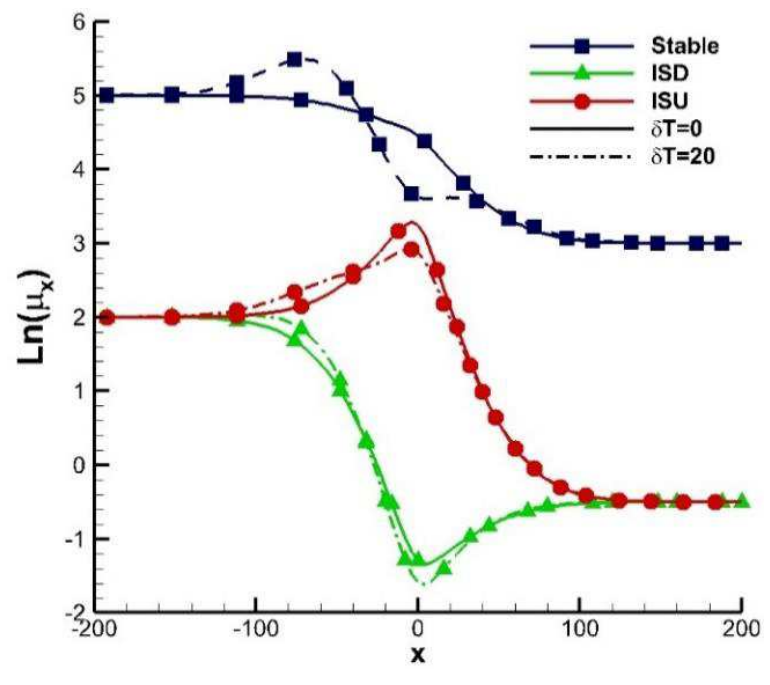

(b) Intrinsically stable

Figure 5.7: The one dimensional viscosity variation in the reference systems in the presence $\left(\delta_{T}=20\right)$ and the absence of thermophoresis.

resistance to the transport of the products upstream (in all systems except Stable). This important feature along with a sharper increase of the viscosity in systems with unfavorable leading zone (Unstable, IUD, ISD) at $x>0$ and the fact that the monotonically decreasing viscosity distribution in the Stable system becomes non-monotonic at $\delta_{T}=20$, lead to the reported trends in the variations of $x_{m}$ and the finger configuration.

One may wonder what triggers these changes in the flow behavior when the viscosities of the fluids are not changing with temperature variations $\left(R_{\theta}=0\right)$ and what is the role of heat of reaction? To attempt to answer this question, we examine the one dimensional NP transport equation, Eq. 5.10:

$$
\frac{\partial C_{n x}}{\partial t}=\delta_{n}\left(1+H_{R} \theta_{x}\right) \frac{\partial^{2} C_{n x}}{\partial x^{2}}+H_{R}\left(\delta_{n}+\frac{\delta_{T}}{1+H_{R} \theta_{x}}\right) \frac{\partial \theta_{x}}{\partial x} \frac{\partial C_{n x}}{\partial x}+\delta_{T} \frac{\partial^{2} \operatorname{Ln}\left(1+H_{R} \theta_{x}\right)}{\partial x^{2}} C_{n x}
$$

The first and second terms on the RHS represent diffusion-like and convection-like contributions respectively while the third one is a source/sink-like term. This equation is solved along with the other coupled one-dimensional heat and concentration equations for different 
values of $\delta_{T}$ and $H_{R}$ with the already-defined reference parameters. Note that since $u=0$ in these 1D equations, the viscosity is decoupled from the equations and the choice of mobility ratios does not have any effects on the concentrations and temperature distributions. Accordingly with any arbitrary choice of mobility ratios, Fig. 5.8 shows the NP concentration distribution along the channel for different values of $\delta_{T}$ and $H_{R}$ including the corresponding scaled variation of the convective term: Conv $=H_{R}\left(\delta_{n}+\frac{\delta_{T}}{1+H_{R} \theta_{x}}\right) \frac{\partial \theta_{x}}{\partial x}$ and the source/sink term: $S r c=\delta_{T} \frac{\partial^{2} \operatorname{Ln}\left(1+H_{R} \theta_{x}\right)}{\partial x^{2}}$. Note that Conv $=S r c=0$ at both ends of the domain and clearly everywhere in the case of the isothermal system. Conv $\leq 0$ with a local minimum for $x>0$ while Conv $\geq 0$ with a local maximum for $x<0$. Positive Conv leads to a negative thermophoretic velocity (NP velocity as a result of temperature gradient $V_{t} \propto-\frac{\partial \theta_{x}}{\partial x}$ ) and vice versa. On the other hand $S r c$ experiences a local minimum with a negative value at the center and two positive local maxima at each side.

The figure shows that by increasing $\delta_{T}$ and $H_{R}$, there is more accumulation of NPs at $x<0$ and depletion at the center. Following a previous flow analysis [105], this behavior is a direct consequence of the temperature gradient driven convective transport of the NPs from the center to either sides of the channel. More specifically, the convected NPs from the center increase the local concentration of NPs at either side while NP concentration is decreased at the center, in the position of positive and negative Src respectively. From the definition, both Conv and Src increase with increasing $\delta_{T}$ and $H_{R}$. This indicates that the two thermal-related terms, $\delta_{T}$ and $H_{R}$ have synergic effects in the accumulation of NPs at either side of the channel and their depletion at the center. Accordingly as $R_{n}>0$, a viscosity decrease at the center and its increases at $x<0$ (considerably) and $x>0$ (less pronounced) is expected if $\delta_{T}$ and $H_{R}$ increase. This will affect the transport of the products, the variation of $x_{m}$ and the total chemical production. Note that there is not any accumulation or depletion if $\delta_{T}=0$ although there is a convective velocity driven by the temperature gradient and Brownian diffusivity. This is because the convected NPs then diffuses according to the diffusive term $\delta_{n}\left(1+H_{R} \theta_{x}\right) \frac{\partial^{2} C_{n x}}{\partial x^{2}}$ in the absence of Src. 


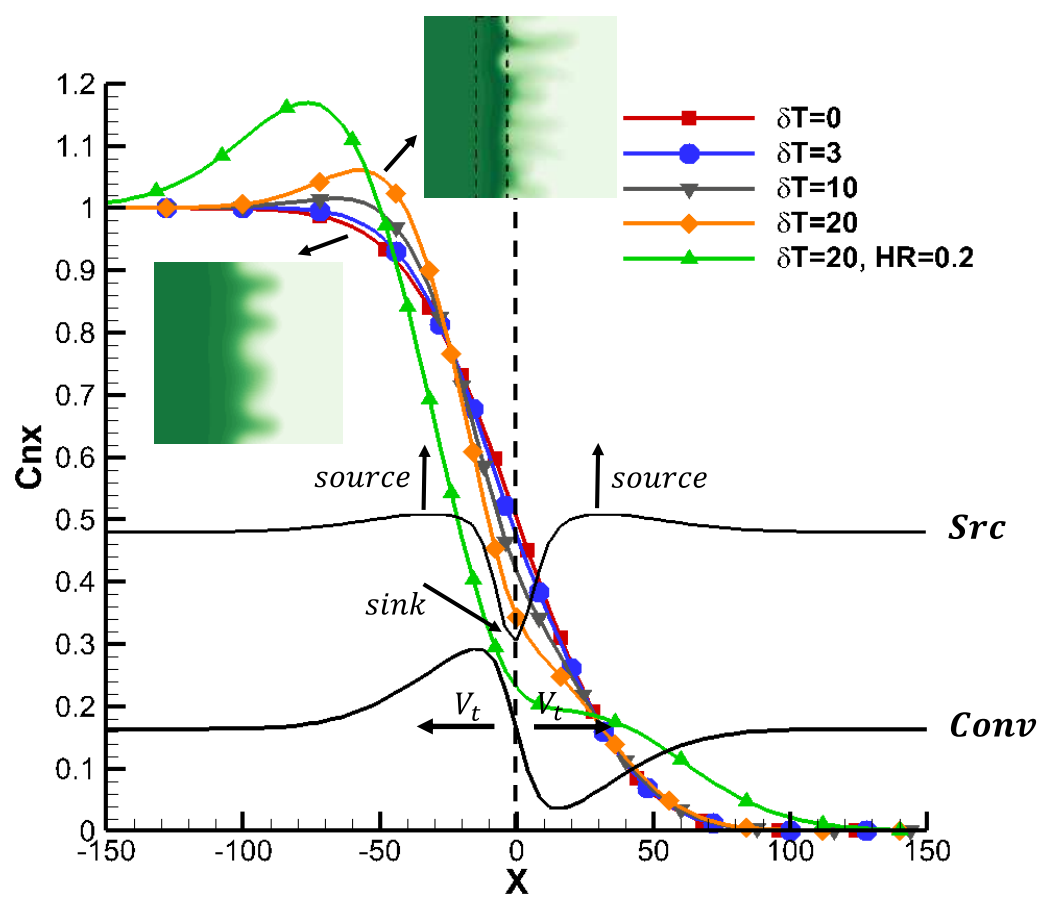

Figure 5.8: The one dimensional NP concentration variation at different $\delta_{T}$ with constant $H_{R}=0.1$, and at two $H_{R}$ with constant $\delta_{T}=20$ including the scaled convective and source/sink terms variation. The representative inset of the contours of $C_{n}$ derived from NLS are attached for $\delta_{T}=0$ and $\delta_{T}=20$.

Now with the change in the transport of NPs discussed above, the question is how the amount of chemical products is changing in NP-laden reactive systems. We will examine next how the accumulated amount of chemical products is changing in the presence of thermophoretic effects with respect to the case in the absence of thermophoresis. Note that since with the set of parameters used, the total chemical production in the isothermal system is almost identical with that for $\delta_{T}=0$, one can extend these results to compare it with the isothermal case as well.

Defining $\left(C_{c}\right) r_{i}=\frac{\left(C_{c}\right)_{t}\left(\delta_{T}=i\right)-\left(C_{c}\right)_{t}\left(\delta_{T}=0\right)}{\left(C_{c}\right)_{t}\left(\delta_{T}=0\right)}$, Fig. 5.9 shows the variation of $\left(C_{c}\right) r_{i}$ over time in the representative intrinsically unstable and stable systems respectively. $\left(C_{c}\right) r_{i}>0$ indicates that chemical production is larger than the case in the absence of thermophoresis and vice versa. According to Fig. 8a, the presence of thermophoresis leads in a first stage of the flow to a larger production in both the $I U D$ and Unstable systems. This trend is however subsequently reversed and $\left(C_{c}\right) r_{i}$ starts actually to decrease becoming later negative, imply- 
ing that thermophoresis ultimately leads to smaller chemical production. Further analysis which for brevity is not shown here indicates that depending on the choice of parameters, the final value of $\left(C_{c}\right) r_{i}$ may not always be negative in these systems. Opposite trends are found in the IUU system where the presence of thermophoresis results first in a decrease and then an increase of the relative chemical production. According to this figure, depending on the choice of parameters, the final relative production value in this case can actually lead to positive $\left(C_{c}\right) r_{i}$ indicating ultimate stronger chemical production than in the absence of thermophoretic effects. Note also that with increasing $\delta_{T}$, chemical production is intensified in all the cases.

In contrast to the intrinsically unstable case, thermophoresis always leads to a positive values of $\left(C_{c}\right) r_{i}$ in the $I S D$ and negative ones in the $I S U$ systems (except for a short interval when $\delta_{T}=3$ ). This indicates that thermophoresis increases the chemical production in the $I S D$ and decreases it in the $I S U$ systems and this trend is intensified by increasing $\delta_{T}$. Finally, interestingly large enough values of $\delta_{T}$ can lead to larger chemical production even

in the representative Stable system as a direct effect of the developed instabilities. The analysis shows virtually identical trends with higher reaction rate of $D a=10$ in all systems which for brevity are not shown here.

\section{Endothermic reactions}

In this section the previous analysis is extended to endothermic reactions. To start the analysis, let's examine the energy equation, Eq. 5.11. In this equation $\theta>0$ and $\operatorname{sgn}\left(H_{R}\right)=+1$ in the exothermic reaction while $\operatorname{sgn}\left(H_{R}\right)=-1$ and $\theta<0$ in the endothermic one. Accordingly, it is clear that with all parameters fixed, $\theta_{\text {endo }}(x, y)=-\theta_{\text {exo }}(x, y)$ if the distribution of $C_{a}, C_{b}$ and $C_{n}$ are not changed by changing the type of reaction. In particular by substituting $\theta(x, y) \rightarrow-\theta(x, y)$, the transport equation of the NPs and other components do not change if $H_{R} \rightarrow-H_{R}$. In addition, the viscosity distribution and the Darcy's law will not change as long as $R_{\theta} \rightarrow-R_{\theta}$ or $R_{\theta}=0$. So, one can extend all the conclusion discussed 


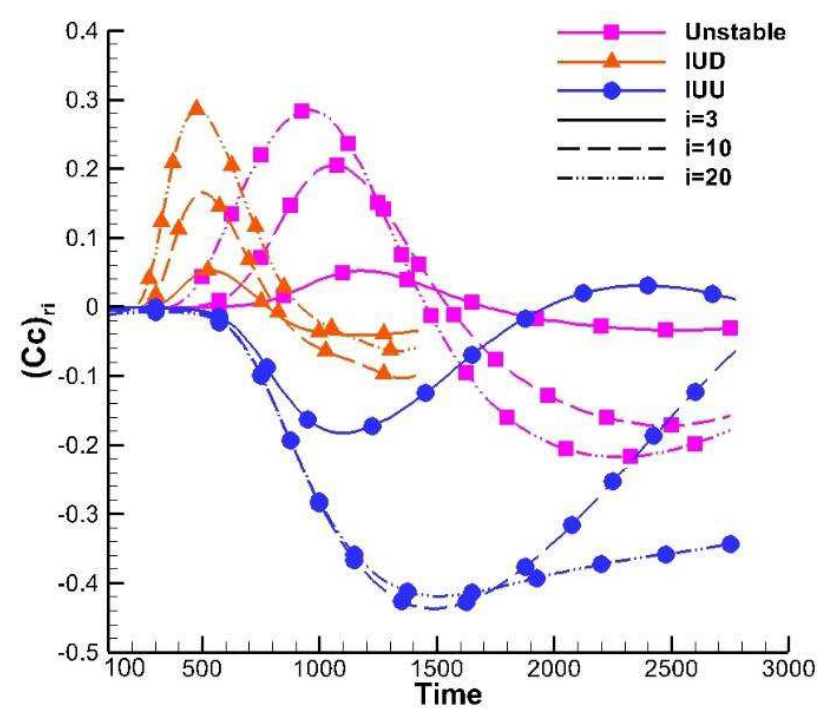

(a) Intrinsically unstable

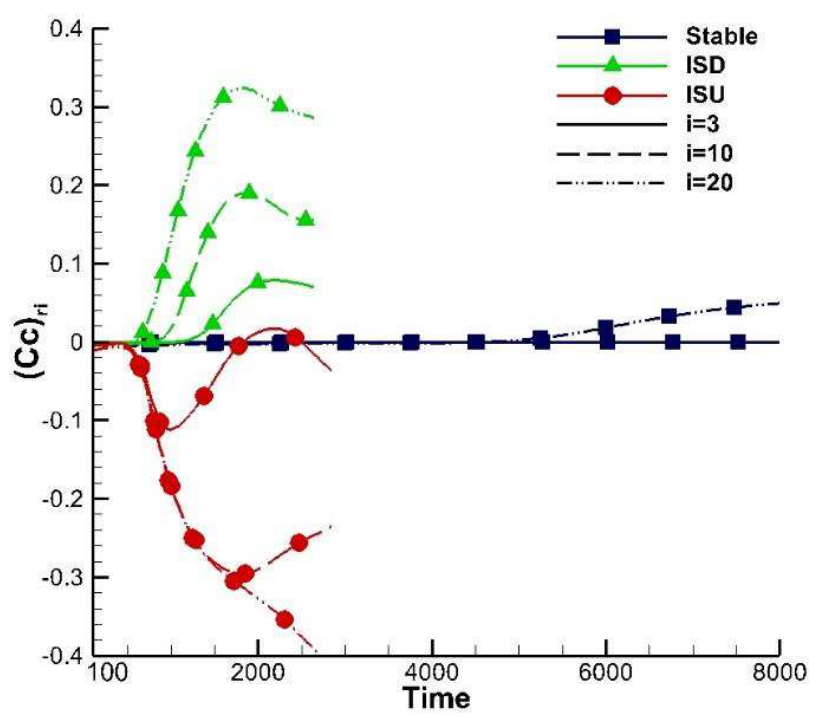

(b) Intrinsically stable

Figure 5.9: Variation of $\left(C_{c}\right)_{r_{i}}$ in the representative systems at different thermophoretic diffusivities

about the exothermic reaction to the endothermic one if $H_{R} \rightarrow-H_{R}$. This conclusion was validated by NLS. 


\section{Chapter 6}

\section{Conclusion}

The flow instability in homogeneous porous media is investigated in the presence of nanoparticles (NPs) dispersed in the displacing fluid. The study is conducted in three phases. In the first phase, the problem is analyzed under isothermal conditions. Here, the additional NP related properties namely the viscosity of the nanofluid, the Brownian diffusion and the NP deposition rate are introduced and their effects on the stability and the dynamics of the flow are discussed. Results show that for unit diffusivities and in the absence of deposition, the instability condition is governed by the parameter $R=R_{b}-R_{n}-R_{a} . R_{a}, R_{b}$ and $R_{n}$ are the Log-viscosity ratios of the invading fluid A, displaced fluid B and the nanofluid respectively to that of the base fluid. Specifically, if $R>0$ the system is unstable while it is stable otherwise. This implies that the presence of NPs in the displacing fluid can both modify the instability of an initially unstable system or make it totally stable. The study is then extended to include the effects of the deposition rate. It was found that the effects of NPs in this condition are limited only to the control of the instabilities and making an initially unstable binary system less unstable. As long as $R_{b}>R_{a}$, the instabilities grow over time even if $R_{a}+R_{n}>R_{b}$. Moreover, the LSA shows that NPs do not have any effect on the long wave instability of the system and hence the instability condition is identical to that for the binary system. In other words, the system is unstable when $R_{b}>R_{a}$ or $R_{b}<R_{a}$ 
and $\delta_{b}<\frac{R_{b}}{R_{a}}$ or $R_{b}=R_{a}>0\left(R_{a}<0\right)$ and $\delta_{b}<1\left(\delta_{b}>1\right)$. This highlights the critical role of the diffusion rate of the fluids with respect to that of the NPs. Further analysis shows that increasing $\delta_{n}$ makes the flow more unstable but it cannot however make a stable binary system unstable. The more unstable situation also appears when $\delta_{b}<1$ in which case the fingers can easily be stretched and split as a result of the stronger cross flow. The opposite scenario takes place for $\delta_{b}>1$. Finally, it is found that as $D a_{d e p}$ increases, the initially unstable flow becomes more unstable. It was discussed that this is due to the fact that NPs are constantly eliminated from the system and as a result modify the viscosity distribution towards a more unstable situation.

The study is then extended to include the thermal effects in the second phase. This phase of the study introduced an additional transport mechanism of NPs called thermophoresis and then analyzed its effect in connection with other properties, especially the Brownian diffusion. The analysis is conducted for two representative systems: $H D C$, where a Hot fluid Displaces a Cold fluid and $C D H$, where a Cold fluid Displaces a Hot fluid. In the HDC system, the NP concentration and temperature gradients are in the same direction. As a result, Brownian diffusion and thermophoresis act synergically to transport NP downstream towards the cold fluid in the low resistance paths created by the forward-growing fingers. It is found that increasing $\delta_{T}$ makes an initially unstable NP-laden system more unstable, which is similar to the effects of $\delta_{n}$. The destabilizing effects of thermophoresis in the HDC flows are extended to initially stable and intrinsically stable systems. On the other hand, the gradient of NP concentration and temperature in the CDH system are in opposite directions. As a result, Brownian diffusion still tends to transport the NP downstream towards the hot fluid while thermophoresis now resists such migration. These counter effects lead to a local upstream accumulation of NP and their depletion downstream, at higher $\delta_{T}$ but lower $\delta_{n}$. Because of the NP accumulation, local stable positions are generated which in turn affect the growth of the instabilities, especially those of backward-growing fingers. Accordingly, initially unstable NP-laden CDH systems are less unstable in the presence of thermophoresis 
compared to the case without thermophoresis, although Brownian diffusivity still has destabilizing effects. Two counter-intuitive phenomena are found and discussed in both HDC and CDH systems. First, although Brownian diffusion and thermophoresis transport NP downstream synergically in the HDC system, local NP accumulation spots have been observed in the flow after a critical value of $\delta_{T}$. This was attributed to the advancement of the NP front downstream into the cold fluid region for large enough values of $\delta_{T}$. As a result, the viscosity profile becomes non-monotonic at large enough values of $\delta_{T}$ and $R_{n}$ which can result in the destabilization of initially stable HDC systems. Second, two regimes of instabilities are observed in the $\mathrm{CDH}$ case. For a range of small enough values of thermophoretic diffusivity, the flow instability was found to be attenuated with increasing $\delta_{T}$. These trends are however reversed beyond a certain critical value of $\delta_{T}$. Interestingly, these non-monotonic effects of $\delta_{T}$ on the instability in the $\mathrm{CDH}$ case are systematically accompanied with a suppression of the growth of backward-developing fingers as $\delta_{T}$ increases. This change in the trends was explained in terms of the development of regions resisting backward flow that can lead to an enhancement of the development of forward-growing fingers. One must note that, the general trends towards an attenuation of the instability are still observed in the presence of thermophoresis compared to the case without thermophoresis. It is also discussed that the NP accumulation due to the mechanisms discussed is intensified for low $\delta_{n}$ while it is mitigated under smooth temperature distributions, observed in particular at large Lewis numbers. Finally it is found that the main effects of Brownian diffusion and thermophoresis arise from their contribution to the NP transport while their contribution to the energy distribution can be safely disregarded.

In the third phase, the dispersed NPs had catalytic effects, causing the approaching fluids to react and produce another component in between. The approaching fluids A and B actually undergo the $A+B+n \rightarrow C+n$ reaction. This system was analyzed under both isothermal and non-isothermal conditions resulting from the heat of the reaction. In the isothermal case, as long as $R_{b}>R_{n}$ the flow is unstable after the reaction regardless of the 
value of $R_{c}$ while the stability condition is dependent on $R_{c}$ if $R_{b} \leq R_{n} . R_{b}, R_{c}$ and $R_{n}$ are the Log-viscosity ratios of the displaced fluid $\mathrm{B}$, the chemical products $\mathrm{C}$ and the nanofluid respectively to that of the displacing pure fluid A. Specifically, defining $R_{T}=R_{c}-R_{n}$ and $R_{L}=2 R_{b}-R_{c}-R_{n}$ as the effective viscosity ratios of the trailing and the leading zones respectively, the viscosity distribution is monotonically increasing if $R_{T}>0$ and $R_{L}>0$ while it is monotonically decreasing when $R_{T}<0$ and $R_{L}<0$. On the other hand its distribution may be non-monotonic if $R_{T} R_{L}<0$. The special case of $R_{b}=R_{c}$ reduces the problem to that of a non-reactive NP-laden displacement. The analysis showed that similar to non-reactive NP-laden systems, $R_{b}$ and $R_{n}$ has destabilizing and stabilizing effects respectively. However, the effect of $R_{c}$ on the instability is non-monotonic. The dynamics of the products then can be categorized based on the condition of the system before the reaction (whether it is intrinsically stable or unstable) and the effective viscosity ratios of the trailing and leading zones after the reaction. This allowed to identify six cases as Unstable (intrinsically unstable, both zones unstable after the reaction), IUD (intrinsically unstable, the leading zone unstable after the reaction), IUU (intrinsically unstable, the trailing zone unstable after the reaction), Stable (intrinsically stable, both zones stable after the reaction), $I S D$ (intrinsically stable, the leading zone unstable after the reaction) and ISU (intrinsically stable, the trailing zone unstable after the reaction).

The study reveals that in the presence of the heat of the chemical reaction that does not affect the mobility ratios, these stability conditions are no longer valid. This is in contrast with the NP-free systems where the dynamics of the flow in non-isothermal conditions are identical to those of isothermal systems if $R_{\theta}=0$. Further analysis shows that this behavior is a result of thermophoretic effects that interfere with the transport of NPs at large enough values of $H_{R}$ and $\delta_{T}$. The synergic effects of the heat of reaction and thermophoretic diffusivity can lead to an accumulation of NPs at both zones and their depletion at the center in both exothermic and endothermic reactions. The NP accumulation is more pronounced at the trailing zone where they are more abundant. Then with positive $R_{n}$ the viscosity dis- 
tribution and accordingly the transport of the chemical products are changed. Specifically, the viscosity is locally increased and decreased in the regions with accumulated or depleted NPs, respectively. The new viscosity distribution results in more developed or complex finger configurations in systems with unstable leading zone, the suppression of the backward product fingers, making stable system unstable and confining the chemical products in systems with the leading stable (or weakly unstable) zones. Accordingly, the center of mass of the products is affected compared to the isothermal case.

Regarding to the chemical products, in intrinsically unstable systems, the heat of reactionthermophoretic effects may have a non-monotonic behavior compared to cases where thermophoretic effects are not accounted for. Specifically, defining $\left(C_{c}\right) r_{i}=\frac{\left(C_{c}\right)_{t}\left(\delta_{T}=i\right)-\left(C_{c}\right) t\left(\delta_{T}=0\right)}{\left(C_{c}\right)_{t}\left(\delta_{T}=0\right)}$, in the $I U D$ and $U n$ stable systems $\left(C_{c}\right) r_{i}$ is first increasing with positive values in the passage of time, but later decreases and even becomes negative. However an opposite trend is observed in the $I U U$ systems. Depending on the choice of parameters the final value of $\left(C_{c}\right) r_{i}$ may not always be negative for the $I U D$ and Unstable or positive in the IUU systems. Differently, the response of intrinsically stable systems to the heat of reaction-thermophoretic effects are monotonic. In other words, in the $I S D$ and Stable systems $\left(C_{c}\right) r_{i} \geq 0$ while in the ISU system $\left(C_{c}\right) r_{i} \leq 0$.

The study shows that the presence of NPs has dramatic effects on the formation, growth and configuration of instability. However, many aspects are needed to be investigated to make the problem well understood. There is still a lack of knowledge about the deposition of NPs in porous media and so a complete model to predict its mechanism needs to be developed. A comprehensive model which includes many aspects of its mechanism may lead to new dynamics compared to the model used in this study. The addition of NPs to non-Newtonian fluids may result in different features in terms of the shear stress and so the viscosity. Very interesting dynamics are expected to be observed in this system. In terms of the flow in the non-isothermal condition, there is a great gap to find the stability criteria including all the parameters playing role beside the mobility ratios. The same question arises regarding nano- 
catalyst-laden systems both in the isothermal and non-isothermal conditions. A thorough investigation of the effect of NP deposition in the reactive systems is also recommended. In these systems the deposition not only does affect the viscosity of the nanofluid, but also it changes the reaction rate and the amount of the chemical products. The competition between these two factors may lead to interesting results. 


\section{Bibliography}

[1] P.G. Saffman and G.I. Taylor. The penetration of a fluid into a porous medium or heleshaw cell containing a more viscous liquid. Proceedings of the Royal Society of London A: Mathematical, Physical and Engineering Sciences, 245(1242):312-329, 1958.

[2] G. Taylor. The instability of liquid surfaces when accelerated in a direction perpendicular to their planes. i. In Proceedings of the Royal Society of London A: Mathematical, Physical and Engineering Sciences, volume 201, pages 192-196. The Royal Society, 1950.

[3] B. Jha, L. Cueto-Felgueroso, and R. Juanes. Fluid mixing from viscous fingering. Physical Review Letters, 106(19):194502, 2011.

[4] M. Mishra, P M. Trevelyan, C. Almarcha, and A. De Wit. Influence of double diffusive effects on miscible viscous fingering. Phys Rev Lett, 105(20):204501, 2010.

[5] U. G. Araktingi and FM. Orr Jr. Viscous fingering in heterogeneous porous media. SPE Advanced Technology Series, 1(01):71-80, 1993.

[6] M. N. Islam and J. Azaiez. Miscible thermo-viscous fingering instability in porous media. part 1: Linear stability analysis. Transport in Porous Media, 84(3):821-844, 2010.

[7] M. N. Islam and J. Azaiez. Miscible thermo-viscous fingering instability in porous 
media. part 2: Numerical simulations. Transport in Porous Media, 84(3):845-861, 2010.

[8] A. De Wit and G.M. Homsy. Viscous fingering in reaction-diffusion systems. The Journal of chemical physics, 110(17):8663-8675, 1999.

[9] A. De Wit and G.M. Homsy. Nonlinear interactions of chemical reactions and viscous fingering in porous media. Physics of fluids, 11(5):949-951, 1999.

[10] M. Sajjadi and J. Azaiez. Hydrodynamic instabilities of flows involving melting in under-saturated porous media. Physics of Fluids (1994-present), 28(3):033104, 2016.

[11] Q. Yuan and J. Azaiez. Miscible displacements in porous media with time-dependent injection velocities. Transport in Porous Media, 104(1):57-76, 2014.

[12] Ch.Y Chen and E. Meiburg. Miscible porous media displacements in the quarter fivespot configuration. part 1. the homogeneous case. Journal of Fluid Mechanics, 371: 233-268, 1998.

[13] R. Saidur, K..Y Leong, and H.A. Mohammad. A review on applications and challenges of nanofluids. Renewable and sustainable energy reviews, 15(3):1646-1668, 2011. ISSN 1364-0321.

[14] B.A. Suleimanov, F.S. Ismailov, and E.F. Veliyev. Nanofluid for enhanced oil recovery. Journal of Petroleum Science and Engineering, 78(2):431-437, 2011.

[15] L. Hendraningrat, Sh. Li, and O. Torsæter. A coreflood investigation of nanofluid enhanced oil recovery. Journal of Petroleum Science and Engineering, 111:128-138, 2013.

[16] L.M. Ensign, R. Cone, and J. Hanes. Oral drug delivery with polymeric nanoparticles: The gastrointestinal mucus barriers. Advanced Drug Delivery Reviews, 64(6):557-570, 2012. 
[17] H.A. Stone, A.D. Stroock, and A. Ajdari. Engineering flows in small devices: microfluidics toward a lab-on-a-chip. Annu. Rev. Fluid Mech., 36:381-411, 2004.

[18] R. Hashemi and P. Nassar, N.and Pereira Almao. Enhanced heavy oil recovery by in situ prepared ultradispersed multimetallic nanoparticles: A study of hot fluid flooding for athabasca bitumen recovery. Energy and Fuels, 27(4):2194-2201, 2013.

[19] N.C. Mueller and B. Nowack. Nanoparticles for remediation: Solving big problems with little particles. Elements, 6(6):395-400, 2010.

[20] S. Hill. Channeling in packed columns. Chemical Engineering Science, 1(6):247-253, 1952.

[21] R.L. Chuoke, P. van Meurs, and C. van der Poel. The instability of slow, immiscible, viscous liquid-liquid displacements in permeable media. SPE, 216:188-194, 1959.

[22] C.T. Tan and G.M. Homsy. Stability of miscible displacements in porous media: rectilinear flow. Physics of Fluids (1958-1988), 29(11):3549-3556, 1986.

[23] D.W. Peaceman and H.H. Rachford Jr. Numerical calculation of multidimensional miscible displacement. Society of Petroleum Engineers Journal, 2(04):327-339, 1962.

[24] D.T. Hatziavramids. Proceedings of the society of petroleum engineers. SPE, page 19004, 1987.

[25] C.T. Tan and G.M. Homsy. Simulation of nonlinear viscous fingering in miscible displacement. Physics of Fluids (1958-1988), 31(6):1330-1338, 1988.

[26] W.B. Zimmerman and G.M. Homsy. Three dimensional viscous fingering: A numerical study. Physics of Fluids A: Fluid Dynamics (1989-1993), 4(9):1901-1914, 1992.

[27] J.S. Nijjer, D.R. Hewitt, and J.A. Neufeld. The dynamics of miscible viscous fingering from onset to shutdown. Journal of Fluid Mechanics, 837:520-545, 2018. 
[28] L.L. Handy. An evaluation of diffusion effects in miscible displacement. Journal of Petroleum Technology, 11(03):61-63, 1959.

[29] R.L. Slobod and R.A. Thomas. Effect of transverse diffusion on fingering in misciblephase displacement. Society of Petroleum Engineers Journal, 3(01):9-13, 1963.

[30] W.B. Zimmerman and G.M. Homsy. Nonlinear viscous fingering in miscible displacement with anisotropic dispersion. Physics of Fluids A: Fluid Dynamics, 3(8):1859$1872,1991$.

[31] W.B. Zimmerman and G.M. Homsy. Viscous fingering in miscible displacements: Unification of effects of viscosity contrast, anisotropic dispersion, and velocity dependence of dispersion on nonlinear finger propagation. Physics of Fluids A: Fluid Dynamics, 4 (11):2348-2359, 1992.

[32] O. Manickam and G.M. Homsy. Stability of miscible displacements in porous media with nonmonotonic viscosity profiles. Physics of Fluids A: Fluid Dynamics, 5(6):13561367, 1993.

[33] K. Ghesmat and J. Azaiez. Viscous fingering instability in porous media: effect of anisotropic velocity-dependent dispersion tensor. Transport in Porous Media, 73(3): 297-318, 2008.

[34] J.R. Waggoner, J.L. Castillo, and L.W. Lake. Simulation of eor processes in stochastically generated permeable media. SPE formation evaluation, 7(02):173-180, 1992.

[35] R.J. Blackwell, J.R. Rayne, and W.M. Terry. Factors influencing the efficiency of miscible displacement. SPE, 1959.

[36] U.G. Araktingi. Viscous fingering in heterogeneous porous media. PhD thesis, Stanford University, 1988. 
[37] C.T. Tan and G.M. Homsy. Viscous fingering with permeability heterogeneity. Physics of Fluids A: Fluid Dynamics, 4(6):1099-1101, 1992.

[38] H.A. Tchelepi, F.M. Orr Jr, N. Rakotomalala, D. Salin, and R. Woumeni. Dispersion, permeability heterogeneity, and viscous fingering: Acoustic experimental observations and particle-tracking simulations. Physics of Fluids A: Fluid Dynamics, 5(7):15581574, 1993.

[39] A. De Wit and G.M. Homsy. Viscous fingering in periodically heterogeneous porous media. i. formulation and linear instability. The Journal of chemical physics, 107(22): 9609-9618, 1997.

[40] A. De Wit and G.M. Homsy. Viscous fingering in periodically heterogeneous porous media. ii. numerical simulations. The Journal of chemical physics, 107(22):9619-9628, 1997.

[41] M. Norouzi and M.R. Shoghi. A numerical study on miscible viscous fingering instability in anisotropic porous media. Physics of Fluids, 26(8):084102, 2014.

[42] D. Pritchard. The instability of thermal and fluid fronts during radial injection in a porous medium. Journal of Fluid Mechanics, 508:133-163, 2004.

[43] J. Azaiez and M. Sajjadi. Stability of double-diffusive double-convective miscible displacements in porous media. Phys Rev E Stat Nonlin Soft Matter Phys, 85(2 Pt 2): 026306, 2012.

[44] V. Hornof and F.U. Baig. Influence of interfacial reaction and mobility ratio on the displacement of oil in a hele-shaw cell. Experiments in Fluids, 18(6):448-453, 1995.

[45] M.L. Dickson, T.T Norton, and E.J. Fernandez. Chemical imaging of multicomponent viscous fingering in chromatography. AIChE Journal, 43(2):409-418, 1997. 
[46] J.A. Cunningham and Z.J. Fadel. Contaminant degradation in physically and chemically heterogeneous aquifers. Journal of Contaminant Hydrology, 94(3):293-304, 2007.

[47] J.A. Pojman, G. Gunn, C. Patterson, J. Owens, and C. Simmons. Frontal dispersion polymerization. The Journal of Physical Chemistry B, 102(20):3927-3929, 1998.

[48] S. Swernath and S. Pushpavanam. Viscous fingering in a horizontal flow through a porous medium induced by chemical reactions under isothermal and adiabatic conditions. The Journal of chemical physics, 127(20):204701, 2007.

[49] Y. Nagatsu, K. Matsuda, Y. Kato, and Y. Tada. Experimental study on miscible viscous fingering involving viscosity changes induced by variations in chemical species concentrations due to chemical reactions. Journal of Fluid Mechanics, 571:475-493, 2007.

[50] Y. Nagatsu, C. Iguchi, K. Matsuda, Y. Kato, and Y. Tada. Miscible viscous fingering involving viscosity changes of the displacing fluid by chemical reactions. Physics of Fluids, 22(2):024101, 2010.

[51] T. Podgorski, M.C. Sostarecz, S. Zorman, and A. Belmonte. Fingering instabilities of a reactive micellar interface. Physical Review E, 76(1):016202, 2007.

[52] T. Gérard and A. De Wit. Miscible viscous fingering induced by a simple $A+B \rightarrow C$ chemical reaction. Physical Review E, 79(1):016308, 2009.

[53] S.H. Hejazi, P.M.J. Trevelyan, J. Azaiez, and A. De Wit. Viscous fingering of a miscible reactive $A+B \rightarrow C$ interface: a linear stability analysis. Journal of fluid mechanics, 652:501-528, 2010.

[54] S.H. Hejazi and J. Azaiez. Non-linear interactions of dynamic reactive interfaces in porous media. Chemical Engineering Science, 65(2):938-949, 2010. 
[55] Y. Nagatsu and A. De Wit. Viscous fingering of a miscible reactive $A+B \rightarrow C$ interface for an infinitely fast chemical reaction: Nonlinear simulations. Physics of fluids, 23(4):043103, 2011.

[56] S.M.S. Murshed and P. Estellé. A state of the art review on viscosity of nanofluids. Renewable and Sustainable Energy Reviews, 76:1134-1152, 2017.

[57] M.H. Ahmadi, A. Mirlohi, M.A. Nazari, and R. Ghasempour. A review of thermal conductivity of various nanofluids. Journal of Molecular Liquids, 265:181 - 188, 2018.

[58] K.H. Solangi, S.N. Kazi, M.R. Luhur, A. Badarudin, A. Amiri, R. Sadri, M.N.M. Zubir, S. Gharehkhani, and K.H. Teng. A comprehensive review of thermo-physical properties and convective heat transfer to nanofluids. Energy, 89:1065-1086, 2015.

[59] B.D Roussennac and C. Toschi. Brightwater trial in salema field (campos basin, brazil). In SPE EUROPEC/EAGE annual conference and exhibition. Society of Petroleum Engineers, 2010.

[60] J. Wu, J. He, O. Torsater, and Z. Zhang. Effect of nanoparticles on oil-water flow in a confined nanochannel: a molecular dynamics study. In SPE International Oilfield Nanotechnology Conference and Exhibition. Society of Petroleum Engineers, 2012.

[61] D.T. Wasan and A.D. Nikolov. Spreading of nanofluids on solids. Nature, 423(6936): $156-159,2003$.

[62] K. Kondiparty, A.D. Nikolov, D. Wasan, and K.L Liu. Dynamic spreading of nanofluids on solids. part i: experimental. Langmuir, 28(41):14618-14623, 2012.

[63] K. Ghesmat, H. Hassanzadeh, J. Abedi, and Z. Chen. Influence of nanoparticles on the dynamics of miscible hele-shaw flows. Journal of Applied Physics, 109(10):104907, 2011. 
[64] K. Ghesmat, H. Hassanzadeh, J. Abedi, and Z. Chen. Frontal stability of reactive nanoparticle transport during in situ catalytic upgrading of heavy oil. Fuel, 107:525$538,2013$.

[65] M. Zargartalebi and J. Azaiez. Mesoscopic study of miscible nanoflow instabilities. Physics of Fluids, 30(2):024105, 2018.

[66] N. Sabet, S.M. Jafari Raad, H. Hassanzadeh, and J. Abedi. Dynamics of miscible nanocatalytic reactive flows in porous media. Physical Review Applied, 10(5):054033, 2018.

[67] K.M. Yao, M.T. Habibian, and C.R. O’Melia. Water and waste water filtration. concepts and applications. Environmental science and technology, 5(11):1105-1112, 1971.

[68] C. Tien and B.V. Ramarao. Granular filtration of aerosols and hydrosols. Elsevier, 2011.

[69] S. Lakshmanan, W.M. Holmes, W.T. Sloan, and V.R. Phoenix. Nanoparticle transport in saturated porous medium using magnetic resonance imaging. Chemical Engineering Journal, 266:156-162, 2015.

[70] O. Manickam and G.M. Homsy. Simulation of viscous fingering in miscible displacements with nonmonotonic viscosity profiles. Physics of Fluids (1994-present), 6(1): 95-107, 1994.

[71] P.M.J. Trevelyan, C. Almarcha, and A. De Wit. Buoyancy-driven instabilities of miscible two-layer stratifications in porous media and hele-shaw cells. Journal of fluid mechanics, 670:38-65, 2011.

[72] S.M. Jafari Raad, H. Emami Meybodi, and H. Hassanzadeh. On the choice of analogue fluids in CO2 convective dissolution experiments. Water Resources Research, 52(6): 4458-4468, 2016. 
[73] S.M. Jafari Raad and H. Hassanzadeh. Onset of dissolution-driven instabilities in fluids with nonmonotonic density profile. Physical Review E, 92(5):053023, 2015.

[74] S.M. Jafari Raad and H. Hassanzadeh. Does impure CO2 impede or accelerate the onset of convective mixing in geological storage? International Journal of Greenhouse Gas Control, 54, Part 1:250-257, 2016.

[75] M.C. Cross and P.C. Hohenberg. Pattern formation outside of equilibrium. Reviews of modern physics, 65(3):851, 1993.

[76] Y.H. Shokrlu and T. Babadagli. Viscosity reduction of heavy oil/bitumen using microand nano-metal particles during aqueous and non-aqueous thermal applications. Journal of Petroleum Science and Engineering, 119:210-220, 2014.

[77] M.N. Islam and J. Azaiez. Fully implicit finite difference pseudo-spectral method for simulating high mobility-ratio miscible displacements. International journal for numerical methods in fluids, 47(2):161-183, 2005.

[78] B.K. Singh. Flow of Newtonian and non-Newtonian fluids in porous media: the viscous fingering instability. Calgary, 2000. ISBN 0612496856.

[79] J.C. Giddings. Field-flow fractionation: analysis of macromolecular, colloidal, and particulate materials. Science, 260(5113):1456-1465, 1993.

[80] J. Wen and A.S. Wexler. Thermophoretic sampler and its application in ultrafine particle collection. Aerosol science and technology, 41(6):624-629, 2007.

[81] R. Golestanian, T.B. Liverpool, and A. Ajdari. Designing phoretic micro-and nanoswimmers. New Journal of Physics, 9(5):126, 2007.

[82] R. Hashemi, N.N. Nassar, and P. Pereira Almao. Nanoparticle technology for heavy oil in-situ upgrading and recovery enhancement: Opportunities and challenges. Applied Energy, 133:374-387, 2014. 
[83] W.M. Deen. Analysis of Transport Phenomena. Oxford University Press, New York, 1998.

[84] J. Buongiorno. Convective transport in nanofluids. Journal of Heat Transfer, 128(3): 240-250, 2006.

[85] D.A. Nield and A.V. Kuznetsov. Thermal instability in a porous medium layer saturated by a nanofluid. International Journal of Heat and Mass Transfer, 52(25):5796$5801,2009$.

[86] N. Tufenkji and M. Elimelech. Correlation equation for predicting single-collector efficiency in physicochemical filtration in saturated porous media. Environmental science and technology, 38(2):529-536, 2004.

[87] T. Zhang. Modeling of nanoparticle transport in porous media. PhD thesis, The University of Texas at Austin, 2012.

[88] T. Zhang, M. Murphy, H. Yu, C. Huh, and S.L. Bryant. Mechanistic model for nanoparticle retention in porous media. Transport in Porous Media, 115(2):387-406, 2016.

[89] E.E. Michaelides. Brownian movement and thermophoresis of nanoparticles in liquids. International Journal of Heat and Mass Transfer, 81:179-187, 2015.

[90] G.S. McNab and A. Meisen. Thermophoresis in liquids. Journal of Colloid and Interface Science, 44(2):339-346, 1973.

[91] A. Martin and M.M. Bou-Ali. Determination of thermal diffusion coefficient of nanofluid: Fullerene-toluene. Comptes Rendus Mécanique, 339(5):329-334, 2011.

[92] J. Gargiulo, S. Cerrota, E. Cortés, I.L. Violi, and F.D. Stefani. Connecting metallic nanoparticles by optical printing. Nano letters, 16(2):1224-1229, 2016.

[93] R. Piazza and A. Parola. Thermophoresis in colloidal suspensions. Journal of Physics: Condensed Matter, 20(15):153102, 2008. 
[94] M. Braibanti, D. Vigolo, and R. Piazza. Does thermophoretic mobility depend on particle size? Physical review letters, 100(10):108303, 2008.

[95] A. Zaraki, M. Ghalambaz, A.J. Chamkha, M. Ghalambaz, and D. De Rossi. Theoretical analysis of natural convection boundary layer heat and mass transfer of nanofluids: effects of size, shape and type of nanoparticles, type of base fluid and working temperature. Advanced Powder Technology, 26(3):935-946, 2015.

[96] W. Yu and H. Xie. A review on nanofluids: preparation, stability mechanisms, and applications. Journal of nanomaterials, 2012:1, 2012.

[97] S. Malhotra, M.M. Sharma, and E.R Lehman. Experimental study of the growth of mixing zone in miscible viscous fingering. Physics of Fluids, 27(1):014105, 2015.

[98] S.M. Shuwa, R.S. Al-Hajri, A. Mohsenzadeh, Y.M. Al-Waheibi, and B.Y. Jibril. Heavy crude oil recovery enhancement and in-situ upgrading during steam injection using nico-mo dispersed catalyst. In SPE EOR Conference at Oil and Gas West Asia, page 17. Society of Petroleum Engineers, 2016.

[99] J.B. Omajali, A. Hart, M. Walker, J. Wood, and L.E. Macaskie. In-situ catalytic upgrading of heavy oil using dispersed bionanoparticles supported on gram-positive and gram-negative bacteria. Applied Catalysis B: Environmental, 203:807-819, 2017.

[100] M.C Chang, H.Y. Shu, W.P. Hsieh, and M.C. Wang. Using nanoscale zero-valent iron for the remediation of polycyclic aromatic hydrocarbons contaminated soil. Journal of the Air and Waste Management Association, 55(8):1200-1207, 2005.

[101] P.L. Freund and M. Spiro. Colloidal catalysis: the effect of sol size and concentration. The Journal of Physical Chemistry, 89(7):1074-1077, 1985.

[102] H.N. McMurray. Particle size effects in electrocatalysis by uniform colloids of ruthenium dioxide hydrate. The Journal of Physical Chemistry, 98(39):9861-9864, 1994. 
[103] S. Carregal-Romero, J. Pérez-Juste, P. Hervés, L.M. Liz-Marzán, and P. Mulvaney. Colloidal gold-catalyzed reduction of ferrocyanate (iii) by borohydride ions: a model system for redox catalysis. Langmuir, 26(2):1271-1277, 2009.

[104] Yin Li, Xiaoyong M Hong, David M Collard, and Mostafa A El-Sayed. Suzuki crosscoupling reactions catalyzed by palladium nanoparticles in aqueous solution. Organic letters, 2(15):2385-2388, 2000.

[105] B. Dastvareh and J. Azaiez. Thermophoretic effects on instabilities of nanoflows in porous media. Journal of Fluid Mechanics, 857:173-199, 2018.

[106] B. Dastvareh and J. Azaiez. Instabilities of nanofluid flow displacements in porous media. Physics of Fluids, 29(4):044101, 2017. 


\section{Appendix A}

\section{Derivation of the Linear Disturbed}

\section{Equations}

In order to conduct linear stability analysis, small disturbances are introduced to the system:

$$
\left(u, v, P, C_{i}, \mu\right)(x, y, t)=\left(\bar{u}, \bar{v}, \bar{p}, \bar{C}_{i}, \bar{\mu}\right)(x, t)+\left(u^{\prime}, v^{\prime}, p^{\prime}, C_{i}^{\prime}, \mu^{\prime}\right)(x, y, t)
$$

Where $\mu^{\prime}=C_{a}{ }^{\prime} \frac{\partial \bar{\mu}}{\partial \bar{C}_{a}}+C_{b}{ }^{\prime} \frac{\partial \bar{\mu}}{\partial \bar{C}_{b}}+C_{n}{ }^{\prime} \frac{\partial \bar{\mu}}{\partial \bar{C}_{n}}$. By neglecting the higher order perturbations the resulting linear disturbed equations are:

$$
\begin{gathered}
\frac{\partial u^{\prime}}{\partial x}+\frac{\partial v^{\prime}}{\partial y}=0 \\
\frac{\partial P^{\prime}}{\partial x}=-\bar{\mu} u^{\prime}-\mu^{\prime}, \quad \frac{\partial P^{\prime}}{\partial y}=-\bar{\mu} v^{\prime} \\
\frac{\partial C_{a}{ }^{\prime}}{\partial t}+u^{\prime} \frac{\partial \bar{C}_{a}}{\partial x}=\frac{\partial^{2} C_{a}{ }^{\prime}}{\partial x^{2}}+\frac{\partial^{2} C_{a}{ }^{\prime}}{\partial y^{2}} \\
\frac{\partial C_{b}{ }^{\prime}}{\partial t}+u^{\prime} \frac{\partial \bar{C}_{b}}{\partial x}=\delta_{b}\left(\frac{\partial^{2} C_{b}{ }^{\prime}}{\partial x^{2}}+\frac{\partial^{2} C_{b}{ }^{\prime}}{\partial y^{2}}\right) \\
\frac{\partial C_{n}{ }^{\prime}}{\partial t}+u^{\prime} \frac{\partial \bar{C}_{n}}{\partial x}=\delta_{n}\left(\frac{\partial^{2} C_{n}{ }^{\prime}}{\partial x^{2}}+\frac{\partial^{2} C_{n}{ }^{2}}{\partial y^{2}}\right)-D a_{d e p} C_{n}{ }^{\prime}
\end{gathered}
$$

By taking the curl of Darcy's law the pressure is eliminated from the equations. This 
leads to the following equation:

$$
-\frac{\partial}{\partial x}\left(\bar{\mu} v^{\prime}\right)+\frac{\partial}{\partial y}\left(\bar{\mu} u^{\prime}+\mu^{\prime}\right)=0
$$

Or:

$$
\bar{\mu}\left(\frac{\partial v^{\prime}}{\partial x}-\frac{\partial u^{\prime}}{\partial y}\right)-\frac{\partial \mu^{\prime}}{\partial y}+v^{\prime} \frac{\partial \bar{\mu}}{\partial x}=0
$$

By taking derivative from Eq. A.8 with respect to y and using the continuity equation, the dependency of this equation on $v^{\prime}$ will be eliminated.

$$
\frac{\partial^{2} u^{\prime}}{\partial x^{2}}+\frac{\partial^{2} u^{\prime}}{\partial y^{2}}+\frac{1}{\bar{\mu}} \frac{\partial^{2} \mu^{\prime}}{\partial y^{2}}+\frac{1}{\bar{\mu}} \frac{\partial u^{\prime}}{\partial x} \frac{\partial \bar{\mu}}{\partial x}=0
$$

Where

$$
\frac{1}{\bar{\mu}} \frac{\partial \bar{\mu}}{\partial x}=R_{a} \frac{\partial \bar{C}_{a}}{\partial x}+R_{b} \frac{\partial \bar{C}_{b}}{\partial x}+R_{n} \frac{\partial \bar{C}_{n}}{\partial x}
$$

Eq. A.9 and A.4-A.6 are the final linear disturbed equations that would be decomposed in terms of Fourier components in the next stage. 


\section{Appendix B}

\section{Derivation of the Characteristic}

\section{Equations for Sharp Front}

By assuming $\frac{d \bar{C}_{a}}{d x}=-\frac{d \bar{C}_{b}}{d x}=-\delta(x)$ and $\frac{d \bar{C}_{n}}{d x}=-C_{n 0} \delta(x)$ the results are the following set of equations:

$$
\begin{gathered}
\frac{d^{2} \phi}{d x^{2}}-l^{2} \phi=-Y \delta(x) \\
\frac{d^{2} \chi}{d x^{2}}-p^{2} \chi=\frac{Y \delta(x)}{\delta_{b}} \\
\frac{d^{2} \psi}{d x^{2}}-q^{2} \psi=-\frac{Y C_{n 0} \delta(x)}{\delta_{n}} \\
\frac{d^{2} Y}{d x^{2}}+\left(R_{b}-R_{a}-C_{n 0} R_{n}\right) \delta(x) \frac{d Y}{d x}-k^{2} Y=k^{2}\left(R_{a} \phi+R_{b} X+R_{n} \psi\right)
\end{gathered}
$$

Where:

$$
\begin{gathered}
l^{2}=k^{2}+\gamma \\
p^{2}=k^{2}+\frac{\gamma}{\delta_{b}} \\
q^{2}=k^{2}+\frac{\gamma+D a_{d e p}}{\delta_{n}}
\end{gathered}
$$

By solving the above set of equations for $x$ away from the interface one gets: 


$$
\begin{gathered}
\phi(x)=A_{1} \exp (l x) \quad x<0, \\
\phi(x)=A_{2} \exp (-l x) \quad x>0 \\
\chi(x)=B_{1} \exp (p x) \quad x<0, \\
\chi(x)=B_{2} \exp (-p x) \quad x>0 \\
\psi(x)=C_{1} \exp (q x) \quad x<0, \\
\psi(x)=C_{2} \exp (-q x) \quad x>0 \\
Y(x)=a A_{1} \exp (l x)+b B_{1} \exp (p x)+c C_{1} \exp (q x)+D_{1} \exp (k x) \quad x<0, \\
Y(x)=a A_{2} \exp (-l x)+b B_{2} \exp (-p x)+c C_{2} \exp (-q x)+D_{2} \exp (-k x) \quad x>0
\end{gathered}
$$

Where:

$$
a=\frac{k^{2} R_{a}}{\gamma}, \quad b=\frac{k^{2} R_{b} \delta_{b}}{\gamma}, \quad c=\frac{k^{2} R_{n} \delta_{n}}{\gamma+D a_{d e p}}
$$

Equation B.6-B.9 has eight unknowns which shows that eight conditions are needed to solve it. By using the condition of continuity of the concentrations and velocity at $x=0$, jump in the normal stress and integration of concentration equations (Eq. B.6 to B.8) from $0^{-}$to $0^{+}$, the following equations are obtained:

$$
\begin{array}{r}
(1+\alpha)\left(a l A_{1}+b p B_{1}+c q C_{1}+k D_{1}\right)=0 \\
(a-2 l) A_{1}+b B_{1}+c C_{1}+D_{1}=0 \\
a A_{1}+\left(b+2 \delta_{b} p\right) B_{1}+c C_{1}+D_{1}=0 \\
a A_{1}+b B_{1}+\left(c-\frac{2 \delta_{n} q}{C_{n 0}}\right) C_{1}+D_{1}=0
\end{array}
$$

Where $\alpha=\frac{\mu\left(0^{+}\right)}{\mu\left(0^{-}\right)}=\exp \left(R_{b}-R_{a}-C_{n 0} R_{n}\right)$. Other four unknowns $\left(A_{2}, B_{2}, C_{2}, D_{2}\right)$ are equal to their pairs $\left(A_{1}, B_{1}, C_{1}, D_{1}\right)$. For non-trivial solutions, the determinant of the matrix containing the coefficients of the above equations must be zero. This leads to: 


$$
(1+\alpha)\left(p q \delta_{b} \delta_{n}(a k-2 l k-a l)+C_{n 0} p c l \delta_{b}(k-q)+q b l \delta_{n}(p-k)\right)=0
$$

Dividing both sides by $(1+\alpha)$ and substituting $a, b, c, l, p$ and $q$ Eq. 3.16 is derived. 


\section{Appendix C}

\section{Stability Criteria and Characteristic Equation for Intrinsically Stable Displacement}

From Eq. 3.23 it is concluded that

$$
\begin{gathered}
k^{2}+\gamma=0 \\
-2 \sqrt{\delta_{n} k^{2}+\gamma+D a_{d e p}}+\frac{k R_{n}}{\gamma+D a_{\text {dep }}}\left(k \sqrt{\delta_{n}}-\sqrt{\delta_{n} k^{2}+\gamma+D a_{\text {dep }}}\right)=0
\end{gathered}
$$

Accordingly, $\gamma=-k^{2}$ can be an answer. Setting $Y=\sqrt{\delta_{n} k^{2}+\gamma+D a_{d e p}}$ and after some algebra, Eq. C.2 can be written as follows:

$$
\left(k \sqrt{\delta_{n}}-Y\right)\left(2 Y^{2}+2 Y k \sqrt{\delta_{n}}+k R_{n}\right)=0
$$


Solving the above equation results in the following roots for $\mathrm{Y}$ :

$$
\begin{gathered}
Y=k \sqrt{\delta_{n}} \\
Y=\frac{-k \sqrt{\delta_{n}}}{2}+\frac{1}{2} \sqrt{\delta_{n} k^{2}-2 k R_{n}} \\
Y=\frac{-k \sqrt{\delta_{n}}}{2}-\frac{1}{2} \sqrt{\delta_{n} k^{2}-2 k R_{n}}
\end{gathered}
$$

Since $Y$ must be positive, Eq. C.4c is not acceptable. Eq. C.4a also cannot be an acceptable solution as after substituting $Y$, it is concluded that $\gamma=-D a_{d e p}$, which is not acceptable considering Eq. C.2. Accordingly, simplification of Eq. C.4b results in the following equation for the growth rate:

$$
\gamma=-\frac{1}{2} \delta_{n} k^{2}-\frac{1}{2} k R_{n}-D a_{d e p}-\frac{k}{2} \sqrt{\delta_{n}^{2} k^{2}-2 \delta_{n} R_{n} k}
$$

Eq. C.5 is composed of two parts: with and without square root. For $R_{n}>0$ the first part (without square root) is always negative. The second part can be a real negative number or a pure imaginary one $\left(k \leq \frac{2 R_{n}}{\delta_{n}}\right)$. Since the real part of Eq. C.5 is always negative the flow is stable for $R_{n}>0$. For $R_{n} \leq 0$ depending on the value of $-\frac{1}{2} k R_{n}$ with respect to the other terms, the flow can be stable or unstable and the criteria of stability will be discussed after finding the cut-off wave number. For $R_{n} \leq 0$, the dispersion curve should be composed of Eq. C.5 and/or $\gamma=-k^{2}$. It can be easily shown that the long wave expansion of Eq. 3.23 is in the form of $\gamma=-k^{2}+o\left(k^{2}\right)$. Accordingly, and since Eq. C.5 is not valid for $k=0$, it can be concluded that in the range of $0 \leq k \leq k_{c r}$ the dispersion curve is $\gamma=-k^{2}$ and for $k \geq k_{c r}$ it obeys Eq. C.5. To find $k_{c r}$, equating Eq. C.5 and $\gamma=-k^{2}$ leads to:

$$
k \sqrt{\delta_{n}^{2} k^{2}-2 \delta_{n} R_{n} k}=k^{2}\left(2-\delta_{n}\right)-k R_{n}-2 D a_{d e p}
$$


After some algebra the following equation is derived to determine $k_{c r}$ :

$$
4\left(1-\delta_{n}\right) k^{4}-4\left(1-\delta_{n}\right) R_{n} k^{3}+\left(R_{n}^{2}+4\left(\delta_{n}-2\right) D a_{d e p}\right) k^{2}+4 R_{n} D a_{d e p} k+4 D a_{d e p}^{2}=0
$$

Eq. C.7 has in general four roots that can be obtained analytically. The acceptable real positive root must obey the condition $k^{2}\left(2-\delta_{n}\right)-k R_{n}-2 D a_{d e p} \geq 0$ resulting from Eq. C.6. The aim here is to find the dispersion curve in unstable situations. So, it must be noted that since the dispersion curves must be type 1 , if more than one real positive root satisfies the mentioned condition, the smallest one among them is $k_{c r}$. For the case of $\delta_{n}=1$, Eq. C.7 reduces to a second order equation and $k_{c r}$ can be easily found in this case. To find the condition of instability the following procedure is conducted.

Substituting $\gamma=0$ into Eq. C.5 one reaches:

$$
k \sqrt{\delta_{n}^{2} k^{2}-2 \delta_{n} R_{n} k}=-\left(\delta_{n} k^{2}+k R_{n}+2 D a_{d e p}\right)
$$

After some algebra, the cut off wave number can be found form the flowing equation:

$$
4 \delta_{n} R_{n} k^{3}+\left(R_{n}^{2}+4 \delta_{n} D a_{d e p}\right) k^{2}+4 D a_{d e p} R_{n} k+4 D a_{d e p}^{2}=0
$$

Considering Eq. C.8, the acceptable $k$ must satisfy the following condition:

$$
\delta_{n} k^{2}+k R_{n}+2 D a_{d e p} \leq 0
$$

Eq. C.9 has analytical solution. Based on the properties of NPs one has three scenarios: 1. Eq. C.9 has three distinct real roots. Among these roots one of them will not satisfy Eq. C.10 and the remaining roots are the acceptable roots. In this situation the flow is unstable and the larger positive real root is the cut off wave number. It is well-known that the criteria for a cubic equation of $a x^{3}+b x^{2}+c x+d=0$ to have a three distinct real roots 
is $18 a b c d-4 b^{3} d+b^{2} c^{2}-4 a c^{3}-27 a^{2} d^{2}>0$. Accordingly, and after some algebra one can reach to following condition for the instability:

$$
R_{n}<-\sqrt{4(5.5+2.5 \sqrt{5}) \delta_{n} D a_{d e p}}
$$

2. Eq. C.9 has one real root and two complex roots. This real root will not satisfy Eq. C.9 and as a result the system is stable.

3. Eq. C.9 has multiple roots. In this situation the flow is stable. 


\section{Appendix D}

\section{Solving the non-linear equations with pseudo-spectral method}

In this section the procedure to solve Eq. 3.28 is described briefly. The similar procedure is implemented to solve Eqs. 4.24 and 5.18.

Hartley introduced the following direct and inverse transforms in 1942:

$$
\begin{gathered}
\hat{g}\left(k_{x}\right)=\frac{1}{\sqrt{2 \pi}} \int_{-\infty}^{+\infty} g(x)\left[\cos \left(k_{x} x\right)+\sin \left(k_{x} x\right)\right] d x \\
g(x)=\frac{1}{\sqrt{2 \pi}} \int_{-\infty}^{+\infty} \hat{g}\left(k_{x}\right)\left[\cos \left(k_{x} x\right)+\sin \left(k_{x} x\right)\right] d\left(k_{x}\right)
\end{gathered}
$$

Where $k_{x}$ and $k_{y}$ are the wave numbers in the $x$ and $y$ directions. For a real function $g(x)$, the wave numbers in the transform space is real. As a result, one only deal with real numbers by implementing Hartley transform. 
The derivatives of a function in Hartley space are in the following form:

$$
\begin{gathered}
H\left[\frac{\partial}{\partial x} g(x, y, t)\right]=-k_{x} \hat{g}\left(-k_{x},-k_{y}, t\right) \\
H\left[\frac{\partial}{\partial y} g(x, y, t)\right]=-k_{y} \hat{g}\left(-k_{x},-k_{y}, t\right) \\
H\left[\frac{\partial^{2}}{\partial x^{2}} g(x, y, t)\right]=-k_{x}{ }^{2} \hat{g}\left(k_{x}, k_{y}, t\right) \\
H\left[\frac{\partial^{2}}{\partial y^{2}} g(x, y, t)\right]=-k_{y}{ }^{2} \hat{g}\left(k_{x}, k_{y}, t\right)
\end{gathered}
$$

Accordingly, Eq. 3.28 is transformed to the Hartley space as below:

$$
\begin{gathered}
\frac{\partial \hat{C}_{a}}{\partial t}=\hat{J}_{a}-\left(k_{x}{ }^{2}+k_{y}{ }^{2}\right) \hat{C}_{a} \\
\frac{\partial \hat{C}_{b}}{\partial t}=\hat{J}_{b}-\delta_{b}\left(k_{x}{ }^{2}+k_{y}{ }^{2}\right) \hat{C}_{b} \\
\frac{\partial \hat{C}_{n}}{\partial t}=\hat{J}_{n}-\left[\delta_{n}\left(k_{x}{ }^{2}+k_{y}{ }^{2}\right)-D a_{d e p}\right] \hat{C}_{n} \\
\hat{\omega}=R_{a} \hat{N}_{a}+R_{b} \hat{N}_{b}+R_{n} \hat{N}_{n} \\
\hat{\omega}=\left(k_{x}{ }^{2}+k_{y}{ }^{2}\right) \hat{\psi}
\end{gathered}
$$

Note that $\hat{C}_{i}, \hat{\omega}, \hat{\psi}, \hat{J}_{i}, \hat{N}_{i}$ are the Hartley transform functions of $C_{i}, \omega, \psi, J_{i}, N_{i}$ respectively. The ODEs in Eq. D.7 are actually in the following form:

$$
\frac{\partial \hat{C}}{\partial t}=\hat{J}-\lambda^{2} \hat{C}
$$

Assuming $\hat{C}=\tilde{C} e^{-\lambda^{2} t}$ and after some manipulations, one can reach to:

$$
\frac{\partial \tilde{C}}{\partial t}=\tilde{J}
$$

Where $\tilde{J}=\hat{J} e^{\lambda^{2} t}$. To find the concentrations in the next step a two-step semi implicit AdamBashforth, Adam-Molton predictor-corrector method is used. In this scheme first the rough 
approximation of the desired concentrations are calculated by Adam-Bashforth method:

$$
\tilde{C}^{p}(t+\Delta t)=\tilde{C}^{p}(t)+\frac{\Delta t}{2}(3 \tilde{J}(t)-\tilde{J}(t-\Delta t))
$$

Or after substituting $\tilde{J}(t)=\hat{J}(t) e^{\lambda^{2} t}$ and $\tilde{C}(t)=\hat{C}(t) e^{\lambda^{2} t}$ :

$$
\hat{C}^{p}(t+\Delta t)=\left[\hat{C}^{p}(t)+\frac{\Delta t}{2}\left(3 \hat{J}(t)-\hat{J}(t-\Delta t) e^{-\lambda^{2} \Delta t}\right)\right] e^{-\lambda^{2} \Delta t}
$$

The predicted values for concentrations are then used to update the vorticity $\hat{\omega}(t+\Delta t)$ through Eq. D.7d and in turn to update $\hat{\psi}(t+\Delta t)$ through Eq. D.7f. Later, the predicted concentrations are corrected using Adam-Bashforth method:

$$
\hat{C}^{C}(t+\Delta t)=\left[\hat{C^{C}}(t)+\frac{\Delta t}{2}\left(\hat{J}(t)+\hat{J}^{p}(t+\Delta t) e^{\lambda^{2} \Delta t}\right)\right] e^{-\lambda^{2} \Delta t}
$$

With the updated concentrations, $\hat{\omega}(t+\Delta t)$ and $\hat{\psi}(t+\Delta t)$ are then corrected as well. This procedure is now iterated to reach the desired values of $\hat{C}_{i}(t+\Delta t), \hat{\omega}(t+\Delta t)$ and $\hat{\psi}(t+\Delta t)$. At the end, $C_{i}, \omega$ and $\psi$ is found in the real space using the inverse Hartley transform. 